\title{
IUCrJ
}

Volume 5 (2018)

Supporting information for article:

Bond orders for intermolecular interactions in crystals: Charge transfer, ionicity and the effect on intramolecular bonds

Khidhir Alhameedi, Amir Karton, Dylan Jayatilaka and Sajesh P. Thomas 


\title{
Supporting Information
}

\section{Bond orders for intermolecular interactions in crystals: Charge transfer, ionicity and the effect on intramolecular bonds}

\author{
Khidhir Alhameedi, ${ }^{* a b}$ Amir Karton, ${ }^{a}$ Dylan Jayatilaka, ${ }^{* a}$ and Sajesh P. Thomas ${ }^{* a}$ \\ ${ }^{a}$ School of Molecular Sciences, University of Western Australia, 35 Stirling Highway, Perth \\ 6009, Australia. \\ ${ }^{b}$ Department of Chemistry, College of Education for Pure Science, University of Karbala, \\ Karbala, Iraq. \\ E-mail: \\ sajeshpthomas@gmail.com \\ khidhir.alhameedi@research.uwa.edu.au
}

- Please note that the all output files are deposited for open access under figshare.com website, see the link: https://figshare.com/s/538fcbb3898fc764a21b. 


\section{Contents}

S1 Roby-Gould bond indices for hydrogen bonds

S2 Atom... atom ionic index versus molecule $\cdots$ molecule ionic index

S3 Hirshfeld atom charges $\quad 3$

S4 Interaction energies for hydrogen bonds, halogen bonds and chalcogen bonds

S5 Testing the conservation of Roby-Gould bond bond order

S6 The angle dependence of RGBI values

S7 Percentage of covalency

S8 Molecular structures of selected dimers 15

S8.1 Hydrogen bonds . . . . . . . . . . . . . . . . . . . . . . . . . . 16

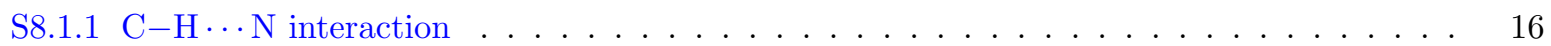

$\mathrm{S} 8.1 .2 \mathrm{~N}-\mathrm{H} \ldots \mathrm{N}$ interactions . . . . . . . . . . . . . . . . . . . . . . 21

$\mathrm{S} 8.1 .3 \mathrm{O}-\mathrm{H} \cdots \mathrm{N}$ interactions . . . . . . . . . . . . . . . . . . . . . 23

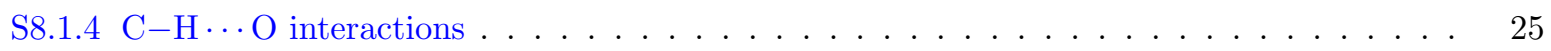

S8.1.5 $\mathrm{N}-\mathrm{H} \ldots \mathrm{O}$ interactions . . . . . . . . . . . . . . . . . . . . . 28

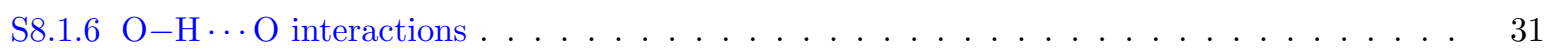

S8.2 Halogen bonds . . . . . . . . . . . . . . . . . . . . . . . . . . . 34

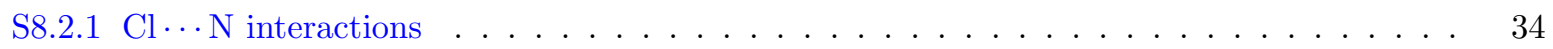

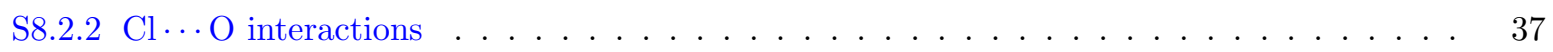

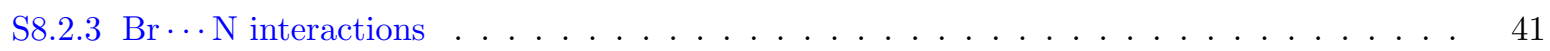

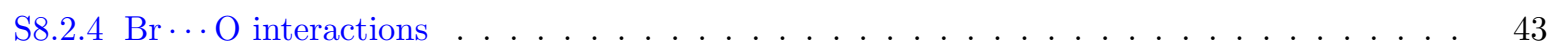

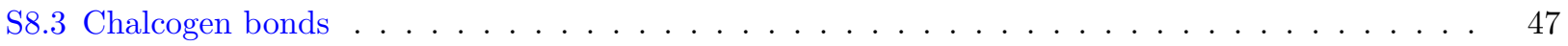

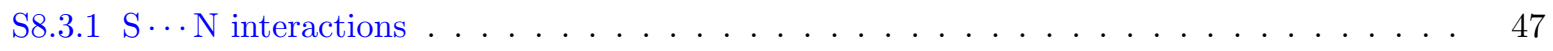

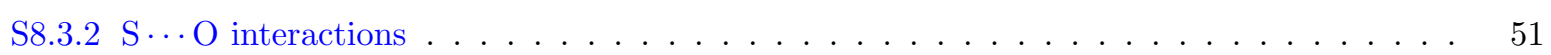

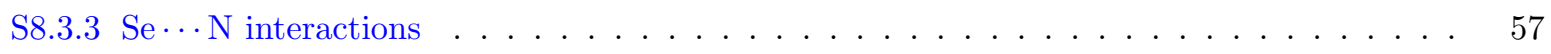

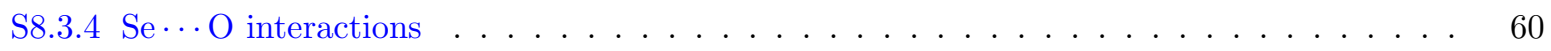




\section{S1 Roby-Gould bond indices for hydrogen bonds}

Table S1: Atom $\cdots$ atom and molecule $\cdots$ molecule Roby-Gould bond indices, covalent index $(c)$, ionic index $(i)$, and total bond index $(\tau)$, for hydrogen bonds $(\mathrm{D}-\mathrm{H} \cdots \mathrm{A}, \mathrm{D}=\mathrm{C}, \mathrm{N}, \mathrm{O}, \mathrm{A}=\mathrm{N}, \mathrm{O})$. The distances $d$ and the inter-penetration of van der Waals spheres $(\Delta d)$ are given in Angstrom $(\AA)$. The single and multipliable contact interactions in dimers are marked with superscript $s$ and $m$ ( and $m *$ for two identical interactions within one dimer).

\begin{tabular}{|c|c|c|c|c|c|c|c|c|c|c|}
\hline \multirow{2}{*}{$\begin{array}{l}\mathrm{D}-\mathrm{H} \cdots \mathrm{A} \\
\mathrm{CSD} \text { code }\end{array}$} & \multirow{2}{*}{$\begin{array}{c}d_{(\mathrm{H} \cdots \mathrm{A})} \\
/ \AA\end{array}$} & \multirow{2}{*}{$\begin{array}{c}\Delta d_{(\mathrm{H} \cdots \mathrm{A})} \\
/ \AA\end{array}$} & \multicolumn{4}{|c|}{ Atom-atom indices } & \multicolumn{4}{|c|}{ molecule-molecule indices } \\
\hline & & & $c$ & $i$ & $\tau$ & $\% c$ & $c$ & $i$ & $\tau$ & $\% c$ \\
\hline \multicolumn{11}{|l|}{$\mathrm{C}-\mathrm{H} \cdots \mathrm{N}$} \\
\hline $\mathrm{CYACHZO1}^{s}$ & 2.476 & 0.274 & 0.06 & 0.05 & 0.08 & 53.75 & 0.23 & 0.79 & 0.83 & 7.75 \\
\hline DMSDIM01 $^{s}$ & 2.522 & 0.228 & 0.05 & 0.08 & 0.10 & 23.19 & 0.16 & 0.06 & 0.17 & 87.53 \\
\hline MEADENO2 $^{s}$ & 2.524 & 0.226 & 0.06 & 0.15 & 0.16 & 12.49 & 0.19 & 0.52 & 0.56 & 11.75 \\
\hline TRAZOLO $^{s}$ & 2.346 & 0.404 & 0.07 & 0.13 & 0.15 & 19.80 & 0.22 & 1.12 & 1.14 & 3.79 \\
\hline BUGKIX01 $^{s}$ & 2.523 & 0.227 & 0.06 & 0.04 & 0.07 & 65.47 & 0.16 & 0.78 & 0.80 & 3.87 \\
\hline BUGKIX01 $^{s}$ & 2.321 & 0.429 & 0.07 & 0.20 & 0.21 & 10.56 & 0.28 & 0.44 & 0.52 & 28.21 \\
\hline $\mathrm{CYACHZ01}^{m}$ & 2.509 & 0.241 & 0.05 & 0.11 & 0.12 & 15.14 & 0.17 & 0.72 & 0.74 & 5.21 \\
\hline NURWOM02 $2^{m *}$ & 2.495 & 0.255 & 0.05 & 0.14 & 0.15 & 9.63 & 0.33 & 0.00 & 0.33 & 100.00 \\
\hline NURWOM02 $2^{m}$ & 2.456 & 0.294 & 0.07 & 0.16 & 0.17 & 14.76 & 0.33 & 0.12 & 0.35 & 89.18 \\
\hline TEPNIT0 $^{m *}$ & 2.503 & 0.247 & 0.05 & 0.11 & 0.12 & 16.18 & 0.29 & 0.00 & 0.29 & 100.00 \\
\hline${\mathrm{XEHMOM} 01^{m}}^{m}$ & 2.513 & 0.237 & 0.05 & 0.04 & 0.07 & 62.01 & 0.37 & 0.87 & 0.94 & 15.53 \\
\hline${\mathrm{XEHMOM} 01^{m}}^{m}$ & 2.492 & 0.258 & 0.05 & 0.01 & 0.05 & 97.42 & 0.22 & 0.94 & 0.97 & 5.35 \\
\hline${\mathrm{XEHMOM} 01^{m}}^{m}$ & 2.707 & 0.043 & 0.04 & -0.03 & 0.05 & 69.86 & & & & \\
\hline \multicolumn{11}{|l|}{$\mathrm{N}-\mathrm{H} \cdots \mathrm{N}$} \\
\hline BAZGOY0 $5^{s}$ & 2.363 & 0.387 & 0.09 & 0.22 & 0.23 & 14.35 & 0.23 & 0.49 & 0.54 & 18.52 \\
\hline DMSDIM01 $^{s}$ & 2.360 & 0.390 & 0.09 & 0.20 & 0.22 & 17.75 & 0.34 & 0.25 & 0.42 & 64.31 \\
\hline FORAMO01 $^{s}$ & 2.096 & 0.654 & 0.12 & 0.20 & 0.23 & 25.80 & 0.35 & 0.25 & 0.43 & 65.65 \\
\hline IMAZOL06 $^{s}$ & 1.808 & 0.942 & 0.18 & 0.23 & 0.29 & 38.04 & 0.50 & 0.23 & 0.55 & 82.46 \\
\hline TRAZOL02 $^{s}$ & 1.788 & 0.962 & 0.18 & 0.23 & 0.29 & 39.18 & 0.51 & 0.31 & 0.60 & 72.60 \\
\hline \multicolumn{11}{|l|}{$\mathrm{O}-\mathrm{H} \cdots \mathrm{N}$} \\
\hline FORAMO01 $^{s}$ & 1.809 & 0.941 & 0.20 & 0.26 & 0.33 & 37.44 & 0.50 & 0.04 & 0.50 & 99.26 \\
\hline GLOXIM11 $^{s}$ & 1.805 & 0.945 & 0.20 & 0.30 & 0.36 & 31.56 & 0.53 & -0.29 & 0.61 & 76.44 \\
\hline MAMPOL02 $^{s}$ & 1.758 & 0.992 & 0.22 & 0.28 & 0.36 & 38.18 & 0.49 & 0.53 & 0.72 & 46.52 \\
\hline LOLSUA $^{m}$ & 1.535 & 1.215 & 0.31 & 0.28 & 0.41 & 55.51 & 0.77 & -1.19 & 1.42 & 29.70 \\
\hline ULAWAF0 $5^{m}$ & 1.398 & 1.352 & 0.38 & 0.29 & 0.48 & 63.64 & 0.86 & -0.51 & 1.00 & 73.94 \\
\hline \multicolumn{11}{|l|}{$\mathrm{C}-\mathrm{H} \cdots \mathrm{O}$} \\
\hline${\mathrm{ABINOR} 04^{s}}^{\mathrm{S}}$ & 2.519 & 0.201 & 0.05 & 0.18 & 0.18 & 6.41 & 0.23 & 0.56 & 0.60 & 13.95 \\
\hline NBONAN01 $^{s}$ & 2.298 & 0.422 & 0.05 & 0.15 & 0.16 & 11.10 & 0.20 & 1.08 & 1.10 & 3.40 \\
\hline POKKAD01 $^{s}$ & 2.497 & 0.223 & 0.03 & 0.15 & 0.15 & 4.88 & 0.11 & 0.33 & 0.35 & 9.25 \\
\hline $\mathrm{CYACHZO1}^{m *}$ & 2.412 & 0.308 & 0.05 & 0.14 & 0.15 & 11.93 & 0.35 & 0.00 & 0.35 & 100.00 \\
\hline FACETA $01^{m *}$ & 2.447 & 0.273 & 0.06 & 0.14 & 0.15 & 15.61 & 0.26 & 0.00 & 0.26 & 100.00 \\
\hline GLYGLY0 $^{m}$ & 2.521 & 0.199 & 0.06 & 0.14 & 0.15 & 14.98 & 0.41 & 0.37 & 0.55 & 55.32 \\
\hline GLYGLY0 $^{m}$ & 2.301 & 0.419 & 0.07 & 0.13 & 0.15 & 22.48 & & & & \\
\hline METURA01 $^{m}$ & 2.322 & 0.398 & 0.06 & 0.16 & 0.17 & 13.24 & 0.26 & 0.62 & 0.67 & 15.35 \\
\hline \multicolumn{11}{|l|}{$\mathrm{N}-\mathrm{H} \cdots \mathrm{O}$} \\
\hline BZAMID02 $2^{m *}$ & 1.910 & 0.810 & 0.14 & 0.24 & 0.28 & 25.60 & 0.71 & 0.00 & 0.71 & 100.00 \\
\hline CYURAC $12^{m *}$ & 1.774 & 0.946 & 0.16 & 0.25 & 0.29 & 28.76 & 0.83 & 0.00 & 0.83 & 100.00 \\
\hline FACETA01 ${ }^{m *}$ & 1.909 & 0.811 & 0.14 & 0.24 & 0.28 & 24.68 & 0.71 & 0.00 & 0.71 & 100.00 \\
\hline ULAWAF $05^{m *}$ & 1.894 & 0.826 & 0.14 & 0.24 & 0.28 & 25.18 & 0.72 & 0.00 & 0.72 & 100.00 \\
\hline UROXAL01 ${ }^{m *}$ & 1.924 & 0.796 & 0.14 & 0.24 & 0.28 & 25.50 & 0.71 & 0.00 & 0.71 & 100.00 \\
\hline ZECWUB01 ${ }^{m *}$ & 1.882 & 0.838 & 0.14 & 0.24 & 0.28 & 25.49 & 0.72 & 0.00 & 0.72 & 100.00 \\
\hline \multicolumn{11}{|l|}{$\mathrm{O}-\mathrm{H} \cdots \mathrm{O}$} \\
\hline BESKAL10 ${ }^{m *}$ & 1.715 & 1.005 & 0.20 & 0.31 & 0.37 & 28.64 & 0.88 & 0.00 & 0.88 & 100.00 \\
\hline CUKCAM18 ${ }^{m *}$ & 1.630 & 1.090 & 0.23 & 0.31 & 0.38 & 36.20 & 0.97 & 0.00 & 0.97 & 100.00 \\
\hline SALIAC $12^{m *}$ & 1.651 & 1.069 & 0.21 & 0.30 & 0.37 & 33.59 & 0.95 & 0.00 & 0.95 & 100.00 \\
\hline $\mathrm{SUCACB} 3^{m *}$ & 1.670 & 1.050 & 0.22 & 0.31 & 0.38 & 33.72 & 0.93 & 0.00 & 0.93 & 100.00 \\
\hline ZZZEEU05 ${ }^{m *}$ & 1.653 & 1.067 & 0.22 & 0.30 & 0.38 & 34.80 & 0.94 & 0.00 & 0.94 & 100.00 \\
\hline
\end{tabular}


S2 Atom ... atom ionic index versus molecule $\cdots$ molecule ionic index

Table S2: Summarize table of Roby-Gould bond indices, covalent index $(c)$, ionic index $(i)$, and total bond index $(\tau)$, for selected 35 examples, where their ionic indices are higher in atom $\cdots$ atom interaction comparing those in molecule $\cdots$ molecule interactions.

\begin{tabular}{|c|c|c|c|c|c|c|c|c|c|c|}
\hline \multirow{2}{*}{$\begin{array}{l}\mathrm{D}-\mathrm{H} \cdots \mathrm{A} \\
\mathrm{CSD} \text { code }\end{array}$} & \multirow{2}{*}{$\begin{array}{c}d_{(\mathrm{H} \ldots \mathrm{A})} \\
/ \AA \AA\end{array}$} & \multirow{2}{*}{$\begin{array}{c}\Delta d_{(\mathrm{H} \cdots \mathrm{A})} \\
/ \AA\end{array}$} & \multicolumn{4}{|c|}{ Atom-atom indices } & \multicolumn{4}{|c|}{ molecule-molecule indices } \\
\hline & & & $c$ & $i$ & $\tau$ & $\% c$ & $c$ & $i$ & $\tau$ & $\% c$ \\
\hline \multicolumn{11}{|l|}{$\mathrm{C}-\mathrm{H} \cdots \mathrm{N}$} \\
\hline DMSDIM01 $^{s}$ & 2.522 & 0.228 & 0.05 & 0.08 & 0.10 & 23.19 & 0.16 & 0.06 & 0.17 & 87.53 \\
\hline NURWOM02 $^{m *}$ & 2.495 & 0.255 & 0.05 & 0.14 & 0.15 & 9.63 & 0.33 & 0.00 & 0.33 & 100.00 \\
\hline NURWOM02 $^{m}$ & 2.456 & 0.294 & 0.07 & 0.16 & 0.17 & 14.76 & 0.33 & 0.12 & 0.35 & 89.18 \\
\hline TEPNIT0 $4^{m *}$ & 2.503 & 0.247 & 0.05 & 0.11 & 0.12 & 16.18 & 0.29 & 0.00 & 0.29 & 100.00 \\
\hline \multicolumn{11}{|l|}{$\mathrm{O}-\mathrm{H} \cdots \mathrm{N}$} \\
\hline FORAMO01 $^{s}$ & 1.809 & 0.941 & 0.20 & 0.26 & 0.33 & 37.44 & 0.50 & 0.04 & 0.50 & 99.26 \\
\hline \multicolumn{11}{|l|}{$\mathrm{C}-\mathrm{H} \cdots \mathrm{O}$} \\
\hline $\mathrm{CYACHZO1}^{m *}$ & 2.412 & 0.308 & 0.05 & 0.14 & 0.15 & 11.93 & 0.35 & 0.00 & 0.35 & 100.00 \\
\hline FACETA01 $1^{m *}$ & 2.447 & 0.273 & 0.06 & 0.14 & 0.15 & 15.61 & 0.26 & 0.00 & 0.26 & 100.00 \\
\hline \multicolumn{11}{|l|}{$\mathrm{N}-\mathrm{H} \cdots \mathrm{O}$} \\
\hline BZAMID02 $2^{m *}$ & 1.910 & 0.810 & 0.14 & 0.24 & 0.28 & 25.60 & 0.71 & 0.00 & 0.71 & 100.00 \\
\hline CYURAC12 $2^{m *}$ & 1.774 & 0.946 & 0.16 & 0.25 & 0.29 & 28.76 & 0.83 & 0.00 & 0.83 & 100.00 \\
\hline FACETA01 $1^{m *}$ & 1.909 & 0.811 & 0.14 & 0.24 & 0.28 & 24.68 & 0.71 & 0.00 & 0.71 & 100.00 \\
\hline ULAWAF $05^{m *}$ & 1.894 & 0.826 & 0.14 & 0.24 & 0.28 & 25.18 & 0.72 & 0.00 & 0.72 & 100.00 \\
\hline UROXAL01 $^{m *}$ & 1.924 & 0.796 & 0.14 & 0.24 & 0.28 & 25.50 & 0.71 & 0.00 & 0.71 & 100.00 \\
\hline ZECWUB01 ${ }^{m *}$ & 1.882 & 0.838 & 0.14 & 0.24 & 0.28 & 25.49 & 0.72 & 0.00 & 0.72 & 100.00 \\
\hline \multicolumn{11}{|l|}{$\mathrm{O}-\mathrm{H} \cdots \mathrm{O}$} \\
\hline BESKAL10 ${ }^{m *}$ & 1.715 & 1.005 & 0.20 & 0.31 & 0.37 & 28.64 & 0.88 & 0.00 & 0.88 & 100.00 \\
\hline CUKCAM $18^{m *}$ & 1.630 & 1.090 & 0.23 & 0.31 & 0.38 & 36.20 & 0.97 & 0.00 & 0.97 & 100.00 \\
\hline SALIAC $12^{m *}$ & 1.651 & 1.069 & 0.21 & 0.30 & 0.37 & 33.59 & 0.95 & 0.00 & 0.95 & 100.00 \\
\hline SUCACB03 ${ }^{m *}$ & 1.670 & 1.050 & 0.22 & 0.31 & 0.38 & 33.72 & 0.93 & 0.00 & 0.93 & 100.00 \\
\hline ZZZEEU0 $^{m *}$ & 1.653 & 1.067 & 0.22 & 0.30 & 0.38 & 34.80 & 0.94 & 0.00 & 0.94 & 100.00 \\
\hline \multicolumn{11}{|l|}{$\mathrm{Cl} \cdots \mathrm{N}$} \\
\hline PCLPYR $^{s}$ & 3.014 & 0.286 & 0.06 & 0.27 & 0.28 & 4.87 & 0.14 & -0.02 & 0.14 & 97.39 \\
\hline BZQDCL11 ${ }^{s}$ & 3.056 & 0.214 & 0.03 & -0.08 & 0.08 & 10.90 & 0.09 & 0.06 & 0.11 & 70.69 \\
\hline JOJTIL $^{s}$ & 2.948 & 0.322 & 0.06 & -0.16 & 0.17 & 10.76 & 0.11 & -0.01 & 0.11 & 99.37 \\
\hline GEXWUB $^{s}$ & 3.002 & 0.268 & 0.03 & -0.10 & 0.10 & 7.47 & 0.08 & 0.07 & 0.11 & 52.07 \\
\hline \multicolumn{11}{|l|}{$\mathrm{Br} \cdots \mathrm{N}$} \\
\hline BONFIT $^{s}$ & 2.863 & 0.537 & 0.13 & 0.25 & 0.28 & 22.17 & 0.21 & -0.05 & 0.21 & 95.10 \\
\hline QONHUX $^{s}$ & 3.093 & 0.307 & 0.07 & 0.24 & 0.25 & 9.23 & 0.16 & -0.07 & 0.18 & 82.95 \\
\hline $\mathrm{RIRFOO}^{s}$ & 3.164 & 0.236 & 0.06 & 0.26 & 0.26 & 5.61 & 0.14 & -0.22 & 0.26 & 27.91 \\
\hline \multicolumn{11}{|l|}{$\mathrm{Br} \cdots \mathrm{O}$} \\
\hline BMLTAA $^{s}$ & 3.082 & 0.288 & 0.04 & -0.11 & 0.12 & 10.36 & 0.11 & 0.00 & 0.11 & 99.81 \\
\hline $\mathrm{CIRSON}^{s}$ & 3.149 & 0.221 & 0.03 & -0.12 & 0.13 & 5.18 & 0.12 & 0.09 & 0.15 & 62.28 \\
\hline VAQXUG $^{s}$ & 3.160 & 0.210 & 0.03 & -0.10 & 0.10 & 10.75 & 0.12 & -0.04 & 0.13 & 92.26 \\
\hline VEWTAU $^{s}$ & 2.893 & 0.477 & 0.06 & 0.07 & 0.10 & 42.44 & 0.14 & 0.00 & 0.14 & 99.99 \\
\hline \multicolumn{11}{|l|}{$\mathrm{S} \cdots \mathrm{O}$} \\
\hline ADOFEF $^{m *}$ & 3.101 & 0.219 & 0.05 & -0.14 & 0.15 & 10.52 & 0.25 & 0.00 & 0.25 & 100.00 \\
\hline ZAVHEJ $^{m *}$ & 2.924 & 0.396 & 0.08 & -0.18 & 0.20 & 16.93 & 0.25 & 0.00 & 0.25 & 100.00 \\
\hline \multicolumn{11}{|l|}{$\mathrm{Se} \cdots \mathrm{N}$} \\
\hline $\mathrm{NECZUQ}^{m *}$ & 2.877 & 0.573 & 0.18 & -0.15 & 0.23 & 56.55 & 0.71 & 0.00 & 0.71 & 100.00 \\
\hline \multicolumn{11}{|l|}{$\mathrm{Se} \cdots \mathrm{O}$} \\
\hline BOJCOS $^{m *}$ & 3.042 & 0.378 & 0.08 & -0.21 & 0.23 & 13.69 & 0.27 & 0.00 & 0.27 & 100.00 \\
\hline LEDGAD $^{m}$ & 3.188 & 0.232 & 0.05 & -0.09 & 0.11 & 22.64 & 0.22 & 0.05 & 0.23 & 94.68 \\
\hline
\end{tabular}




\section{S3 Hirshfeld atom charges}

In this section we present the values of Hirshfeld atom charges in atomic unit (a.u.), which represents the partial charges of atoms in monomers and dimers. Charges are given for atoms X and A involved in in the hydrogen bonds (Table S3), halogen bonds (Table S4), and chalcogen bonds (Table S5). The difference in Hirshfeld charges for atoms $\mathrm{X}$ and $\mathrm{A}$ from monomer upon dimer formation are given as $\Delta q$. 
Table S3: Hirshfeld charges of atoms involved in hydrogen bonds and the charge transfer due to intermolecular interactions.

\begin{tabular}{|c|c|c|c|c|c|c|c|}
\hline $\begin{array}{l}\mathrm{D}-\mathrm{H} \cdots \mathrm{A} \\
\mathrm{CSD} \text { code }\end{array}$ & $\mathrm{A} \cdots \mathrm{H}$ & $\begin{array}{c}q_{A}^{\text {dimer }} \\
\text { /a.u. }\end{array}$ & $\begin{array}{c}q_{H}^{\text {dimer }} \\
\text { /a.u. }\end{array}$ & $\begin{array}{c}q_{A}^{\text {monomer }} \\
/ \text { a.u. }\end{array}$ & $\begin{array}{c}q_{H}^{\text {monomer }} \\
\text { /a.u. }\end{array}$ & $\begin{array}{l}\Delta q_{A} \\
\text { /a.u. }\end{array}$ & $\begin{array}{l}\Delta q_{H} \\
\text { /a.u. }\end{array}$ \\
\hline \multicolumn{8}{|l|}{$\mathrm{C}-\mathrm{H} \cdots \mathrm{N}$} \\
\hline CYACHZ01 $^{s}$ & N6-H4 & -0.185 & 0.061 & -0.205 & 0.072 & 0.020 & -0.011 \\
\hline DMSDIM01s $^{s}$ & N3-H3 & -0.343 & 0.043 & -0.357 & 0.054 & 0.014 & -0.011 \\
\hline MEADEN02 ${ }^{s}$ & N7-H5 & -0.181 & 0.030 & -0.201 & 0.050 & 0.021 & -0.020 \\
\hline TRAZOLO2 $^{s}$ & N5-H3 & -0.132 & 0.046 & -0.162 & 0.072 & 0.030 & -0.026 \\
\hline BUGKIX01s & N6-H1 & -0.136 & 0.080 & -0.150 & 0.099 & 0.015 & -0.019 \\
\hline BUGKIX01s & N5-H1 & -0.134 & 0.078 & -0.150 & 0.099 & 0.016 & -0.021 \\
\hline $\mathrm{CYACHZO1}^{m}$ & N6-H5 & -0.195 & 0.051 & -0.205 & 0.063 & 0.009 & -0.012 \\
\hline NURWOM02 $2^{m *}$ & N1-H10 & -0.126 & 0.035 & -0.137 & 0.046 & 0.012 & -0.011 \\
\hline NURWOM02 ${ }^{m}$ & $\mathrm{~N} 4-\mathrm{H} 1$ & -0.119 & 0.030 & -0.139 & 0.048 & 0.021 & -0.018 \\
\hline TEPNIT0 $4^{m *}$ & N1-H5 & -0.191 & 0.054 & -0.195 & 0.066 & 0.005 & -0.012 \\
\hline XEHMOM01 $^{m}$ & N5-H1 & -0.177 & 0.055 & -0.198 & 0.068 & 0.021 & -0.013 \\
\hline XEHMOM01 ${ }^{m}$ & N5-H9 & -0.184 & 0.034 & -0.198 & 0.048 & 0.014 & -0.013 \\
\hline XEHMOM01 ${ }^{m}$ & $\mathrm{~N} 6-\mathrm{H} 8$ & -0.186 & 0.033 & -0.195 & 0.046 & 0.010 & -0.013 \\
\hline \multicolumn{8}{|l|}{$\mathrm{N}-\mathrm{H} \cdots \mathrm{N}$} \\
\hline BAZGOY05 ${ }^{s}$ & $\mathrm{~N} 2-\mathrm{H} 2$ & -0.143 & 0.084 & -0.165 & 0.112 & 0.022 & -0.029 \\
\hline${\text { DMSDIM } 01^{s}}$ & N3-H1 & -0.320 & 0.061 & -0.357 & 0.084 & 0.038 & -0.023 \\
\hline${\text { FORAMO } 01^{s}}$ & $\mathrm{~N} 4-\mathrm{H} 2$ & -0.122 & 0.078 & -0.167 & 0.122 & 0.044 & -0.045 \\
\hline IMAZOL06 $^{s}$ & N4-H1 & -0.133 & 0.086 & -0.206 & 0.156 & 0.073 & -0.070 \\
\hline TRAZOLO2 $^{s}$ & $\mathrm{~N} 6-\mathrm{H} 1$ & -0.137 & 0.089 & -0.208 & 0.165 & 0.071 & -0.076 \\
\hline \multicolumn{8}{|l|}{$\mathrm{O}-\mathrm{H} \cdots \mathrm{N}$} \\
\hline FORAMO01 $^{s}$ & $\mathrm{~N} 4-\mathrm{H} 4$ & -0.098 & 0.084 & -0.167 & 0.164 & 0.068 & -0.080 \\
\hline GLOXIM11 $^{s}$ & $\mathrm{~N} 3-\mathrm{H} 2$ & -0.014 & 0.092 & -0.081 & 0.176 & 0.067 & -0.085 \\
\hline MAMPOL02 $^{s}$ & $\mathrm{~N} 2-\mathrm{H} 5$ & -0.101 & 0.095 & -0.173 & 0.177 & 0.072 & -0.082 \\
\hline LOLSUA $^{m}$ & $\mathrm{~N}-\mathrm{H} 1$ & -0.060 & 0.093 & -0.157 & 0.045 & 0.097 & 0.048 \\
\hline ULAWAF $05^{m}$ & $\mathrm{~N} 1-\mathrm{H} 1$ & -0.026 & 0.103 & -0.150 & 0.237 & 0.124 & -0.135 \\
\hline \multicolumn{8}{|l|}{$\mathrm{C}-\mathrm{H} \cdots \mathrm{O}$} \\
\hline ABINOR04 ${ }^{s}$ & $\mathrm{O} 7-\mathrm{H} 6$ & -0.204 & 0.031 & -0.223 & 0.046 & 0.018 & -0.016 \\
\hline NBONAN01 $^{s}$ & O6-H3 & -0.219 & 0.045 & -0.243 & 0.056 & 0.024 & -0.011 \\
\hline POKKAD01 ${ }^{s}$ & $\mathrm{O} 5-\mathrm{H} 3$ & -0.278 & 0.046 & -0.295 & 0.052 & 0.017 & -0.005 \\
\hline CYACHZ01 ${ }^{m *}$ & $\mathrm{O} 1-\mathrm{H} 9$ & -0.286 & 0.068 & -0.290 & 0.072 & 0.004 & -0.003 \\
\hline FACETA01 $1^{m *}$ & $\mathrm{O} 2-\mathrm{H} 2$ & -0.325 & 0.048 & -0.335 & 0.061 & 0.010 & -0.012 \\
\hline GLYGLY0 $^{m}$ & $\mathrm{O} 6-\mathrm{H} 7$ & -0.376 & 0.046 & -0.422 & 0.042 & 0.046 & 0.005 \\
\hline GLYGLY0 $^{m}$ & O4-H4 & -0.315 & 0.048 & -0.323 & 0.076 & 0.008 & -0.028 \\
\hline METURA01 $^{m}$ & $\mathrm{O} 4-\mathrm{H} 4$ & -0.301 & 0.034 & -0.319 & 0.049 & 0.019 & -0.016 \\
\hline \multicolumn{8}{|l|}{$\mathrm{N}-\mathrm{H} \cdots \mathrm{O}$} \\
\hline BZAMID02 $2^{m *}$ & $\mathrm{O} 2-\mathrm{H} 6$ & -0.290 & 0.096 & -0.321 & 0.138 & 0.031 & -0.042 \\
\hline CYURAC12 $2^{m *}$ & $\mathrm{O} 5-\mathrm{H} 2$ & -0.258 & 0.111 & -0.305 & 0.171 & 0.047 & -0.060 \\
\hline FACETA $01^{m *}$ & $\mathrm{O} 1-\mathrm{H} 7$ & -0.303 & 0.100 & -0.335 & 0.144 & 0.031 & -0.043 \\
\hline ULAWAF0 $05^{m *}$ & O1-H11 & -0.282 & 0.099 & -0.313 & 0.143 & 0.031 & -0.044 \\
\hline UROXAL01 $^{m *}$ & $\mathrm{O} 2-\mathrm{H} 4$ & -0.356 & 0.097 & -0.394 & 0.142 & 0.038 & -0.045 \\
\hline ZECWUB01 ${ }^{m *}$ & O1-H3 & -0.270 & 0.103 & -0.283 & 0.152 & 0.013 & -0.049 \\
\hline \multicolumn{8}{|l|}{$\mathrm{O}-\mathrm{H} \cdots \mathrm{O}$} \\
\hline BESKAL10 ${ }^{m *}$ & O3-H11 & -0.213 & 0.133 & -0.261 & 0.199 & 0.047 & -0.067 \\
\hline CUKCAM $18^{m *}$ & O8-H4 & -0.225 & 0.129 & -0.278 & 0.211 & 0.054 & -0.083 \\
\hline SALIAC $12^{m *}$ & $\mathrm{O} 2-\mathrm{H} 7$ & -0.212 & 0.123 & -0.269 & 0.200 & 0.057 & -0.077 \\
\hline $\mathrm{SUCACB} 3^{m *}$ & O1-H9 & -0.237 & 0.122 & -0.295 & 0.195 & 0.057 & -0.072 \\
\hline ZZZEEU05 ${ }^{m *}$ & O7-H4 & -0.220 & 0.121 & -0.278 & 0.200 & 0.059 & -0.079 \\
\hline
\end{tabular}


Table S4: Hirshfeld charges of atoms involved in halogen bonds and the charge transfer due to intermolecular interactions.

\begin{tabular}{|c|c|c|c|c|c|c|c|}
\hline $\begin{array}{l}\mathrm{X} \cdots \mathrm{A} \\
\text { CSD code }\end{array}$ & $\mathrm{X} \cdots \mathrm{A}$ & $\begin{array}{c}q_{A}^{\text {dimer }} \\
\text { /a.u. }\end{array}$ & $\begin{array}{c}q_{H}^{\text {dimer }} \\
\quad / \text { a.u. }\end{array}$ & $\begin{array}{c}q_{A}^{\text {monomer }} \\
\quad / \text { a.u. }\end{array}$ & $\begin{array}{c}q_{H}^{\text {monomer }} \\
\quad / \text { a.u. }\end{array}$ & $\begin{array}{l}\Delta q_{A} \\
/ \text { a.u. }\end{array}$ & $\begin{array}{l}\Delta q_{H} \\
/ \text { a.u. }\end{array}$ \\
\hline \multicolumn{8}{|l|}{$\mathrm{Cl} \cdots \mathrm{N}$} \\
\hline $\mathrm{CCACENN}^{s}$ & $\mathrm{Cl} 1-\mathrm{N} 2$ & 0.086 & -0.161 & 0.097 & -0.171 & -0.011 & 0.010 \\
\hline DESKER01 $^{s}$ & $\mathrm{Cl1}-\mathrm{N} 4$ & -0.021 & -0.066 & -0.009 & -0.082 & -0.012 & 0.016 \\
\hline NABZAS $^{s}$ & $\mathrm{Cl} 1-\mathrm{N} 2$ & 0.005 & -0.164 & 0.019 & -0.169 & -0.014 & 0.006 \\
\hline PCLPYR $^{s}$ & $\mathrm{Cl} 3-\mathrm{N} 2$ & -0.008 & -0.122 & 0.002 & -0.133 & -0.011 & 0.011 \\
\hline VUGSIZ $^{s}$ & $\mathrm{Cl1}-\mathrm{N} 5$ & -0.020 & -0.139 & -0.007 & -0.150 & -0.013 & 0.010 \\
\hline PALPAV $^{m}$ & $\mathrm{Cl1}-\mathrm{N} 7$ & 0.000 & -0.152 & -0.024 & -0.159 & 0.024 & 0.007 \\
\hline $\mathrm{XIZPON}^{s}$ & Cl1-N3 & 0.014 & -0.115 & 0.012 & -0.120 & 0.002 & 0.005 \\
\hline \multicolumn{8}{|l|}{$\mathrm{Cl} \cdots \mathrm{O}$} \\
\hline $\mathrm{BEDMON}^{s}$ & $\mathrm{Cl1}-\mathrm{O} 6$ & 0.008 & -0.241 & 0.010 & -0.243 & -0.002 & 0.002 \\
\hline BZQDCL11 $^{s}$ & $\mathrm{Cl1}-\mathrm{O} 3$ & -0.011 & -0.203 & -0.009 & -0.210 & -0.002 & 0.007 \\
\hline CORDUI $^{s}$ & $\mathrm{Cl1}-\mathrm{O} 4$ & -0.054 & -0.306 & -0.056 & -0.316 & 0.002 & 0.010 \\
\hline DCLBZQ20 $^{s}$ & $\mathrm{Cl1}-\mathrm{O} 4$ & -0.007 & -0.201 & -0.005 & -0.210 & -0.002 & 0.008 \\
\hline IRUFEH01 $^{s}$ & $\mathrm{Cl1}-\mathrm{O} 4$ & 0.006 & -0.231 & 0.015 & -0.240 & -0.009 & 0.010 \\
\hline JOJTIL $^{s}$ & $\mathrm{Cl1}-\mathrm{O} 3$ & 0.033 & -0.210 & 0.044 & -0.216 & -0.011 & 0.006 \\
\hline RUBSUD $^{s}$ & $\mathrm{Cl1}-\mathrm{O} 9$ & 0.038 & -0.153 & 0.057 & -0.157 & -0.019 & 0.004 \\
\hline${\text { TCACAD } 01^{s}}^{s}$ & $\mathrm{Cl} 2-\mathrm{O} 4$ & -0.040 & -0.249 & -0.039 & -0.257 & 0.000 & 0.007 \\
\hline GEXWUB $^{s}$ & $\mathrm{Cl} 2-\mathrm{O} 4$ & 0.003 & -0.242 & -0.002 & -0.247 & 0.005 & 0.005 \\
\hline PEPFUL $^{s}$ & $\mathrm{Cl} 2-\mathrm{O} 1$ & 0.002 & -0.220 & 0.001 & -0.223 & 0.001 & 0.003 \\
\hline \multicolumn{8}{|l|}{$\mathrm{Br} \cdots \mathrm{N}$} \\
\hline $\mathrm{BCACENN}^{s}$ & $\mathrm{Br} 1-\mathrm{N} 2$ & 0.107 & -0.155 & 0.129 & -0.173 & -0.021 & 0.018 \\
\hline BONFIT $^{s}$ & Br2-N3 & 0.008 & -0.107 & 0.035 & -0.126 & -0.027 & 0.019 \\
\hline $\mathrm{QONHUX}^{s}$ & Br2-N3 & -0.001 & -0.143 & 0.002 & -0.153 & -0.003 & 0.010 \\
\hline $\mathrm{RIRFOON}^{s}$ & $\mathrm{Br} 1-\mathrm{N} 4$ & -0.034 & -0.128 & -0.024 & -0.145 & -0.010 & 0.017 \\
\hline $\mathrm{KUYCUD}^{s}$ & $\mathrm{Br} 1-\mathrm{N} 7$ & 0.028 & -0.128 & 0.047 & -0.151 & -0.019 & 0.023 \\
\hline \multicolumn{8}{|l|}{$\mathrm{Br} \cdots \mathrm{O}$} \\
\hline BMLTAA $^{s}$ & Br1-O3 & 0.034 & -0.198 & 0.040 & -0.204 & -0.006 & 0.006 \\
\hline CIRSONN $^{s}$ & Br1-O4 & -0.037 & -0.125 & -0.027 & -0.135 & -0.010 & 0.009 \\
\hline $\mathrm{JEVVOW}^{s}$ & $\mathrm{Br} 1-\mathrm{O} 2$ & -0.037 & -0.302 & -0.031 & -0.322 & -0.006 & 0.020 \\
\hline $\mathrm{VAQXUG}^{s}$ & $\mathrm{Br} 4-\mathrm{O} 2$ & 0.058 & -0.271 & 0.053 & -0.277 & 0.005 & 0.006 \\
\hline VEWTAU ${ }^{s}$ & $\mathrm{Br} 2-\mathrm{O} 4$ & -0.023 & -0.275 & -0.027 & -0.269 & 0.003 & -0.006 \\
\hline VEWTEY ${ }^{s}$ & Br1-O3 & -0.025 & -0.071 & -0.027 & -0.076 & 0.001 & 0.005 \\
\hline VITVEZ $^{s}$ & $\mathrm{Br} 2-\mathrm{O} 6$ & 0.015 & -0.176 & 0.024 & -0.179 & -0.010 & 0.003 \\
\hline WADFIR $^{s}$ & $\mathrm{Br} 1-\mathrm{O} 4$ & -0.028 & -0.249 & -0.023 & -0.261 & -0.005 & 0.012 \\
\hline $\operatorname{ACETBR} 02^{s}$ & $\mathrm{Br} 1-\mathrm{O}$ & -0.002 & -0.229 & 0.000 & -0.265 & -0.002 & 0.035 \\
\hline
\end{tabular}


Table S5: Hirshfeld charges of atoms involved in chalcogen bonds and the charge transfer due to intermolecular interactions.

\begin{tabular}{|c|c|c|c|c|c|c|c|}
\hline $\begin{array}{l}\text { Y } \cdots A \\
\text { CSD code }\end{array}$ & $\mathrm{Y} \cdots \mathrm{A}$ & $\begin{array}{c}q_{A}^{\text {dimer }} \\
\text { /a.u. }\end{array}$ & $\begin{array}{c}q_{H}^{\text {dimer }} \\
\text { /a.u. }\end{array}$ & $\begin{array}{c}q_{A}^{\text {monomer }} \\
\quad / \text { a.u. }\end{array}$ & $\begin{array}{l}q_{H}^{\text {monomer }} \\
\quad / \text { a.u. }\end{array}$ & $\begin{array}{l}\Delta q_{A} \\
\text { /a.u. }\end{array}$ & $\begin{array}{l}\Delta q_{H} \\
\text { /a.u. }\end{array}$ \\
\hline \multicolumn{8}{|l|}{$\mathrm{S} \cdots \mathrm{N}$} \\
\hline CEBYUD $^{s}$ & $\mathrm{~S} 1-\mathrm{N} 8$ & 0.224 & -0.145 & 0.231 & -0.149 & -0.007 & 0.004 \\
\hline QOBFUI $^{s}$ & S1-N5 & 0.144 & 0.004 & 0.158 & -0.014 & -0.014 & 0.019 \\
\hline SAZCEC $^{s}$ & $\mathrm{~S} 1-\mathrm{N} 4$ & 0.097 & -0.090 & 0.112 & -0.105 & -0.016 & 0.016 \\
\hline GEDHAY $^{m}$ & $\mathrm{~S} 2-\mathrm{N} 8$ & 0.081 & -0.228 & 0.099 & -0.259 & -0.018 & 0.030 \\
\hline GEDHAY $^{m}$ & $\mathrm{~S} 1-\mathrm{N} 8$ & 0.282 & -0.228 & 0.291 & -0.259 & -0.009 & 0.030 \\
\hline IFULUQ04 $^{m}$ & $\mathrm{~S} 1-\mathrm{N} 4$ & 0.147 & -0.184 & 0.120 & -0.172 & 0.027 & -0.012 \\
\hline WASHEE $^{m}$ & S3-N1 & 0.174 & -0.184 & 0.131 & -0.171 & 0.043 & -0.014 \\
\hline WASHEE $^{m}$ & S4-N1 & 0.149 & -0.184 & 0.120 & -0.171 & 0.028 & -0.014 \\
\hline WUXPAG $^{m}$ & S2-N6 & 0.175 & -0.176 & 0.175 & -0.180 & 0.000 & 0.004 \\
\hline WUXPAG $^{m}$ & $\mathrm{~S} 1-\mathrm{N} 6$ & 0.192 & -0.176 & 0.152 & -0.180 & 0.040 & 0.004 \\
\hline \multicolumn{8}{|l|}{$\mathrm{S} \cdots \mathrm{O}$} \\
\hline PAFVEY $^{s}$ & $\mathrm{~S} 1-\mathrm{O} 4$ & 0.151 & -0.232 & 0.176 & -0.250 & -0.025 & 0.018 \\
\hline WOCQEK $^{s}$ & $\mathrm{~S} 1-\mathrm{O} 4$ & 0.049 & -0.220 & 0.056 & -0.242 & -0.007 & 0.022 \\
\hline $\mathrm{ADOFEF}^{m *}$ & $\mathrm{~S} 4-\mathrm{O} 1$ & 0.004 & -0.291 & -0.006 & -0.305 & 0.011 & 0.014 \\
\hline $\mathrm{ADOFEF}^{m *}$ & S3-O1 & 0.419 & -0.291 & 0.417 & -0.305 & 0.001 & 0.014 \\
\hline MAVRAD $^{m}$ & $\mathrm{~S} 1-\mathrm{O} 2$ & 0.088 & -0.245 & 0.089 & -0.253 & -0.001 & 0.008 \\
\hline MEHNIY $^{m}$ & $\mathrm{~S} 4-\mathrm{O} 2$ & 0.071 & -0.282 & 0.047 & -0.209 & 0.024 & -0.073 \\
\hline NAHMUE $^{m}$ & $\mathrm{~S} 2-\mathrm{O} 4$ & 0.037 & -0.249 & 0.049 & -0.267 & -0.013 & 0.018 \\
\hline NAHMUE $^{m}$ & $\mathrm{~S} 1-\mathrm{O} 4$ & 0.038 & -0.249 & 0.049 & -0.267 & -0.011 & 0.018 \\
\hline PUDMUW $^{m}$ & $\mathrm{~S} 5-\mathrm{O} 4$ & 0.026 & -0.225 & 0.033 & -0.235 & -0.007 & 0.011 \\
\hline PUDMUW $^{m}$ & $\mathrm{~S} 1-\mathrm{O} 4$ & 0.085 & -0.225 & 0.078 & -0.235 & 0.007 & 0.011 \\
\hline QELQEE $^{m}$ & $\mathrm{~S} 1-\mathrm{O} 2$ & 0.062 & -0.237 & 0.072 & -0.249 & -0.010 & 0.012 \\
\hline QELQEE $^{m}$ & S2-O2 & 0.073 & -0.237 & 0.074 & -0.249 & -0.001 & 0.012 \\
\hline ZAVHEJ $^{m *}$ & S1-O3 & 0.070 & -0.235 & 0.060 & -0.241 & 0.010 & 0.006 \\
\hline \multicolumn{8}{|l|}{$\mathrm{Se} \cdots \mathrm{N}$} \\
\hline BESEAZ01 $^{s}$ & Se1-N4 & 0.250 & -0.172 & 0.258 & -0.182 & -0.008 & 0.010 \\
\hline FENFIO $^{s}$ & Se1-N4 & 0.109 & -0.051 & 0.112 & -0.080 & -0.003 & 0.030 \\
\hline WERYAT $^{s}$ & Se1-N3 & 0.131 & -0.104 & 0.153 & -0.136 & -0.022 & 0.032 \\
\hline $\mathrm{NECZUQ}^{m *}$ & Se3-N1 & 0.148 & -0.142 & 0.158 & -0.159 & -0.010 & 0.017 \\
\hline $\mathrm{SECNBZ}^{m}$ & Se1-N4 & 0.153 & -0.174 & 0.158 & -0.198 & -0.006 & 0.024 \\
\hline \multicolumn{8}{|l|}{$\mathrm{Se} \cdots \mathrm{O}$} \\
\hline BOJCOS $^{m *}$ & Se1-O3 & 0.086 & -0.266 & 0.080 & -0.277 & 0.006 & 0.012 \\
\hline LEDGAD $^{m}$ & Se1-O5 & 0.199 & -0.219 & 0.192 & -0.213 & 0.007 & -0.006 \\
\hline LEDGAD $^{m}$ & Se3-O1 & 0.199 & -0.214 & 0.192 & -0.213 & 0.007 & -0.002 \\
\hline LEVJOM $^{m}$ & $\mathrm{Se} 1-\mathrm{O} 4$ & 0.032 & -0.278 & 0.050 & -0.305 & -0.019 & 0.027 \\
\hline MUSCIM $^{m}$ & Se1-O2 & 0.071 & -0.278 & 0.072 & -0.310 & -0.002 & 0.032 \\
\hline
\end{tabular}




\section{S4 Interaction energies for hydrogen bonds, halogen bonds and chalcogen bonds}

In this section we present the values of interaction energies in kcal/mol for the hydrogen bonds (Table S6), halogen bonds (Table S7), and chalcogen bonds (Table S8). Interaction energies were calculated at M062x/Def2TZVP level. The atomic and molecular Roby-Gould bond indices are also given. 
Table S6: Interaction energy (kcal/mol) for hydrogen bonds $(\mathrm{D}-\mathrm{H} \cdots \mathrm{A}, \mathrm{D}=\mathrm{C}, \mathrm{N}, \mathrm{O}, \mathrm{A}=\mathrm{N}, \mathrm{O})$. Interaction energies were calculated at M062x/Def2TZVP level. The single and multipliable contact interactions in dimers are marked with superscript $s$ and $m$ respectively (and $m *$ for those that have two identical interactions within one dimer).

\begin{tabular}{|c|c|}
\hline $\begin{array}{l}\mathrm{D}-\mathrm{H} \cdots \mathrm{A} \\
\mathrm{CSD} \text { code }\end{array}$ & $\begin{array}{l}\text { Interaction energy } \\
(\mathrm{kcal} / \mathrm{mol})\end{array}$ \\
\hline \multicolumn{2}{|l|}{$\mathrm{C}-\mathrm{H} \cdots \mathrm{N}$} \\
\hline CYACHZ01 $^{s}$ & 3.15 \\
\hline DMSDIM01 $^{s}$ & 2.03 \\
\hline MEADEN02 $^{s}$ & 1.96 \\
\hline TRAZOL02 $^{s}$ & 2.76 \\
\hline BUGKIX01N ${ }^{m}$ & 2.00 \\
\hline BUGKIX01 ${ }^{m}$ & 5.13 \\
\hline CYACHZ01 ${ }^{m}$ & 1.64 \\
\hline NURWOM02 $^{m *}$ & 3.67 \\
\hline NURWOM02 ${ }^{m}$ & 3.04 \\
\hline TEPNIT0 $^{m *}$ & 5.94 \\
\hline${\mathrm{XEHMOM} 01^{m}}$ & 2.06 \\
\hline${\mathrm{XEHMOM} 01^{m}}^{m}$ & 2.26 \\
\hline \multicolumn{2}{|l|}{$\mathrm{N}-\mathrm{H} \cdots \mathrm{N}$} \\
\hline BAZGOY0 $5^{s}$ & 2.03 \\
\hline DMSDIM01 $^{s}$ & 4.91 \\
\hline FORAMO01 $^{s}$ & 4.15 \\
\hline IMAZOL06 $^{s}$ & 9.19 \\
\hline TRAZOL02 $^{s}$ & 8.41 \\
\hline \multicolumn{2}{|l|}{$\mathrm{O}-\mathrm{H} \cdots \mathrm{N}$} \\
\hline FORAMO01s & 7.35 \\
\hline GLOXIM11 $^{s}$ & 7.18 \\
\hline${\text { MAMPOL } 02^{s}}^{s}$ & 7.53 \\
\hline LOLSUA $^{m}$ & 18.50 \\
\hline ULAWAF $05^{m}$ & 27.45 \\
\hline \multicolumn{2}{|l|}{$\mathrm{C}-\mathrm{H} \cdots \mathrm{O}$} \\
\hline ABINOR04 ${ }^{s}$ & 2.12 \\
\hline NBONAN01 ${ }^{s}$ & 1.12 \\
\hline POKKAD01 ${ }^{s}$ & 1.83 \\
\hline $\mathrm{CYACHZ01}^{m *}$ & 13.37 \\
\hline FACETA01 ${ }^{m *}$ & 5.27 \\
\hline GLYGLY0 $^{m}$ & 16.86 \\
\hline METURA01 ${ }^{m}$ & 4.96 \\
\hline \multicolumn{2}{|l|}{$\mathrm{N}-\mathrm{H} \cdots \mathrm{O}$} \\
\hline BZAMID02 $2^{m *}$ & 15.86 \\
\hline CYURAC $12^{m *}$ & 14.02 \\
\hline FACETA01 $1^{m *}$ & 16.38 \\
\hline ULAWAF $05^{m *}$ & 16.28 \\
\hline UROXAL01 ${ }^{m *}$ & 18.79 \\
\hline $\mathrm{ZECWUB} 1^{m *}$ & 27.38 \\
\hline \multicolumn{2}{|l|}{$\mathrm{O}-\mathrm{H} \cdots \mathrm{O}$} \\
\hline BESKAL10 ${ }^{m *}$ & 18.65 \\
\hline CUKCAM18 $8^{m *}$ & 22.07 \\
\hline SALIAC $12^{m *}$ & 19.02 \\
\hline $\mathrm{SUCACB} 3^{m *}$ & 20.28 \\
\hline ZZZEEU05 $5^{m *}$ & 20.48 \\
\hline
\end{tabular}


Table S7: Interaction energy (kcal/mol) for halogen bonds $(\mathrm{X} \cdots \mathrm{A}, \mathrm{X}=\mathrm{Cl}, \mathrm{Br}, \mathrm{A}=\mathrm{N}, \mathrm{O})$. Interaction energies were calculated at M062x/Def2TZVP level. The single and multipliable contact interactions in dimers are marked with superscript $s$ and $m$ respectively (and $m *$ for those that have two identical interactions within one dimer).

\begin{tabular}{|c|c|}
\hline $\begin{array}{l}\mathrm{X} \cdots \mathrm{A} \\
\mathrm{CSD} \text { code }\end{array}$ & $\begin{array}{c}\text { Interaction energy } \\
(\mathrm{kcal} / \mathrm{mol})\end{array}$ \\
\hline \multicolumn{2}{|l|}{$\mathrm{Cl} \cdots \mathrm{N}$} \\
\hline $\mathrm{CCACENN}^{s}$ & 2.99 \\
\hline${\text { DESKER } 01^{s}}^{s}$ & 0.91 \\
\hline NABZAS $^{s}$ & 2.44 \\
\hline PCLPYR $^{s}$ & 2.65 \\
\hline VUGSIZ $^{s}$ & 1.79 \\
\hline PALPAV $^{m}$ & 2.44 \\
\hline $\mathrm{XIZPON}^{m}$ & 1.35 \\
\hline \multicolumn{2}{|l|}{$\mathrm{Cl} \cdots \mathrm{O}$} \\
\hline $\mathrm{BEDMONN}^{s}$ & 1.46 \\
\hline BZQDCL11 $^{s}$ & 1.53 \\
\hline $\mathrm{CORDUI}^{s}$ & 0.64 \\
\hline $\mathrm{DCLBZQ}{ }^{s}$ & 1.48 \\
\hline IRUFEH01 $^{s}$ & 1.56 \\
\hline JOJTIL $^{s}$ & 2.30 \\
\hline RUBSUD $^{s}$ & 1.56 \\
\hline${\text { TCACAD } 01^{s}}^{s}$ & 1.28 \\
\hline GEXWUB $^{s}$ & 1.74 \\
\hline PEPFUL $^{s}$ & 1.39 \\
\hline \multicolumn{2}{|l|}{$\mathrm{Br} \cdots \mathrm{N}$} \\
\hline $\mathrm{BCACENN}^{s}$ & 3.88 \\
\hline BONFIT $^{s}$ & 3.12 \\
\hline $\mathrm{QONHUX}^{s}$ & 2.36 \\
\hline RIRFOON $^{s}$ & 1.71 \\
\hline $\mathrm{KUYCUD}^{s}$ & 2.83 \\
\hline \multicolumn{2}{|l|}{$\mathrm{Br} \cdots \mathrm{O}$} \\
\hline BMLTAAN $^{s}$ & 2.18 \\
\hline $\mathrm{CIRSONN}^{s}$ & 0.98 \\
\hline $\mathrm{JEVVOW}^{s}$ & 3.00 \\
\hline $\mathrm{VAQXUG}^{s}$ & 2.11 \\
\hline VEWTAU $^{s}$ & 2.49 \\
\hline VEWTEY $^{s}$ & 1.46 \\
\hline VITVEZ $^{s}$ & 2.50 \\
\hline WADFIR $^{s}$ & 1.96 \\
\hline ACETBR02 ${ }^{m}$ & 5.59 \\
\hline
\end{tabular}


Table S8: Interaction energy $(\mathrm{kcal} / \mathrm{mol})$ for chalcogen bonds $(\mathrm{Y} \cdots \mathrm{A}, \mathrm{Y}=\mathrm{S}, \mathrm{Se}, \mathrm{A}=\mathrm{N}, \mathrm{O})$. Interaction energies were calculated at M062x/Def2TZVP level. The single and multipliable contact interactions in dimers are marked with superscript $s$ and $m$ respectively (and $m *$ for those that have two identical interactions within one dimer).

\begin{tabular}{|c|c|}
\hline $\begin{array}{l}\text { Y } \cdots A \\
\text { CSD code }\end{array}$ & $\begin{array}{c}\text { Interaction energy } \\
(\mathrm{kcal} / \mathrm{mol})\end{array}$ \\
\hline \multicolumn{2}{|l|}{$\mathrm{S} \cdots \mathrm{N}$} \\
\hline CEBYUD $^{s}$ & 2.29 \\
\hline QOBFUI $^{s}$ & 2.65 \\
\hline SAZCEC $^{s}$ & 2.23 \\
\hline GEDHAY $^{m}$ & 6.18 \\
\hline IFULUQ04 ${ }^{m}$ & 23.94 \\
\hline WASHEE $^{m}$ & 23.73 \\
\hline WUXPAG $^{m}$ & 33.27 \\
\hline \multicolumn{2}{|l|}{$\mathrm{S} \cdots \mathrm{O}$} \\
\hline PAFVEY $^{s}$ & 2.93 \\
\hline WOCQEK $^{s}$ & 6.01 \\
\hline $\mathrm{ADOFEF}^{m *}$ & 5.05 \\
\hline MAVRAD $^{m}$ & 7.57 \\
\hline MEHNIY $^{m}$ & 35.52 \\
\hline NAHMUE $^{m}$ & 3.61 \\
\hline PUDMUW $^{m}$ & 2.97 \\
\hline QELQEE $^{m}$ & 4.79 \\
\hline ZAVHEJ $^{m *}$ & 4.30 \\
\hline \multicolumn{2}{|l|}{$\mathrm{Se} \cdots \mathrm{N}$} \\
\hline BESEAZ01 $^{s}$ & 3.06 \\
\hline FENFION $^{s}$ & 3.87 \\
\hline WERYAT $^{s}$ & 4.53 \\
\hline NECZUQ $^{m *}$ & 8.75 \\
\hline SECNBZ $^{m}$ & 6.61 \\
\hline \multicolumn{2}{|l|}{$\mathrm{Se} \cdots \mathrm{O}$} \\
\hline $\operatorname{BOJCOS}^{m *}$ & 4.88 \\
\hline LEDGAD $^{m}$ & 6.11 \\
\hline LEVJOM $^{m}$ & 4.57 \\
\hline MUSCIM $^{m}$ & 5.67 \\
\hline
\end{tabular}




\section{S5 Testing the conservation of Roby-Gould bond bond order}

Table S9: Total $(\mathrm{D}-\mathrm{X})$ and $(\mathrm{A} \cdots \mathrm{X})$ atom $\cdots$ atom Roby-Gould bond indices $(\tau)$ for 15 selected dimers and corresponding monomers $(\tau(\mathrm{D}-\mathrm{X})$ and $\tau(\mathrm{A} \cdots \mathrm{X})$ respectively). The sum of the $\mathrm{D}-\mathrm{X}$ and $\mathrm{A} \cdots \mathrm{X}$ bond indices in dimers $\left(\tau(\mathrm{D}-\mathrm{A}+\mathrm{A} \cdots \mathrm{X})_{\text {Dimer }}\right)$ and the differences in $\mathrm{D}-\mathrm{X}$ bond index between dimers and monomers $\left(\tau\left(\Delta_{\mathrm{D}-\mathrm{X}}\right)\right)$ are also given. Wavefunctions optimized at the M062x/Def2SVP level.

\begin{tabular}{|c|c|c|c|c|c|c|}
\hline CSD code & Interaction & $\tau(\mathrm{D}-\mathrm{X})_{\text {Monomer }}$ & $\tau(\mathrm{D}-\mathrm{X})_{\text {Dimer }}$ & $\tau(\mathrm{A} \cdots \mathrm{X})_{\text {Dimer }}$ & $\tau(\mathrm{D}-\mathrm{A}+\mathrm{A} \cdots \mathrm{X})_{\text {Dimer }}$ & $\tau\left(\Delta_{\mathrm{D}-\mathrm{X}}\right)$ \\
\hline IMAZOL06 & $\mathrm{N}-\mathrm{H} \cdots \mathrm{N}$ & 0.942 & 0.814 & 0.272 & 1.086 & 0.128 \\
\hline FORAMO01 & $\mathrm{O}-\mathrm{H} \cdots \mathrm{N}$ & 0.945 & 0.784 & 0.338 & 1.121 & 0.162 \\
\hline ULAWAF05 & $\mathrm{O}-\mathrm{H} \cdots \mathrm{N}$ & 0.930 & 0.694 & 0.385 & 1.079 & 0.236 \\
\hline METURA01 & $\mathrm{C}-\mathrm{H} \cdots \mathrm{O}$ & 0.934 & 0.914 & 0.167 & 1.081 & 0.020 \\
\hline METURA01 & $\mathrm{C}-\mathrm{H} \cdots \mathrm{O}$ & 0.922 & 0.891 & 0.174 & 1.065 & 0.031 \\
\hline BZAMID02 & $\mathrm{N}-\mathrm{H} \cdots \mathrm{O}$ & 0.946 & 0.831 & 0.283 & 1.114 & 0.114 \\
\hline SUCACB03 & $\mathrm{O}-\mathrm{H} \cdots \mathrm{O}$ & 0.927 & 0.738 & 0.379 & 1.117 & 0.190 \\
\hline DESKER01 & $\mathrm{Cl} \cdots \mathrm{N}$ & 1.210 & 1.200 & 0.060 & 1.260 & 0.010 \\
\hline BCACEN & $\mathrm{Br} \cdots \mathrm{N}$ & 1.466 & 1.428 & 0.139 & 1.568 & 0.037 \\
\hline RIRFOO & $\mathrm{Br} \cdots \mathrm{N}$ & 1.150 & 1.130 & 0.140 & 1.270 & 0.020 \\
\hline ACETBR02 & $\mathrm{Br} \cdots \mathrm{O}$ & 0.739 & 0.695 & 0.170 & 0.865 & 0.044 \\
\hline CIRSON & $\mathrm{Br} \cdots \mathrm{O}$ & 1.140 & 1.130 & 0.120 & 1.250 & 0.010 \\
\hline JEVVOW & $\mathrm{Br} \cdots \mathrm{O}$ & 1.120 & 1.100 & 0.150 & 1.250 & 0.020 \\
\hline ZAVHEJ & $\mathrm{S} \cdots \mathrm{O}$ & 0.922 & 0.912 & 0.186 & 1.098 & 0.010 \\
\hline BOJCOS & $\mathrm{Se} \cdots \mathrm{O}$ & 0.946 & 0.926 & 0.221 & 1.146 & 0.020 \\
\hline
\end{tabular}




\section{S6 The angle dependence of RGBI values}

Two angles are most important in the discussion of the angle dependence of bond orders of a D-X . A intermolecular interaction, (i) $\angle \mathrm{D}-\mathrm{X} \cdots \mathrm{A}$ angle (ii) $\angle \mathrm{X} \cdots \mathrm{A}-\mathrm{C}$ angle. Among these, $\angle \mathrm{D}-\mathrm{X} \cdots \mathrm{A}$ angle is more significant as a nearly $180 \angle \mathrm{D}-\mathrm{X} \cdots \mathrm{A}$ angle is directly linked to the effective $n \rightarrow \sigma^{*}$ interaction. To test these we calculated and plotted the bond indices, total, covalent and ionic bond indices along with the AIM topological parameters at the bond critical points for a selected subset of interactions with $\mathrm{N}$ atom as the lone pair donor or bond acceptor (Table S10 and Figure S1). We find that these results are insufficient to show the directional sensitivity of RGBIs as compared to AIM topology. We have plotted total, covalent and ionic RGBIs for a subset of halogen and chalcogen bonded complexes versus $\angle \mathrm{D}-\mathrm{X} \cdots \mathrm{A}$ angle (Figure S1). No clear correlation could be found between these descriptors and the $\angle D-X \cdots A$ angle. It may also be noted that more complicated interaction angles around atom A and its hybridization state (as it is related to lone pair directionality) can also affect bond orders. The wide range of bond order values for interaction types such as XBs and YBs may be due to a variety of reasons and the bond angle directionality is only one of them. Chemical environment around the $\mathrm{X}$ and $\mathrm{A}$ atoms involved in the interaction, the partial charges of atoms $\mathrm{A}$ and $\mathrm{X}$ (even in monomer state) etc can influence the bond order values. However, we performed similar analysis on the hypothetical geometries for molecular dimers varying the interaction angles for the linear structures $\mathrm{N} \equiv \mathrm{C}-\mathrm{C} \equiv \mathrm{C}-\mathrm{Br}$ and $\mathrm{N} \equiv \mathrm{C}-\mathrm{C} \equiv \mathrm{C}-\mathrm{Cl}$ (CSD codes: BCACEN, and CCACEN respectively) which exhibit $\mathrm{Br} \cdots \mathrm{N}$ and $\mathrm{Cl} \cdots \mathrm{N}$ interactions. This analysis clearly brings out the differences in angle dependent trends in RGBIs vs AIM topological parameters.

Table S10: The angle dependence of total $(\tau)$, covalent $(c)$, and ionic $(i)$ Roby-Gould bond indices, and AIM topological parameters of a D-X ‥ A intermolecular interaction with respect to D-X ‥ A angle for a selected set of halogen and chalcogen bonded dimers. The distance (in $\AA$ ) and EML interaction energy (in kJ/mol) are also given.

\begin{tabular}{|c|c|c|c|c|c|c|c|c|}
\hline $\begin{array}{c}\text { A } \cdots X \\
\text { CSD code }\end{array}$ & $\begin{array}{c}d_{\mathrm{A} \cdots \mathrm{X}} \\
/ \stackrel{A}{A}\end{array}$ & $\begin{array}{c}\text { Angle }\left(\theta^{\circ}\right) \\
\angle D-X \cdots A\end{array}$ & $\begin{array}{c}\rho \\
/ b c p\end{array}$ & $\nabla^{2} \rho$ & $\begin{array}{c}\text { EML }(\mathrm{kJ} / \mathrm{mol}) \\
\text { interaction energy }\end{array}$ & $c$ & $i$ & $\tau$ \\
\hline \multicolumn{9}{|l|}{$\mathrm{Cl} \cdots \mathrm{A}$} \\
\hline $\mathrm{CCACEN}^{s}$ & 2.984 & 178.250 & 0.074 & 1.148 & -9.243 & 0.060 & 0.440 & 0.450 \\
\hline DESKER01 $^{s}$ & 2.954 & 163.710 & 0.091 & 1.275 & -11.580 & 0.070 & 0.350 & 0.360 \\
\hline $\mathrm{NABZAS}^{s}$ & 3.092 & 170.100 & 0.066 & 0.931 & -7.627 & 0.040 & 0.090 & 0.090 \\
\hline $\mathrm{PCLPYR}^{s}$ & 3.014 & 180.000 & & & & & & \\
\hline VUGSIZ $^{s}$ & 3.100 & 172.910 & 0.065 & 0.928 & -7.455 & 0.040 & 0.100 & 0.110 \\
\hline PALPAV $^{m}$ & 3.097 & 149.620 & 0.063 & 0.936 & -7.395 & 0.040 & 0.000 & 0.040 \\
\hline \multicolumn{9}{|l|}{$\mathrm{Br} \cdots \mathrm{N}$} \\
\hline $\mathrm{BCACEN}^{s}$ & 2.978 & 177.230 & 0.090 & 1.301 & -11.591 & 0.090 & 0.400 & 0.410 \\
\hline BONFIT $^{s}$ & 2.863 & 171.250 & 0.119 & 1.593 & -16.264 & 0.130 & 0.250 & 0.280 \\
\hline QONHUX $^{s}$ & 3.093 & 153.630 & 0.080 & 1.026 & -9.348 & 0.070 & 0.240 & 0.250 \\
\hline RIRFOO $^{s}$ & 3.164 & 167.460 & 0.072 & 0.920 & -8.063 & 0.060 & 0.260 & 0.260 \\
\hline \multicolumn{9}{|l|}{$\mathrm{S} \cdots \mathrm{N}$} \\
\hline $\mathrm{CEBYUD}^{s}$ & 3.050 & 149.490 & 0.079 & 0.998 & -9.037 & 0.080 & 0.070 & 0.110 \\
\hline $\mathrm{QOBFUI}^{s}$ & 2.992 & 170.010 & 0.088 & 1.101 & -10.443 & 0.080 & 0.030 & 0.090 \\
\hline $\mathrm{SAZCEC}^{s}$ & 3.096 & 171.920 & 0.073 & 0.917 & -8.126 & 0.080 & 0.050 & 0.090 \\
\hline GEDHAY $^{m}$ & 2.910 & 169.730 & 0.095 & 1.270 & -11.998 & 0.110 & 0.020 & 0.110 \\
\hline GEDHAY $^{m}$ & 3.086 & 153.830 & 0.075 & 1.011 & -8.762 & 0.080 & 0.060 & 0.100 \\
\hline IFULUQ04 $^{m}$ & 3.006 & 159.870 & 0.091 & 1.230 & -11.383 & 0.060 & 0.110 & 0.130 \\
\hline WASHEE $^{m}$ & 3.003 & 161.620 & 0.089 & 1.205 & -11.022 & 0.270 & 0.130 & 0.300 \\
\hline WASHEE $^{m}$ & 2.992 & 161.140 & 0.093 & 1.184 & -11.296 & 0.060 & 0.110 & 0.120 \\
\hline $\mathrm{WUXPAG}^{m}$ & 3.008 & 161.480 & 0.082 & 1.141 & -10.003 & 0.100 & 0.000 & 0.100 \\
\hline \multicolumn{9}{|l|}{$\mathrm{Se} \cdots \mathrm{N}$} \\
\hline BESEAZ01 $^{s}$ & 3.155 & 169.160 & 0.075 & 0.857 & -8.015 & 0.100 & 0.21 & 0.230 \\
\hline FENFIO $^{s}$ & 3.154 & 175.810 & 0.082 & 0.890 & -8.887 & 0.100 & 0.01 & 0.100 \\
\hline WERYAT $^{s}$ & 2.843 & 173.990 & 0.139 & 1.543 & -18.647 & 0.150 & 0.000 & 0.150 \\
\hline $\mathrm{NECZUQ}^{m *}$ & 2.877 & 168.200 & 0.124 & 1.379 & -15.953 & 0.180 & 0.150 & 0.230 \\
\hline $\mathrm{SECNBZ}^{m}$ & 3.058 & 174.740 & 0.083 & 1.050 & -9.718 & 0.100 & 0.140 & 0.170 \\
\hline
\end{tabular}



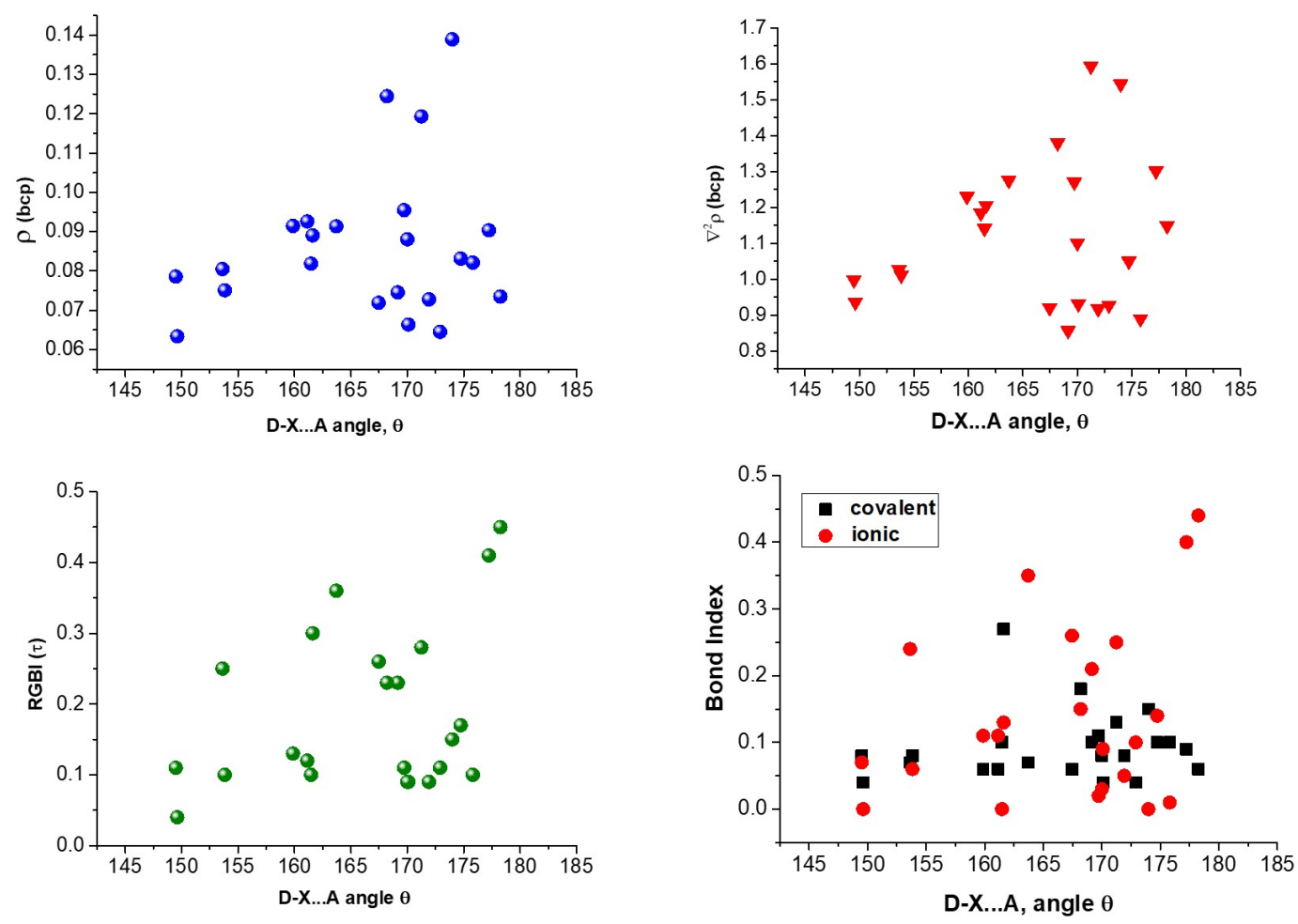

Figure S1: The angle dependence of total $(\tau)$, covalent $(c)$, and ionic $(i)$ Roby-Gould bond indices and AIM topological parameters of a $\mathrm{D}-\mathrm{X} \cdots \mathrm{A}$ intermolecular interaction with respect to $\mathrm{D}-\mathrm{X} \cdots \mathrm{A}$ angle for a selected set of halogen and chalcogen bonded dimers given in the Table S10. 


\section{S7 Percentage of covalency}

Figure S2 shows bond order-weighted average covalency percentages $\left(\% c_{W}\right)$ for atom $\cdots$ atom (light blue) and molecul...molecule bond indices (dark blue) for hydrogen, halogen and chalcogen bonds. For each interaction type, the average weighted covalency percentages were calculate using the formula:

$$
{ }_{0} c_{W}=\frac{\sum \tau_{i} . \% c_{i}}{\sum \tau_{i}}
$$

where $\tau_{i}$ and $\% c_{i}$ are the bond orders and percentage covalency of each interaction under XB, YB and HB.

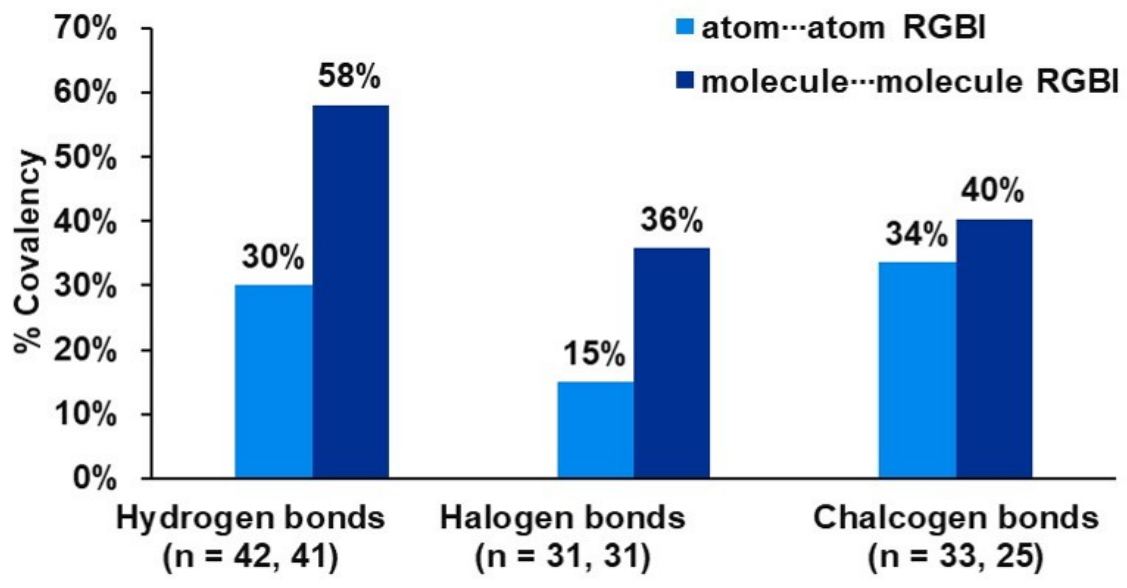

Figure S2: Bond order-weighted average covalency percentages $\left(\% c_{W}\right)$ for atom $\cdots$ atom (light blue) and molecul...molecule bond indices (dark blue) for hydrogen, halogen and chalcogen bonds. Number of interactions and molecular dimers studied for each type are given in parentheses. 


\section{S8 Molecular structures of selected dimers}

In this section we present the molecular structures of all dimers considered in this study with the hydrogen bonds, halogen bonds and chalcogen bonds. The distances in Angstrom are given for only those interactions concerned in this study.

These figures were generated from the crystallographic information files (CIF) in the Cambridge Crystallographic Structure Database (CSD) using CrystalExplorer program.

Please note that the molecular structures of all dimers are listed according to the same order where their CSD codes are listed in the Tables 2, 3 and S1.

The atoms of each dimer are labeled with different colored spheres. as follows:

- Hydrogen atom: yellow (small ball).

- Carbon atom: dark gray.

- Oxygen atom: red.

- Nitrogen atom: blue.

- chlorine atom: green.

- Bromine atom: gold.

- Sulfur atom: yellow (big ball).

- Selenium atom: light gray (big ball). 


\section{S8.1 Hydrogen bonds}

S8.1.1 $\quad \mathbf{C}-\mathbf{H} \cdots \mathrm{N}$ interaction

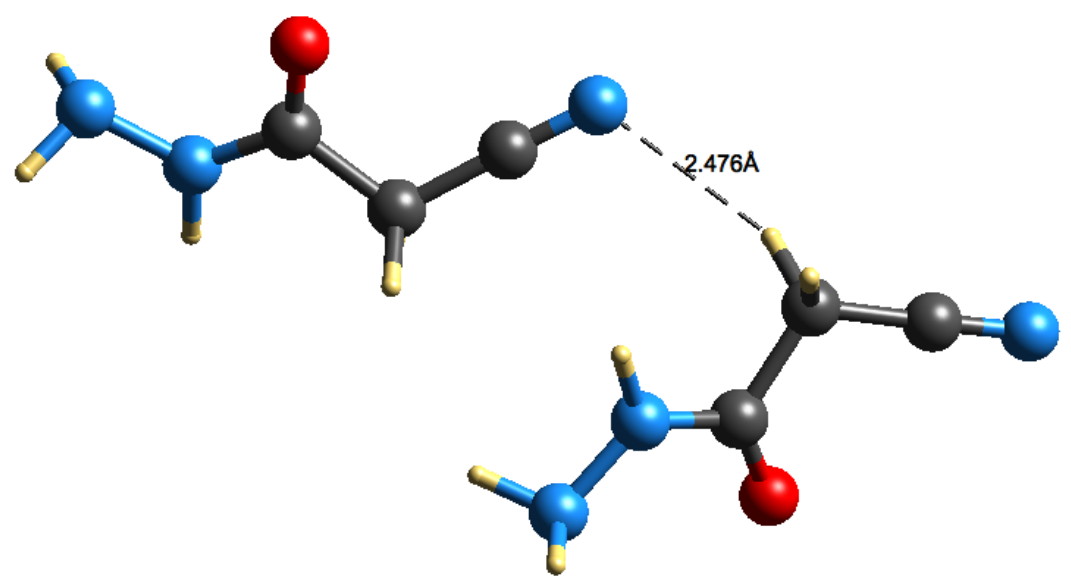

Figure S3: The molecular structure of CYACHZ01 ${ }^{s}$.

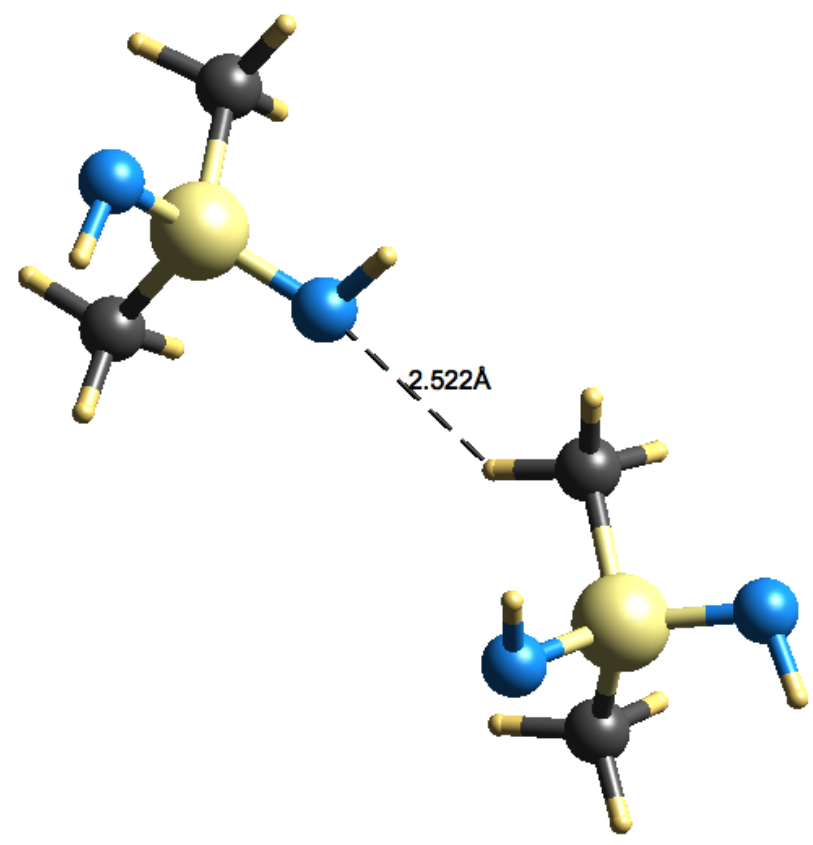

Figure S4: The molecular structure of DMSDIM01 ${ }^{s}$. 


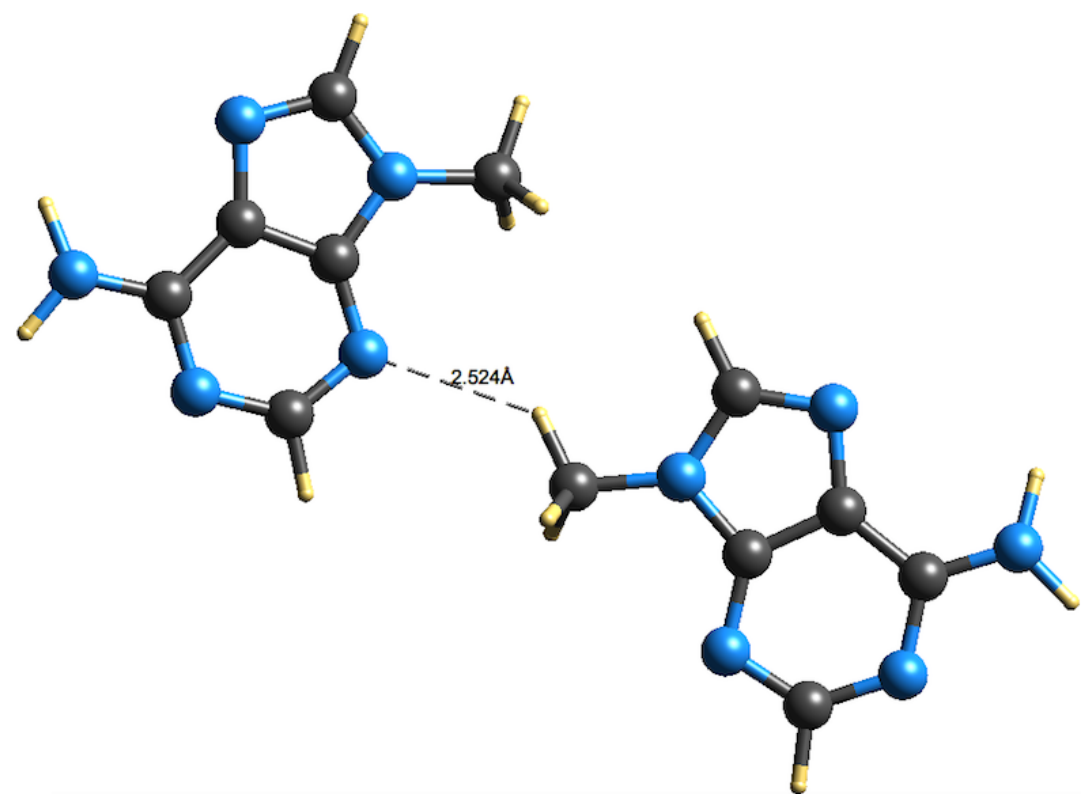

Figure S5: The molecular structure of MEADEN02 ${ }^{s}$.

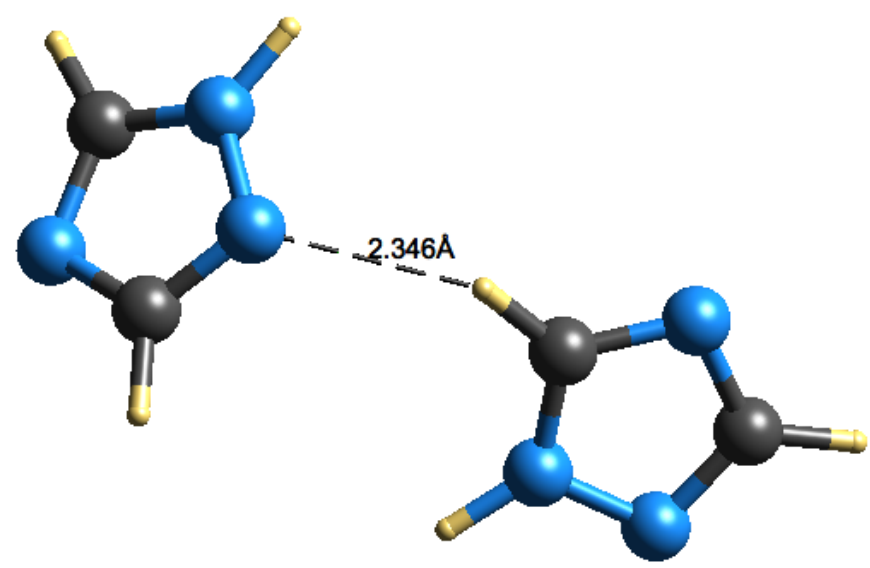

Figure S6: The molecular structure of TRAZOL02 ${ }^{s}$. 


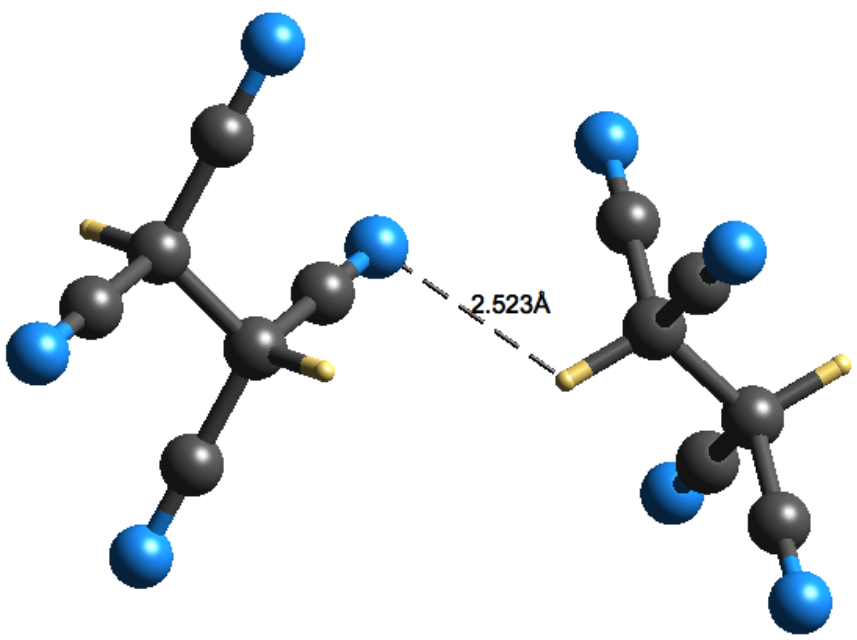

Figure S7: The molecular structure of BUGKIX01 ${ }^{s}$.

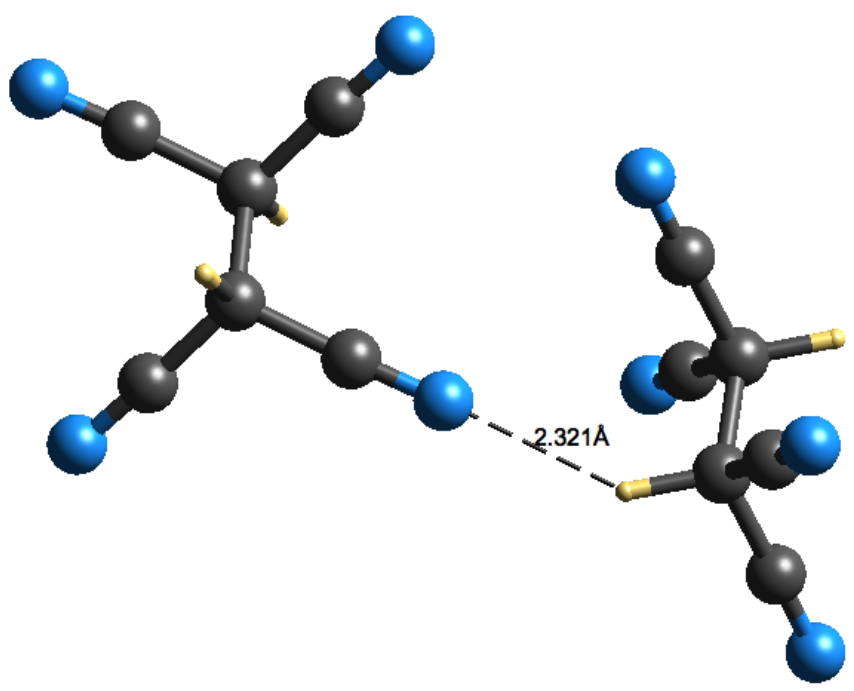

Figure S8: The molecular structure of BUGKIX01 ${ }^{s}$.

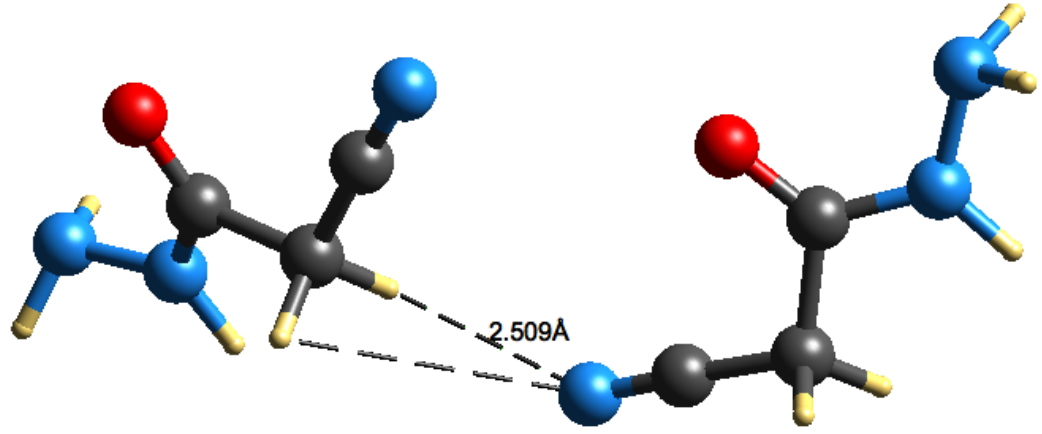

Figure S9: The molecular structure of CYACHZ01 ${ }^{m}$. 


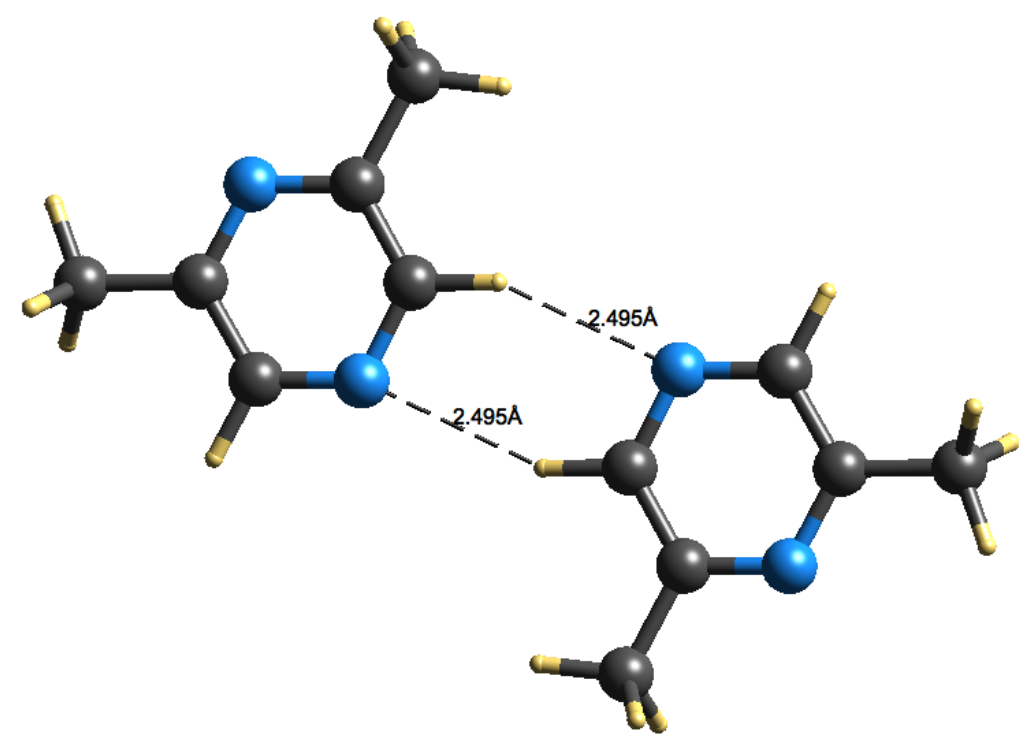

Figure S10: The molecular structure of NURWOM02 ${ }^{m *}$.

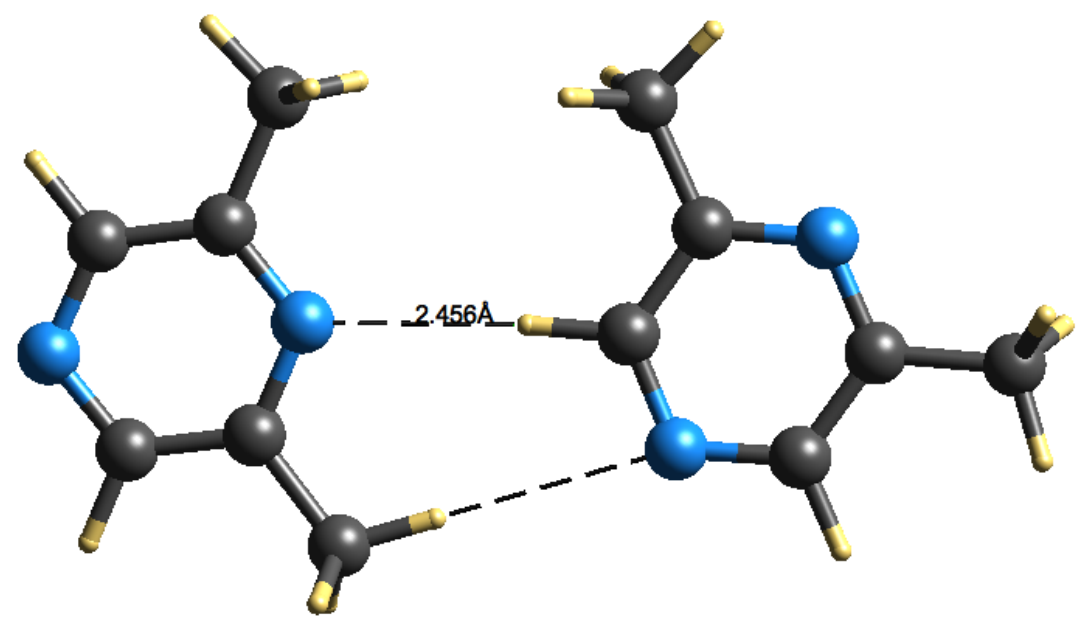

Figure S11: The molecular structure of NURWOM02 ${ }^{m}$.

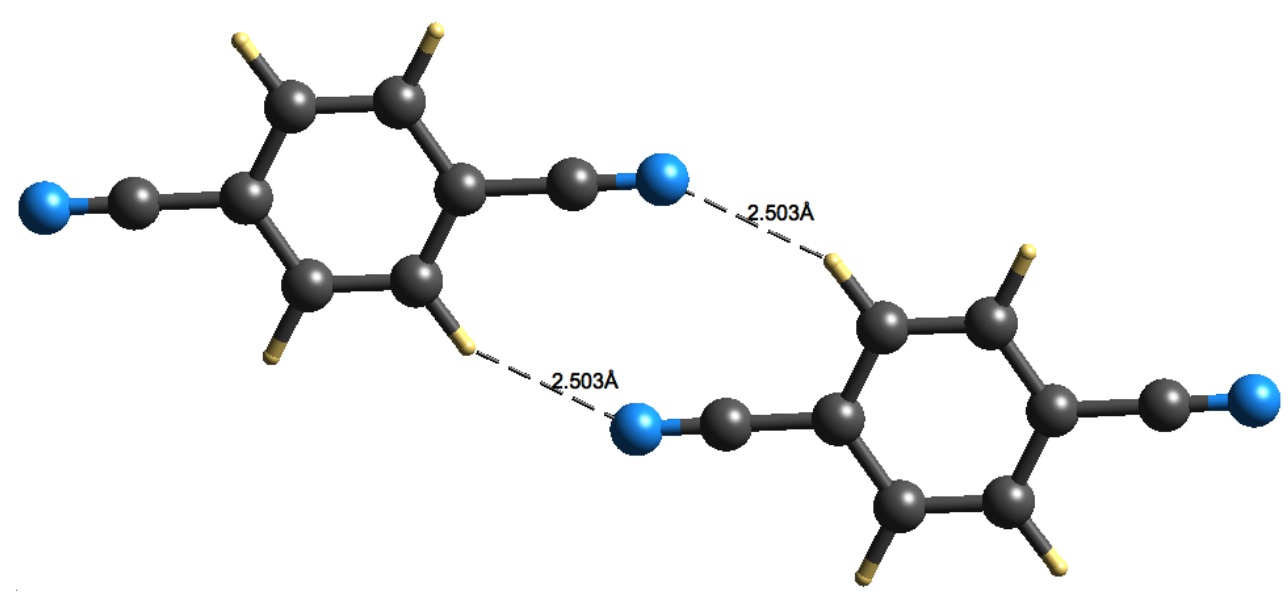

Figure S12: The molecular structure of TEPNIT04 ${ }^{m *}$. 


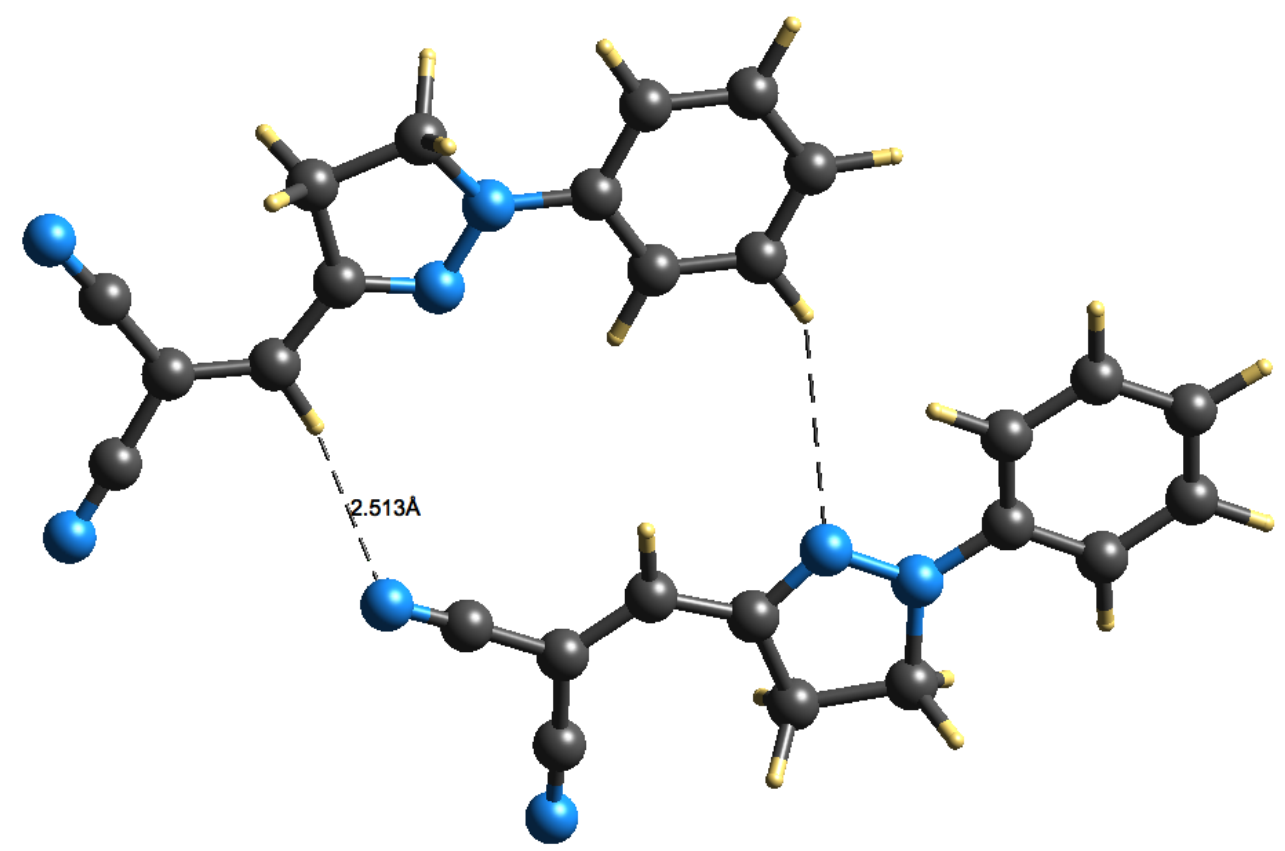

Figure S13: The molecular structure of XEHMOM01 ${ }^{m}$.

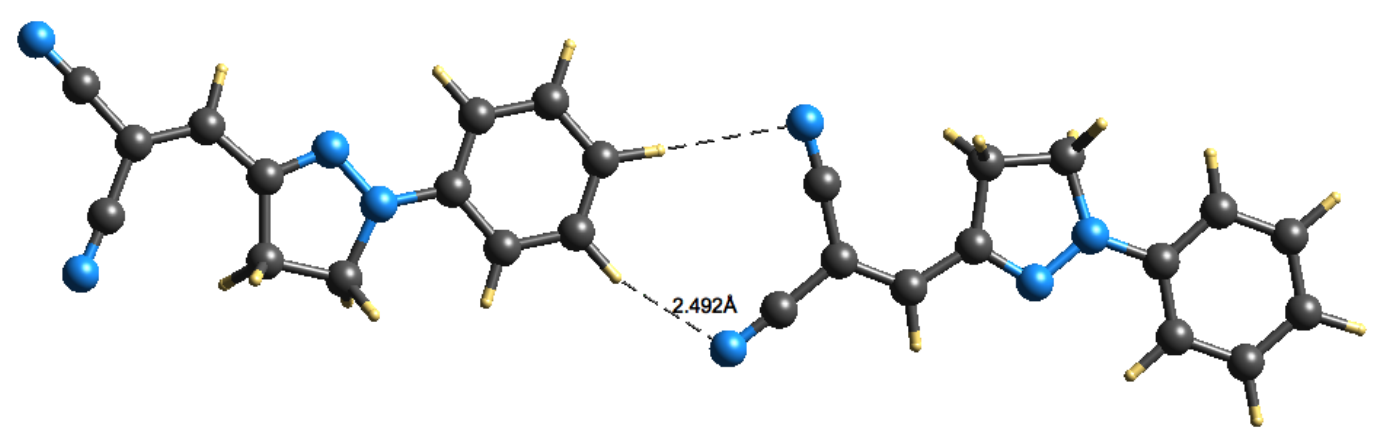

Figure S14: The molecular structure of XEHMOM01 ${ }^{m}$.

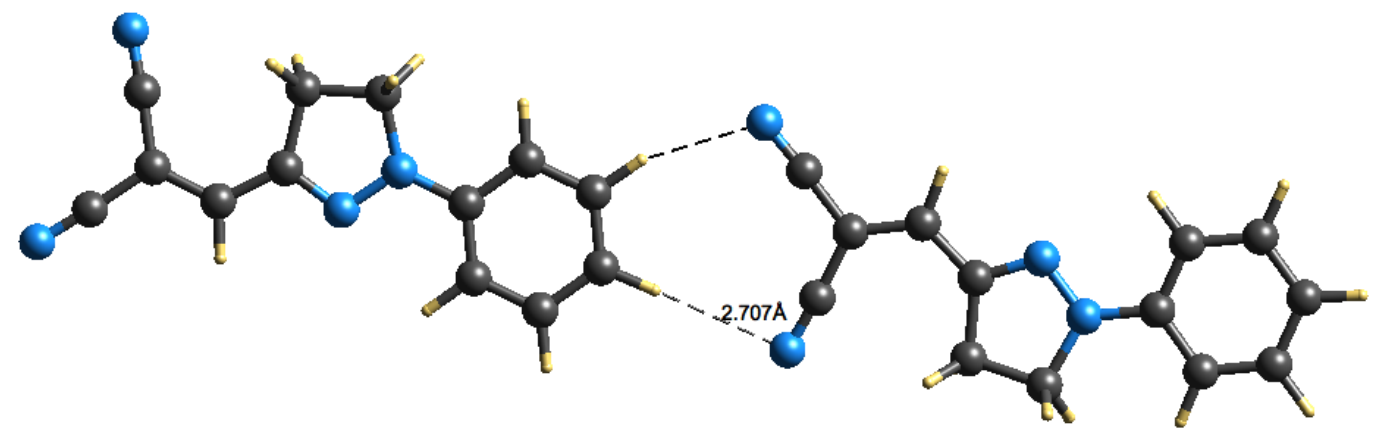

Figure S15: The molecular structure of XEHMOM01 ${ }^{m}$. 
S8.1.2 $\mathrm{N}-\mathrm{H} \cdots \mathrm{N}$ interactions

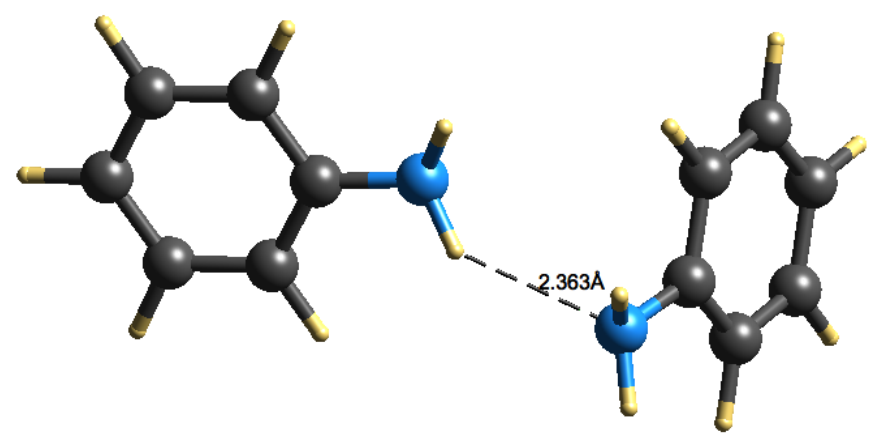

Figure S16: The molecular structure of BAZGOY05 ${ }^{s}$.

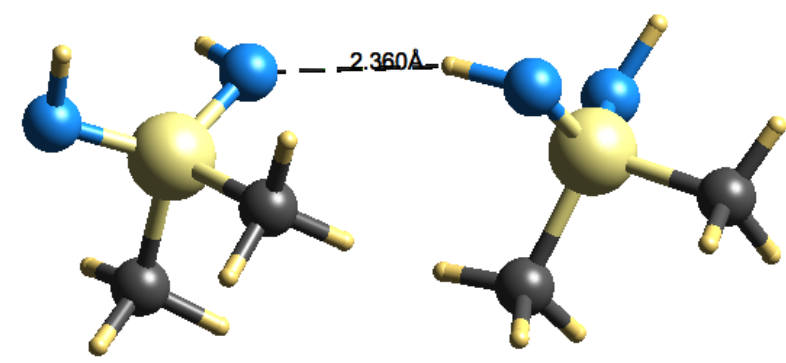

Figure S17: The molecular structure of DMSDIM01s ${ }^{\text {. }}$

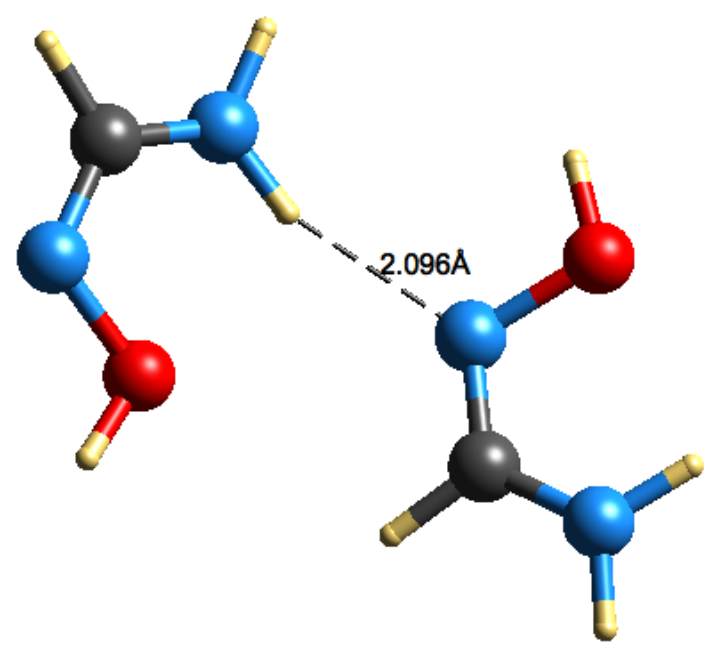

Figure S18: The molecular structure of FORAMO01 ${ }^{s}$. 


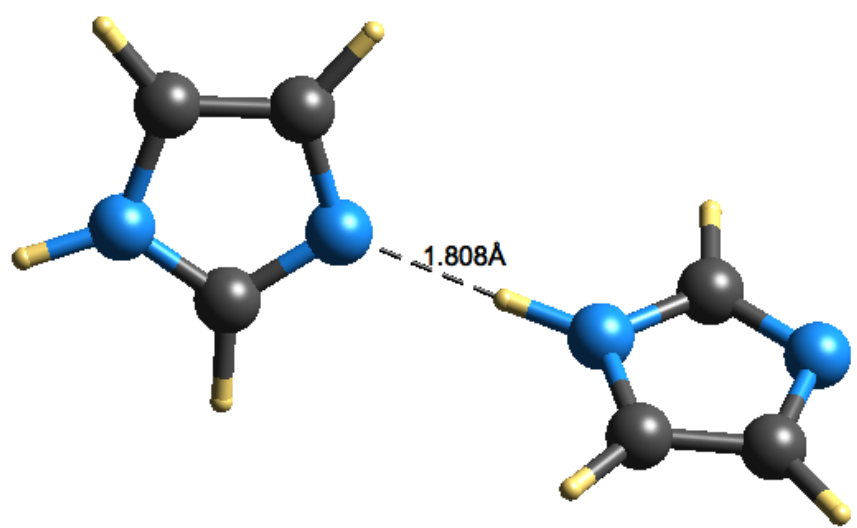

Figure S19: The molecular structure of IMAZOL06 ${ }^{s}$.

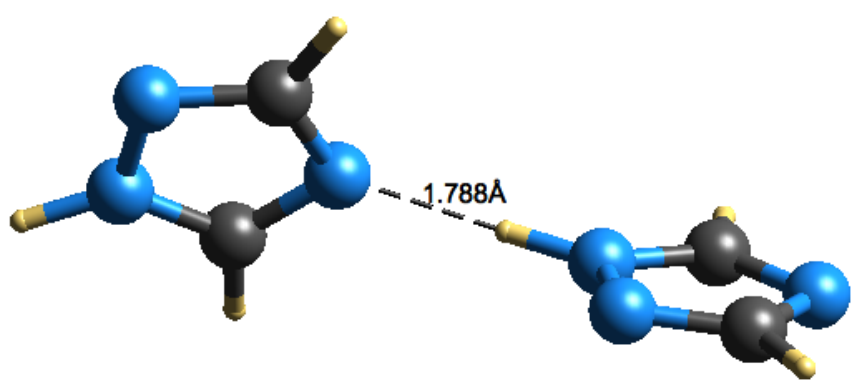

Figure S20: The molecular structure of TRAZOL02 ${ }^{s}$. 
S8.1.3 $\mathrm{O}-\mathrm{H} \cdots \mathrm{N}$ interactions

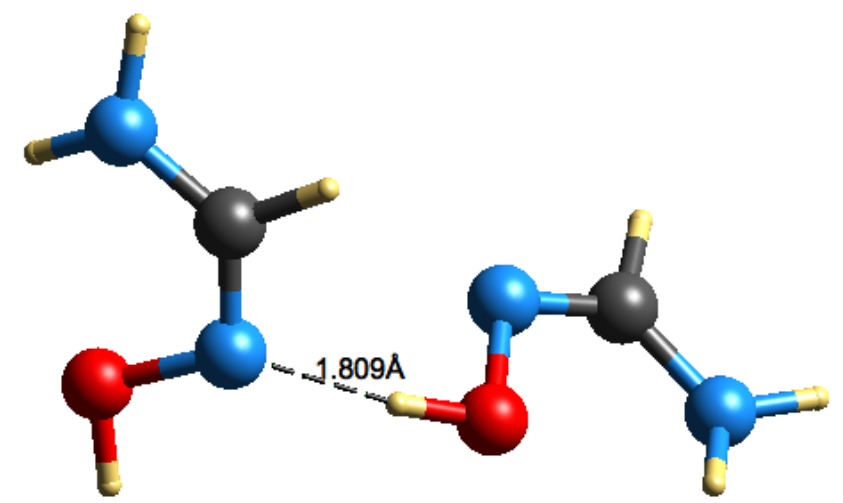

Figure S21: The molecular structure of FORAMO01 ${ }^{s}$.

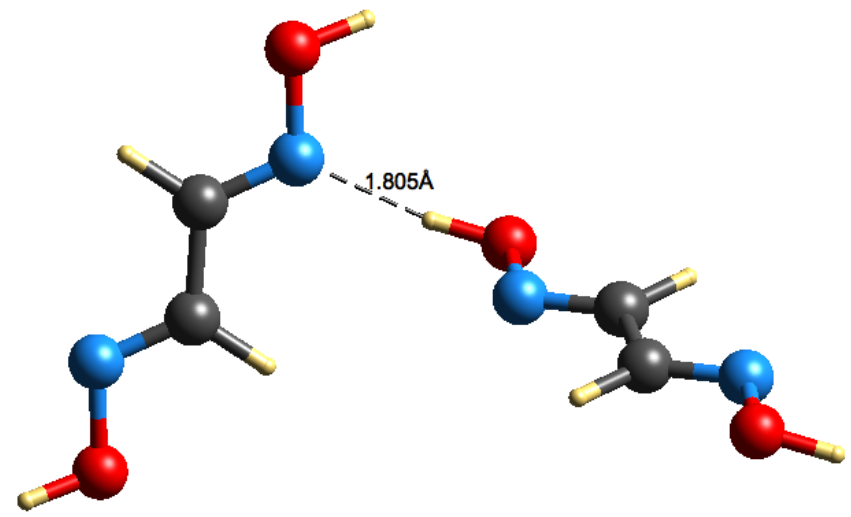

Figure S22: The molecular structure of GLOXIM11 ${ }^{s}$.

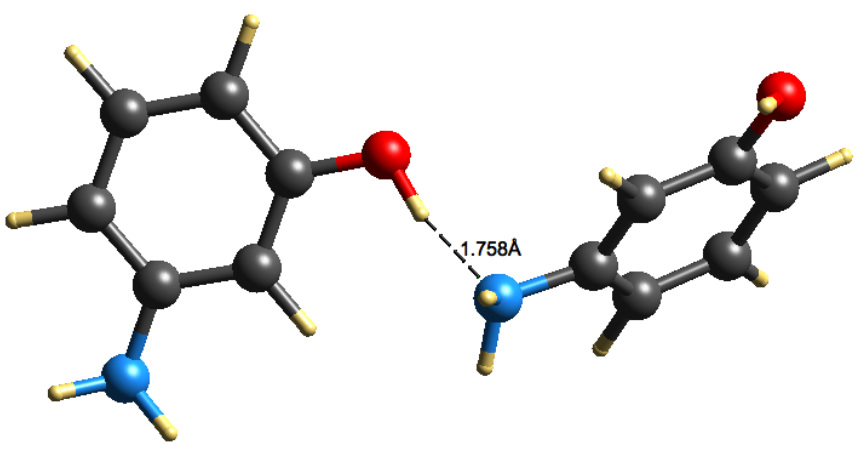

Figure S23: The molecular structure of MAMPOL02 ${ }^{s}$. 


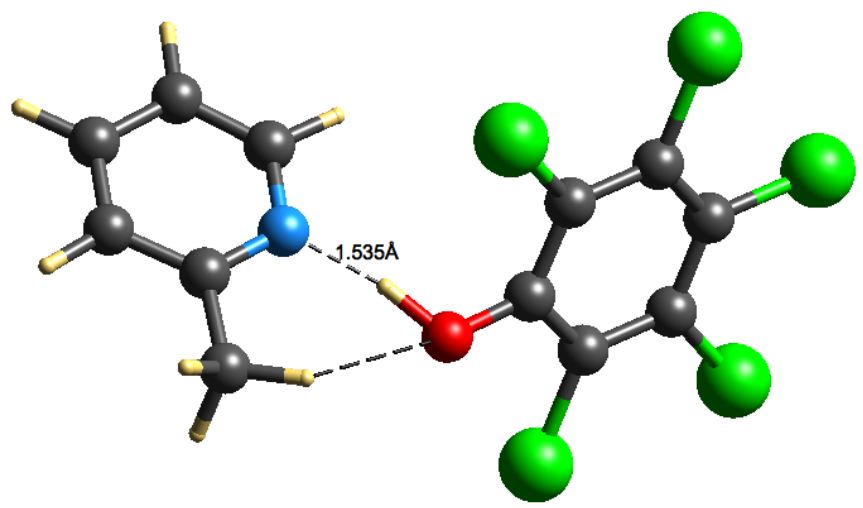

Figure S24: The molecular structure of LOLSUA ${ }^{m}$.

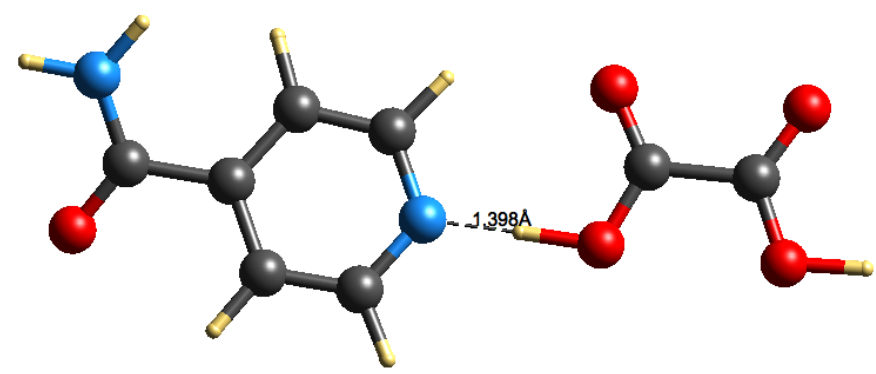

Figure S25: The molecular structure of ULAWAF0 $5^{m}$. 
S8.1.4 $\mathrm{C}-\mathrm{H} \cdots \mathrm{O}$ interactions

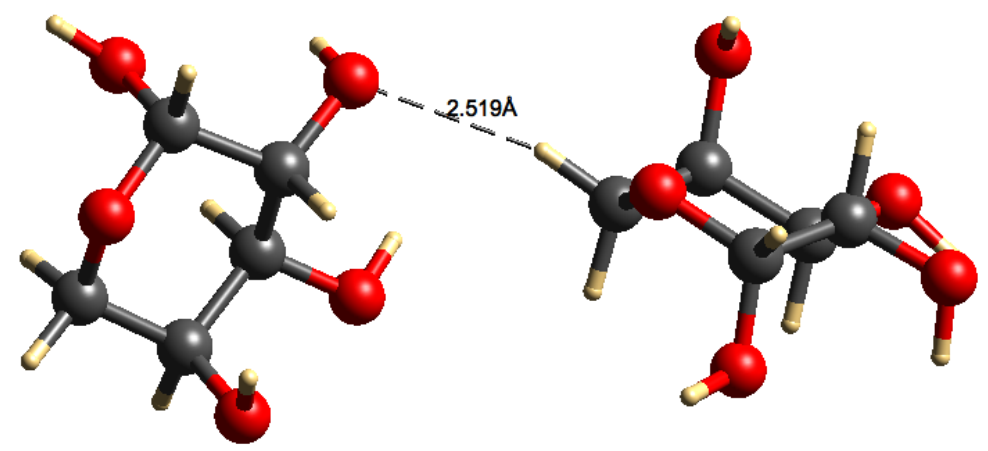

Figure S26: The molecular structure of ABINOR04 ${ }^{s}$.

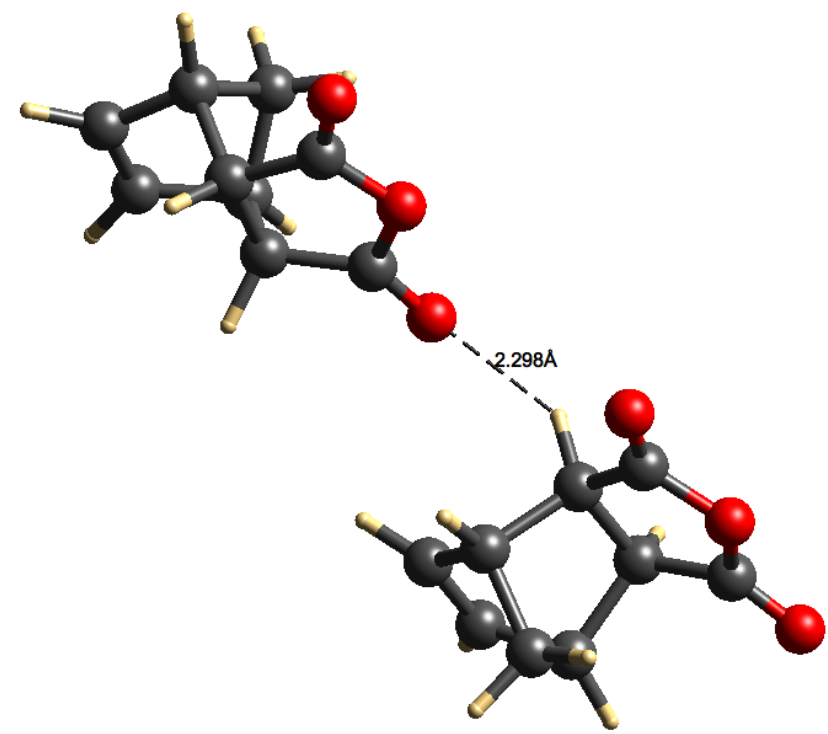

Figure S27: The molecular structure of NBONAN01 ${ }^{s}$.

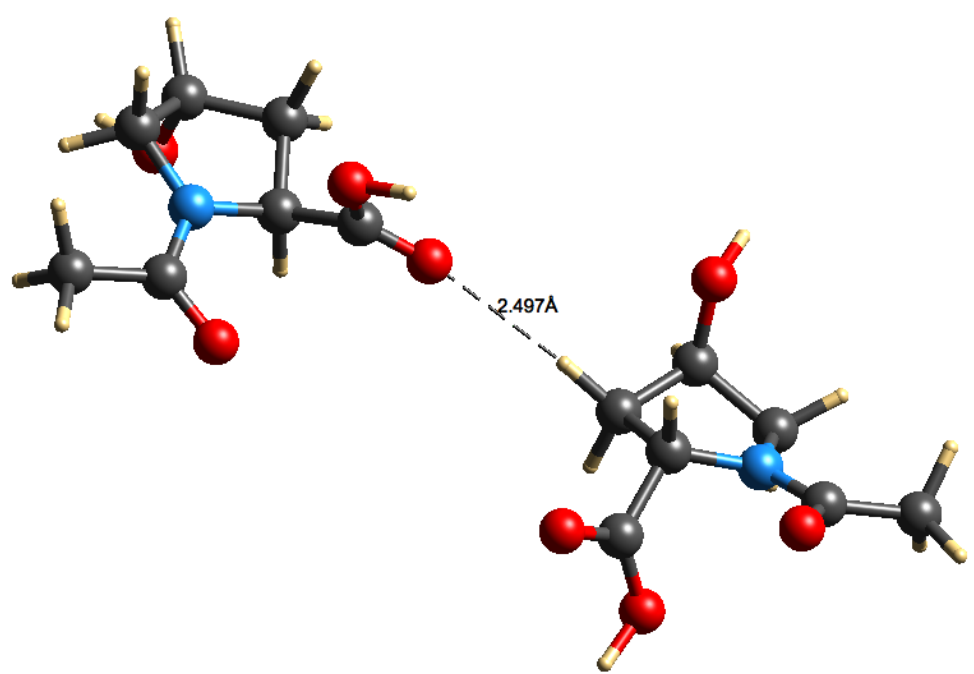

Figure S28: The molecular structure of POKKAD01 ${ }^{s}$. 


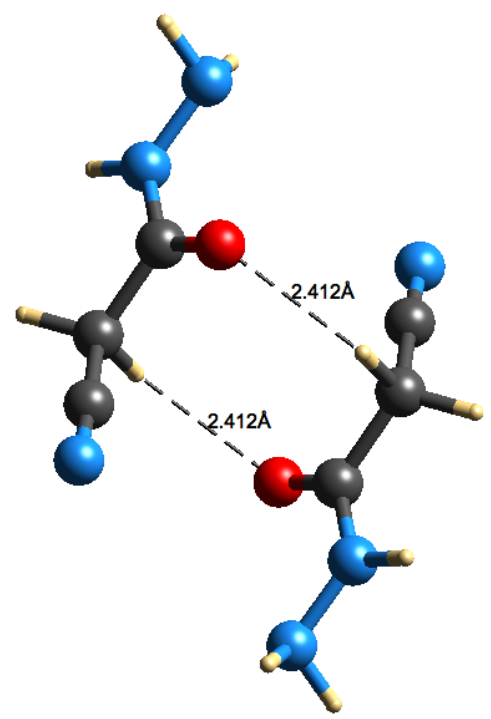

Figure S29: The molecular structure of CYACHZ01 ${ }^{m *}$

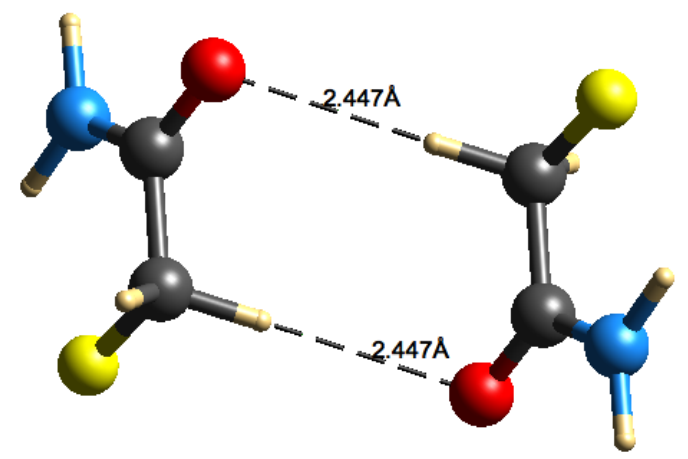

Figure S30: The molecular structure of FACETA01 ${ }^{m *}$.

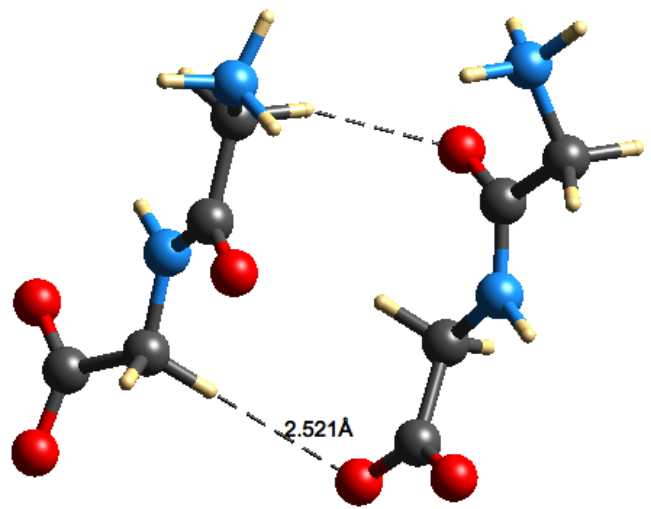

Figure S31: The molecular structure of GLYGLY0 ${ }^{m}$. 


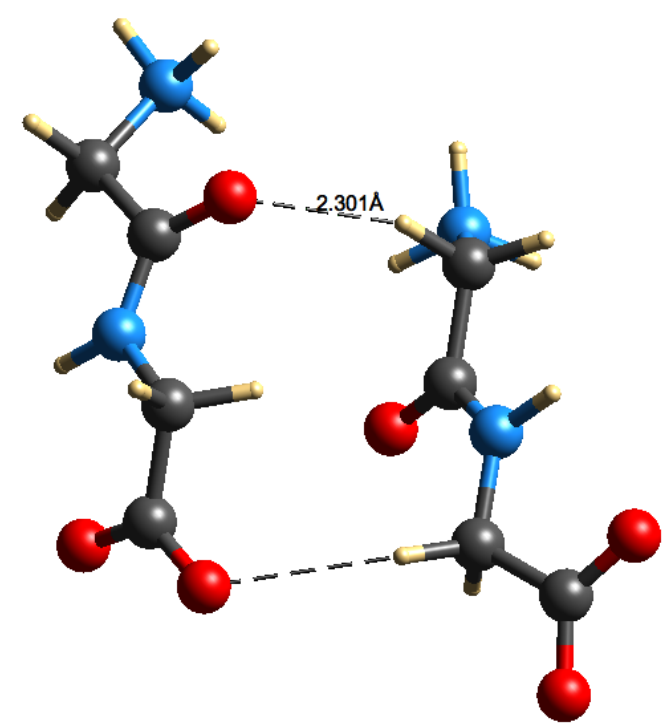

Figure S32: The molecular structure of GLYGLY04 ${ }^{m}$.

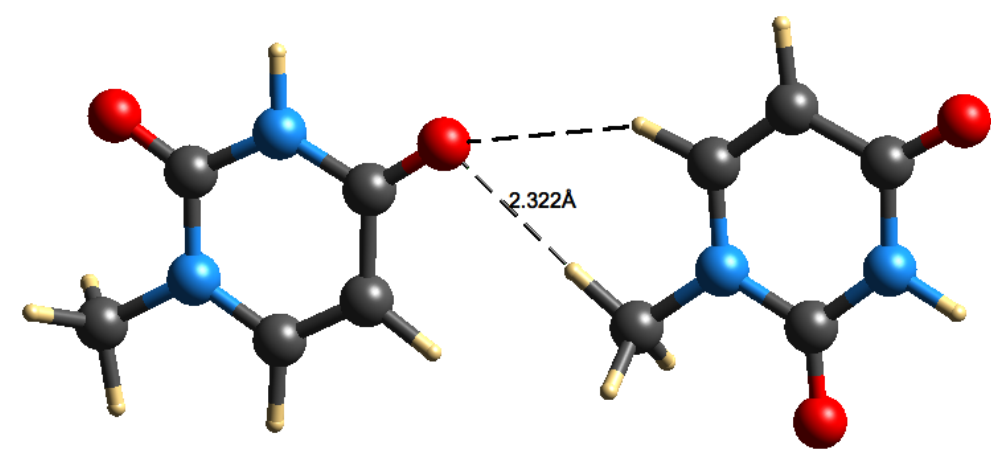

Figure S33: The molecular structure of METURA01 ${ }^{m}$. 
S8.1.5 $\mathrm{N}-\mathrm{H} \cdots \mathrm{O}$ interactions

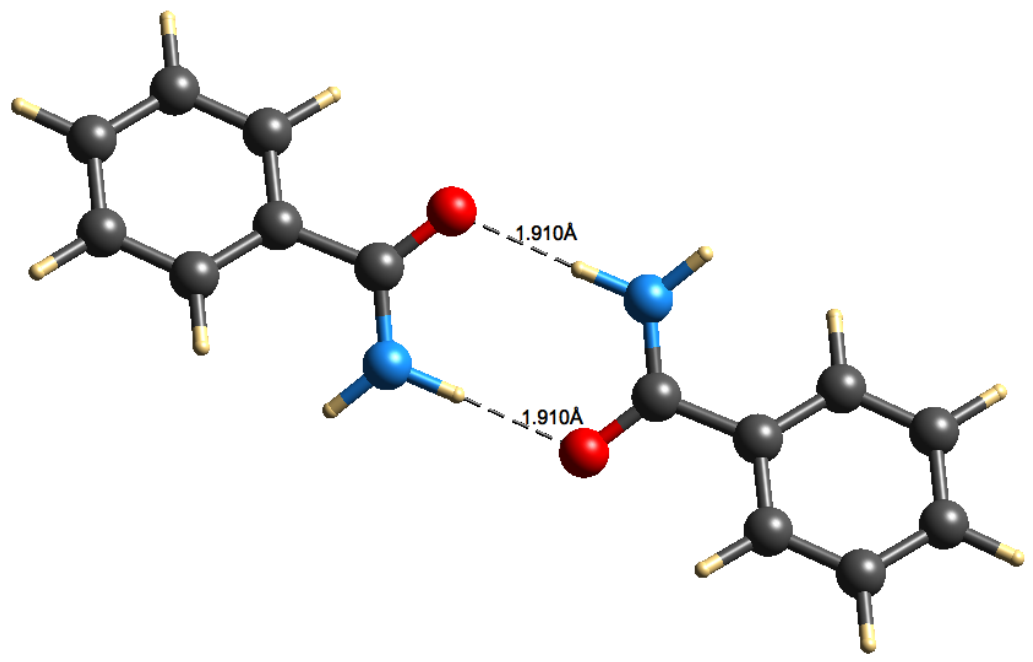

Figure S34: The molecular structure of BZAMID02 ${ }^{m *}$.

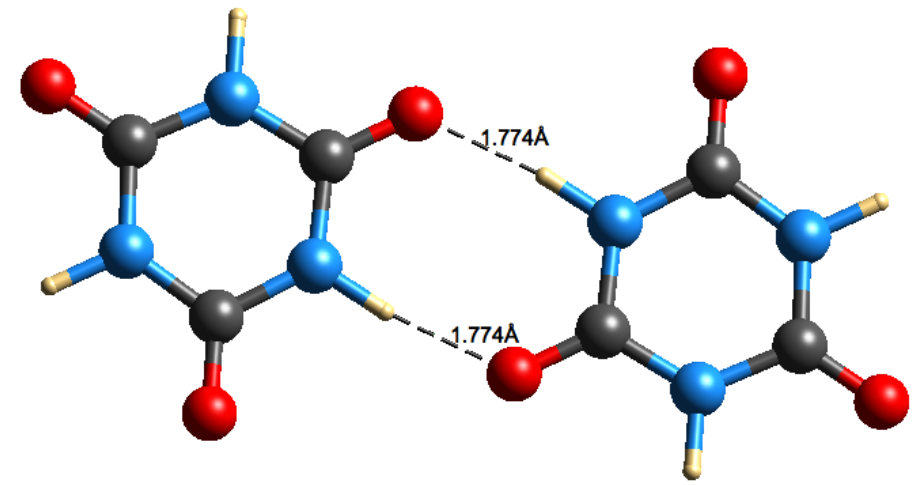

Figure S35: The molecular structure of CYURAC12 ${ }^{m *}$. 


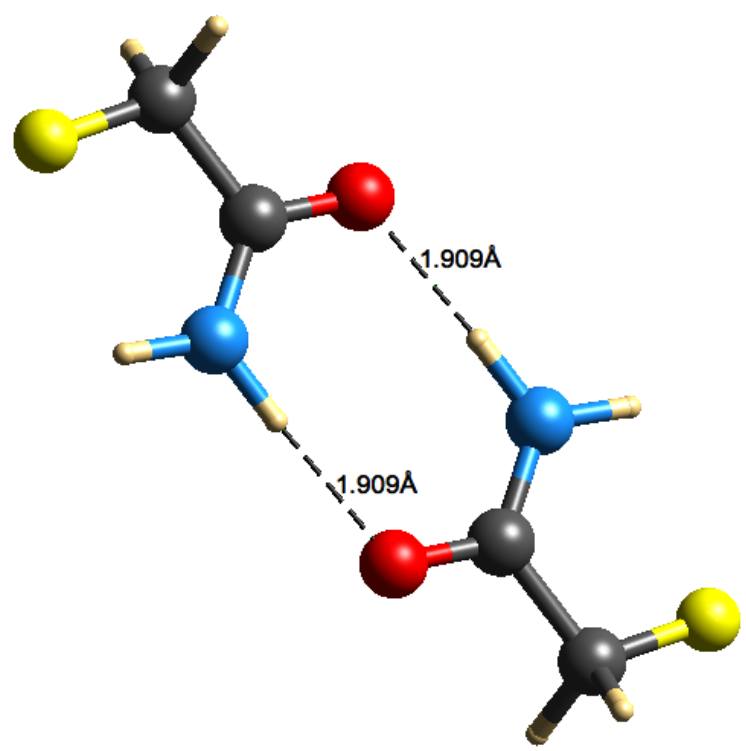

Figure S36: The molecular structure of FACETA01 ${ }^{m *}$.

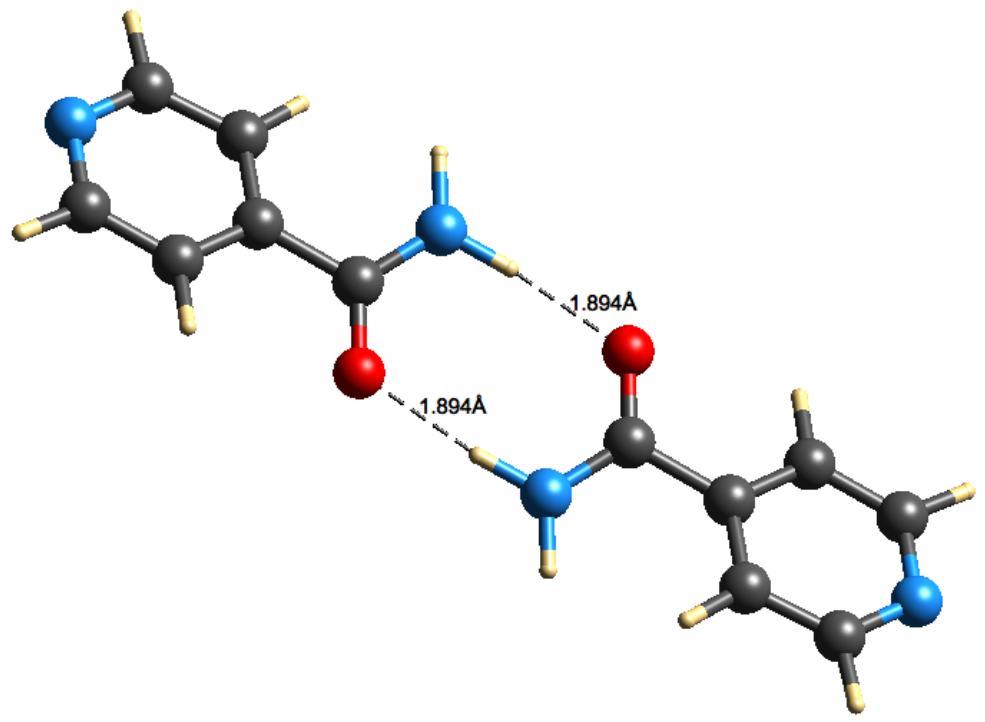

Figure S37: The molecular structure of ULAWAF0 $5^{m *}$. 


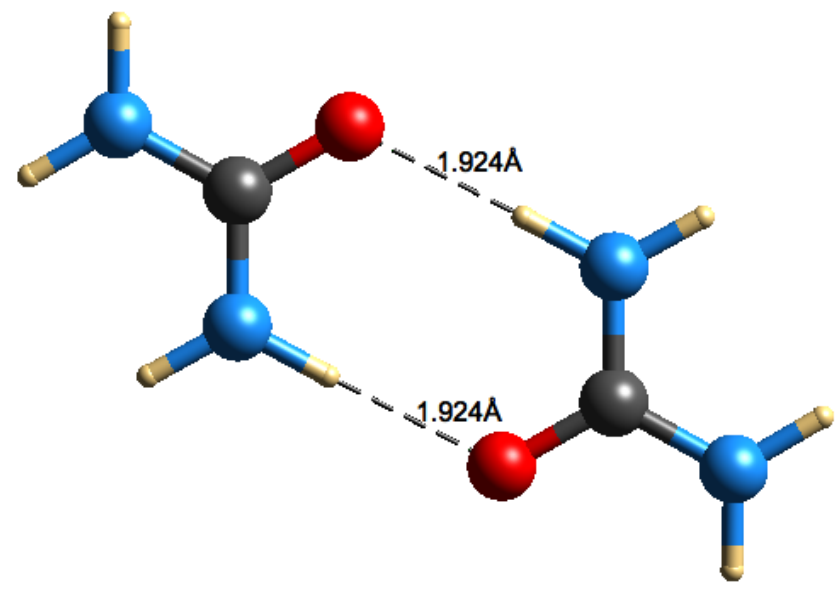

Figure S38: The molecular structure of UROXAL01 ${ }^{m *}$.

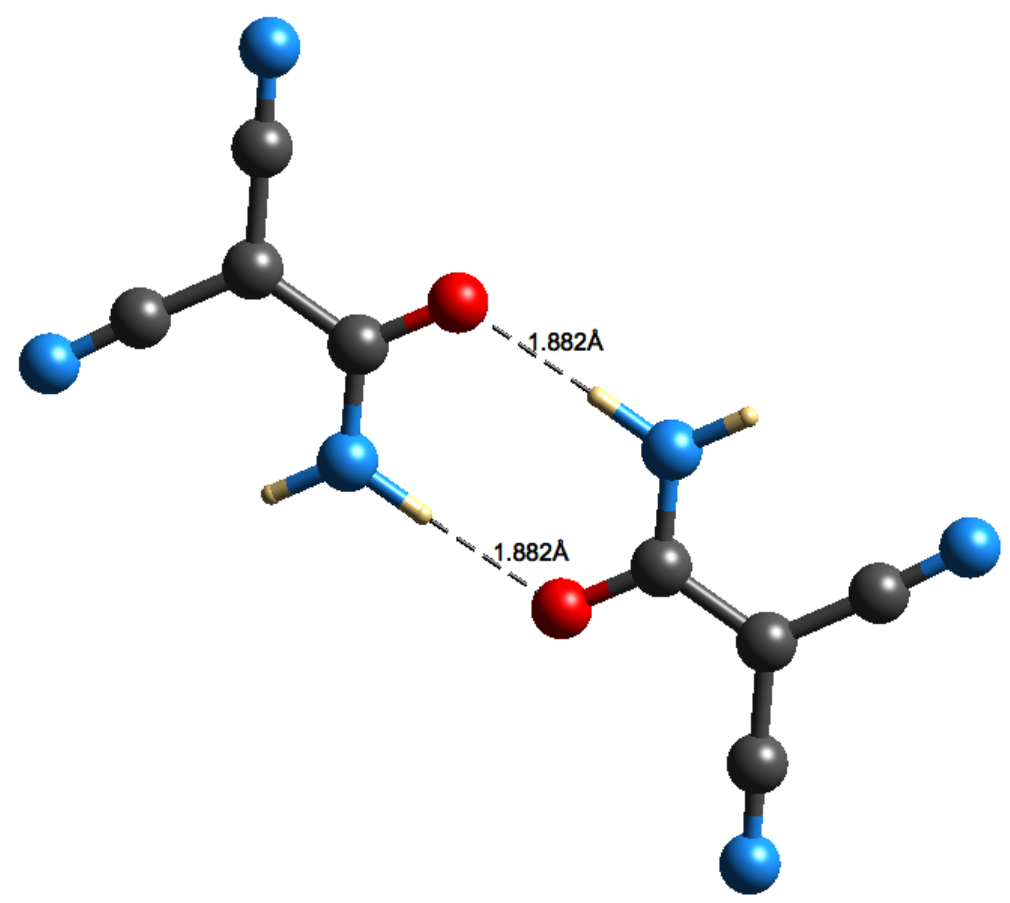

Figure S39: The molecular structure of ZECWUB01 ${ }^{m *}$. 
S8.1.6 O-H $\cdots O$ interactions

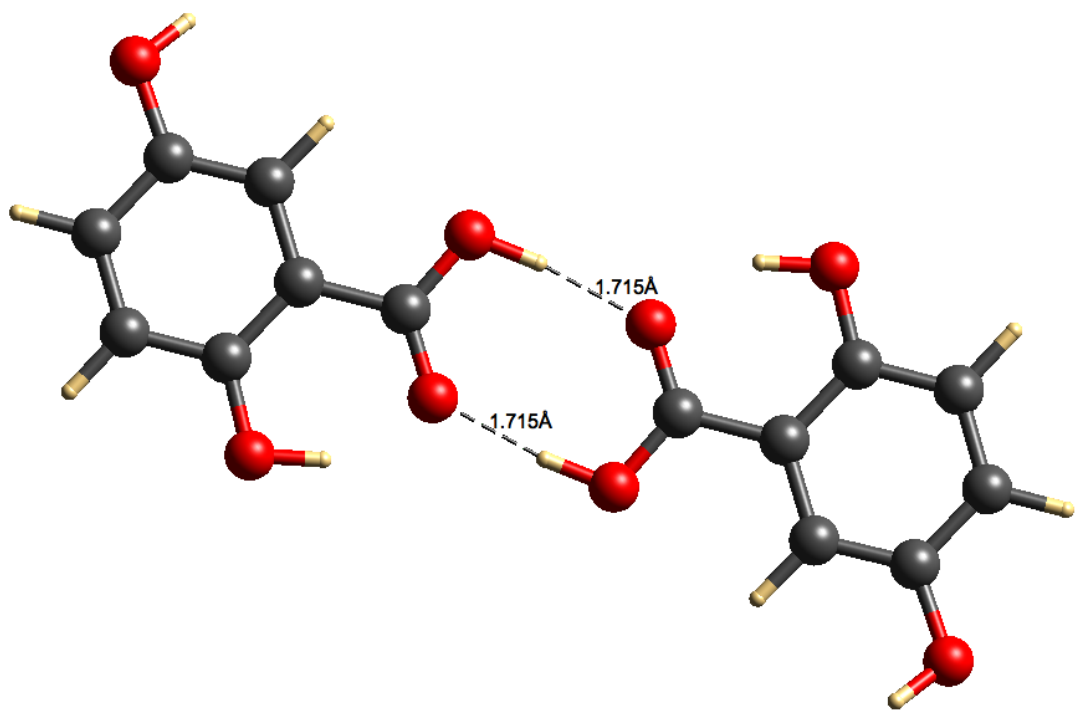

Figure S40: The molecular structure of BESKAL10 ${ }^{m *}$.

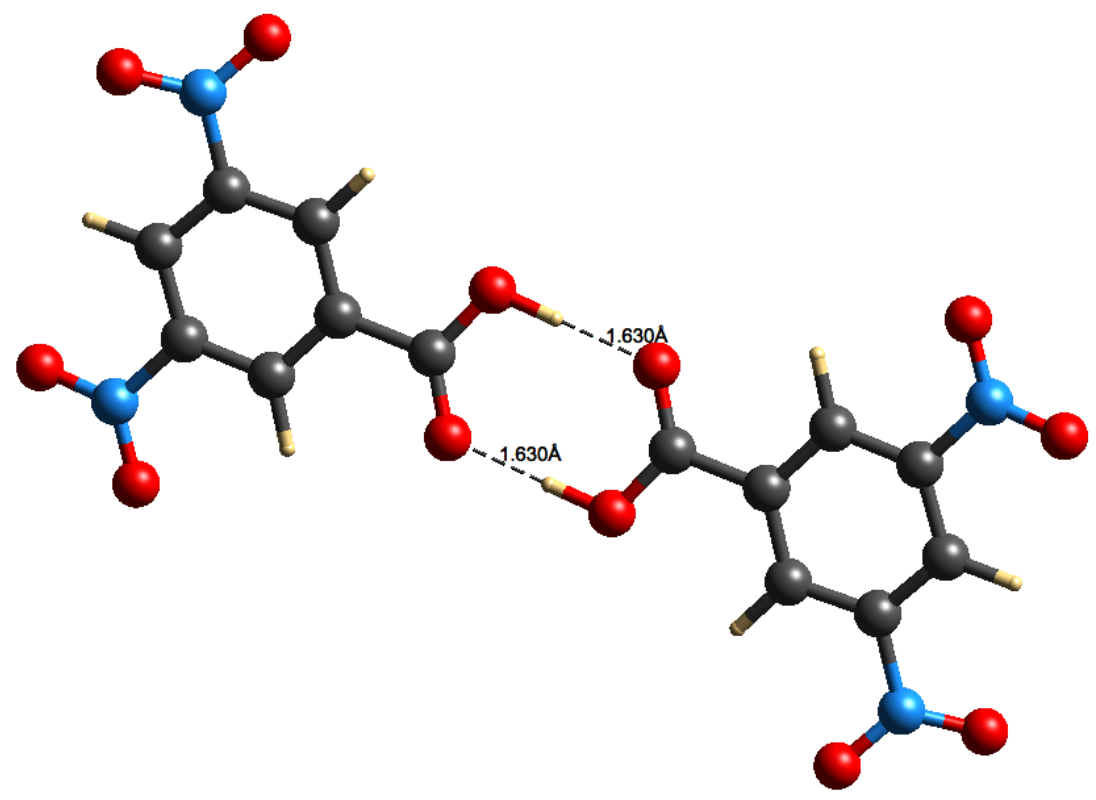

Figure S41: The molecular structure of CUKCAM18 ${ }^{m *}$. 


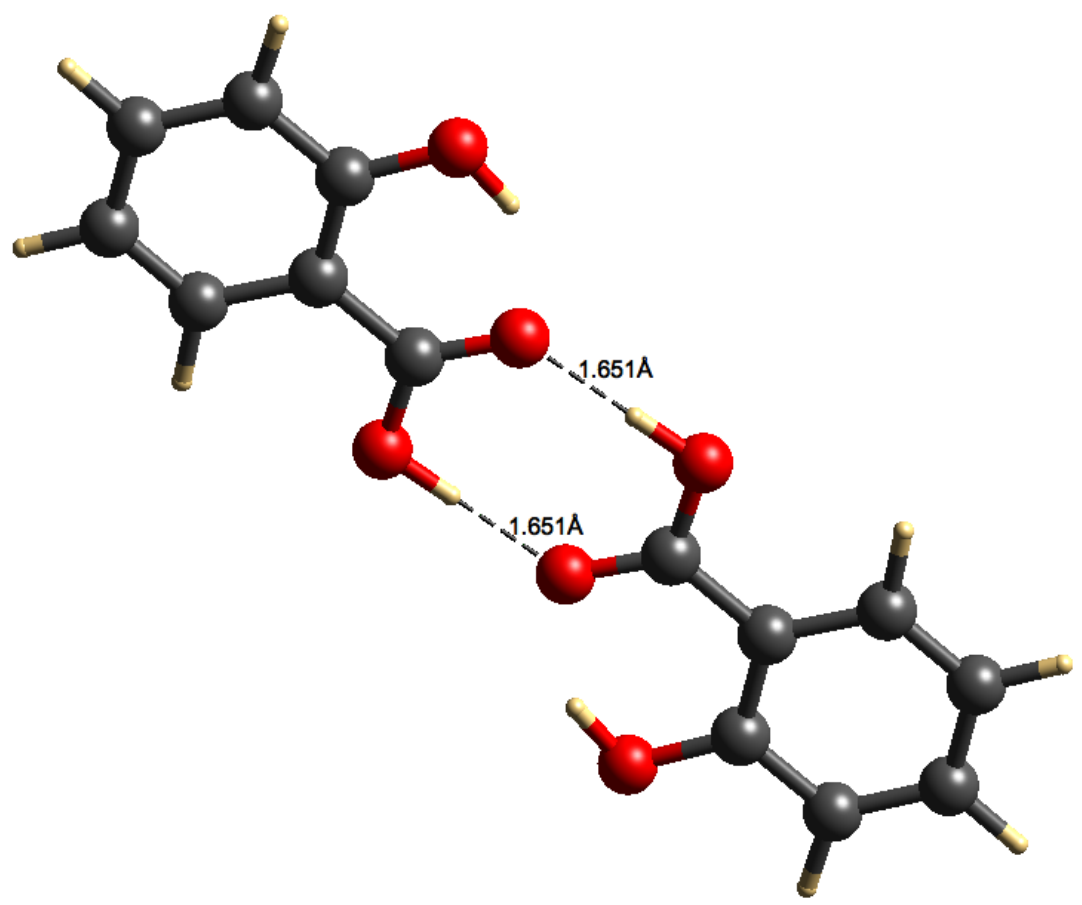

Figure S42: The molecular structure of SALIAC12 ${ }^{m *}$.

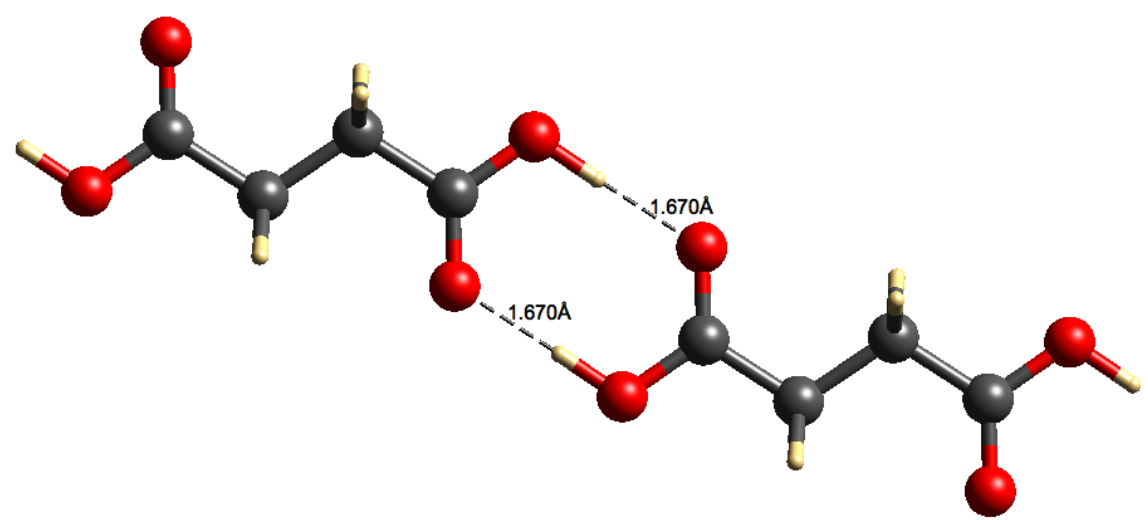

Figure S43: The molecular structure of SUCACB03 ${ }^{m *}$. 


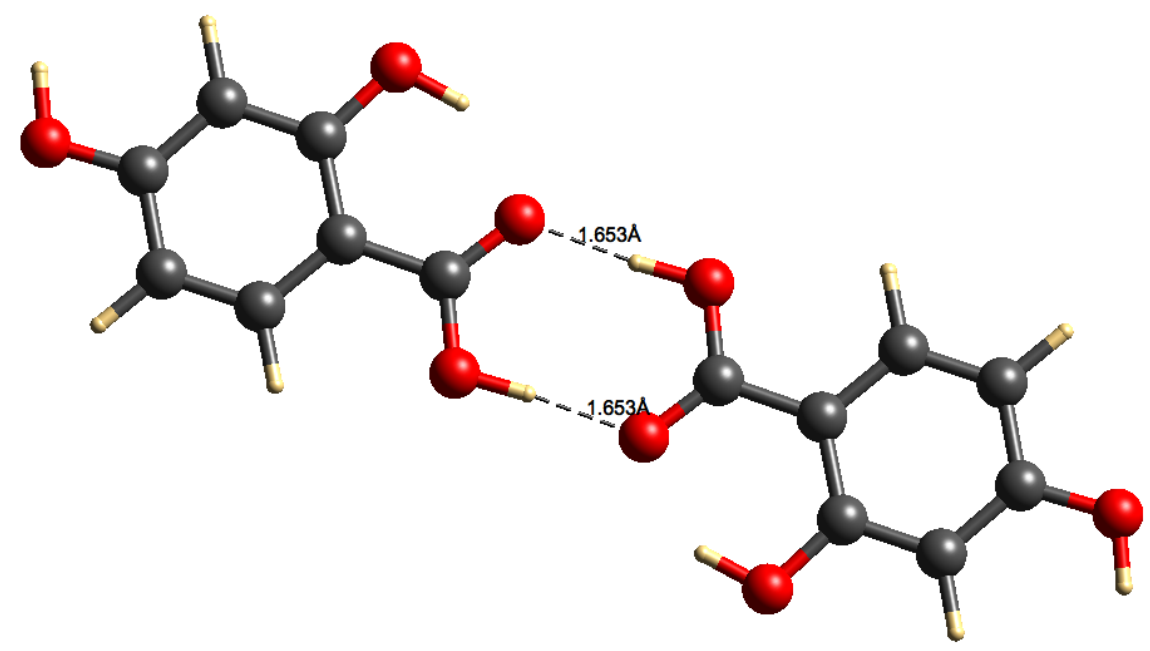

Figure S44: The molecular structure of ZZZEEU05 ${ }^{m *}$. 


\section{S8.2 Halogen bonds}

S8.2.1 $\quad \mathrm{Cl} \cdots \mathrm{N}$ interactions

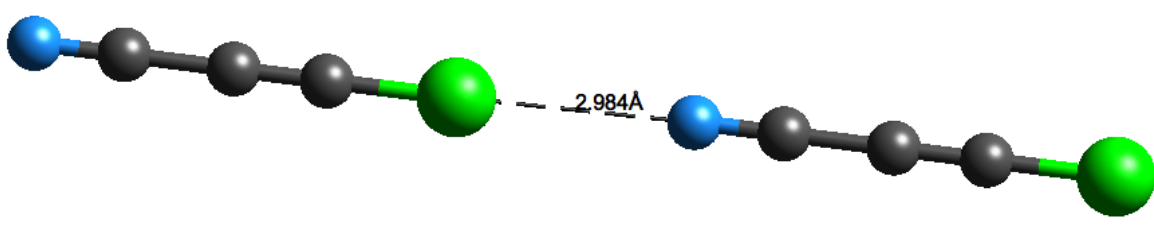

Figure S45: The molecular structure of $\mathrm{CCACENN}^{s}$.

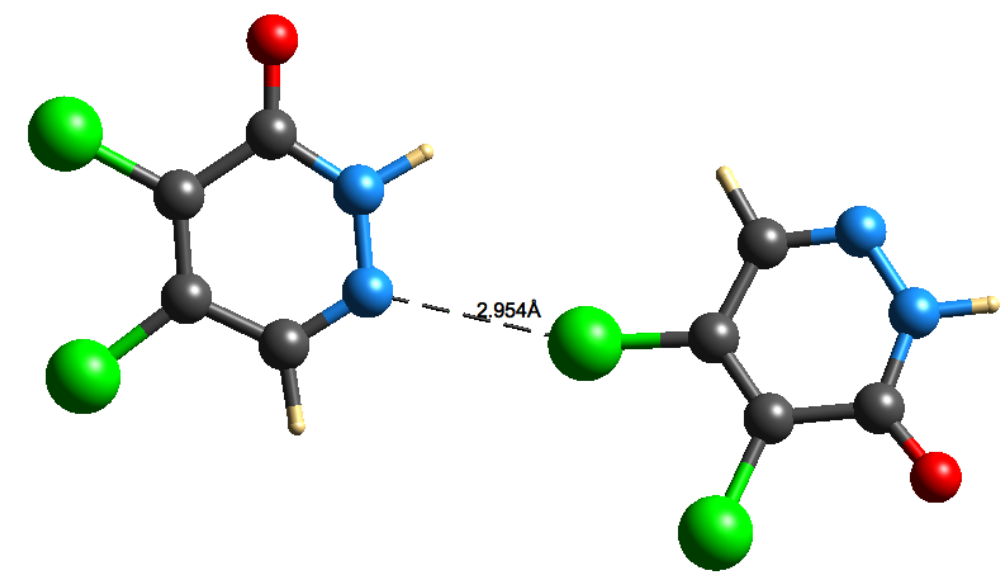

Figure S46: The molecular structure of DESKER01 ${ }^{s}$.

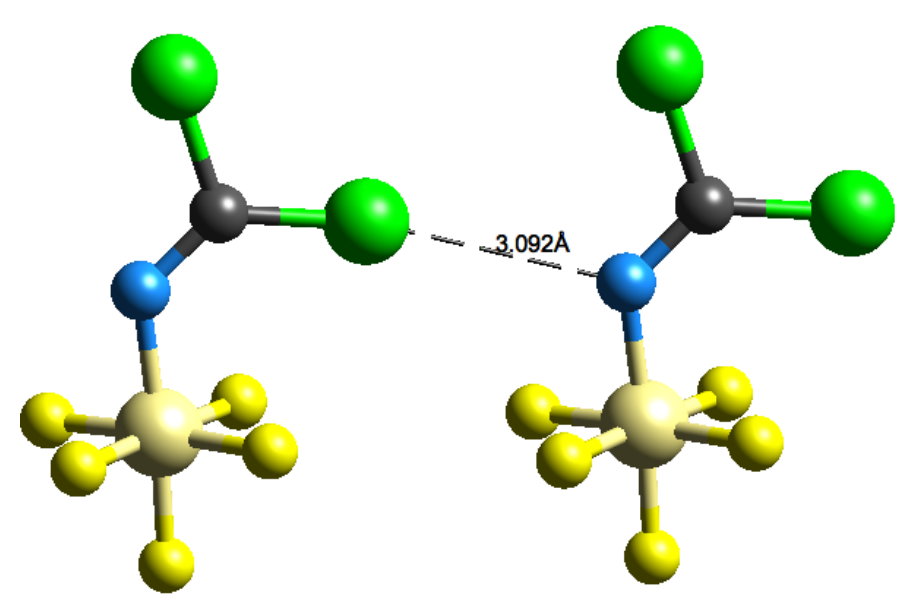

Figure S47: The molecular structure of NABZAS ${ }^{s}$. 


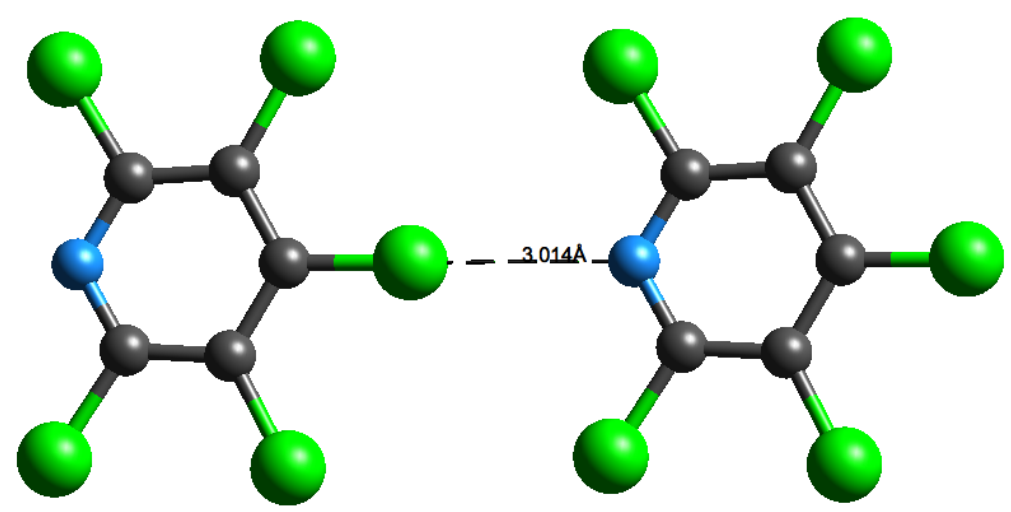

Figure S48: The molecular structure of PCLPYR ${ }^{s}$.

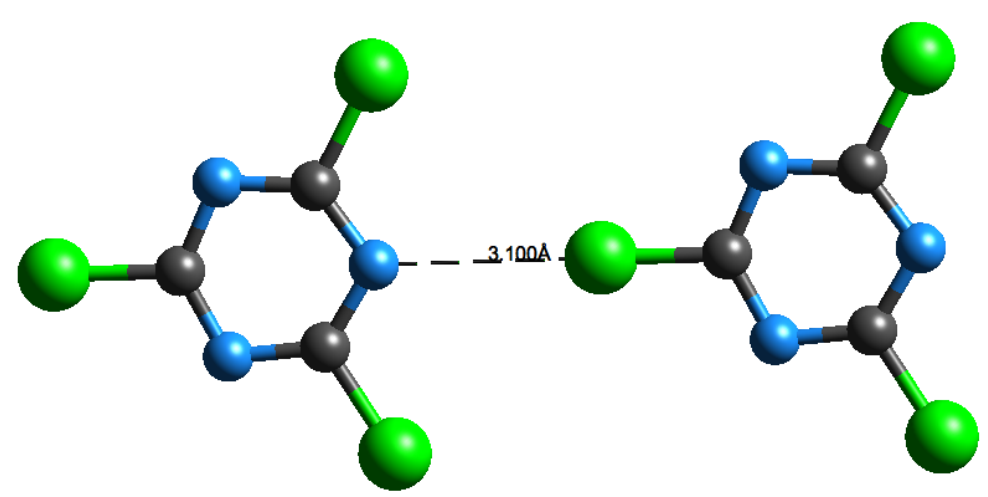

Figure S49: The molecular structure of VUGSIZ ${ }^{s}$.

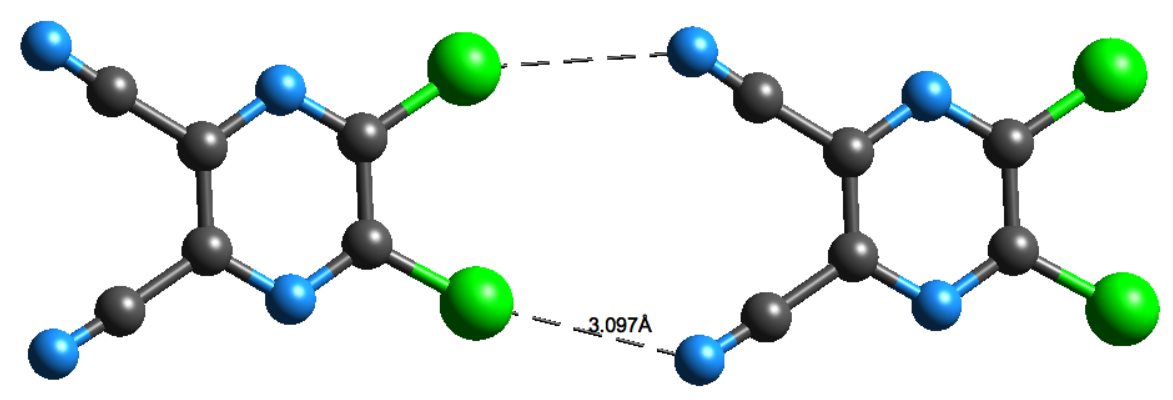

Figure S50: The molecular structure of $\mathrm{PALPAV}^{m}$. 


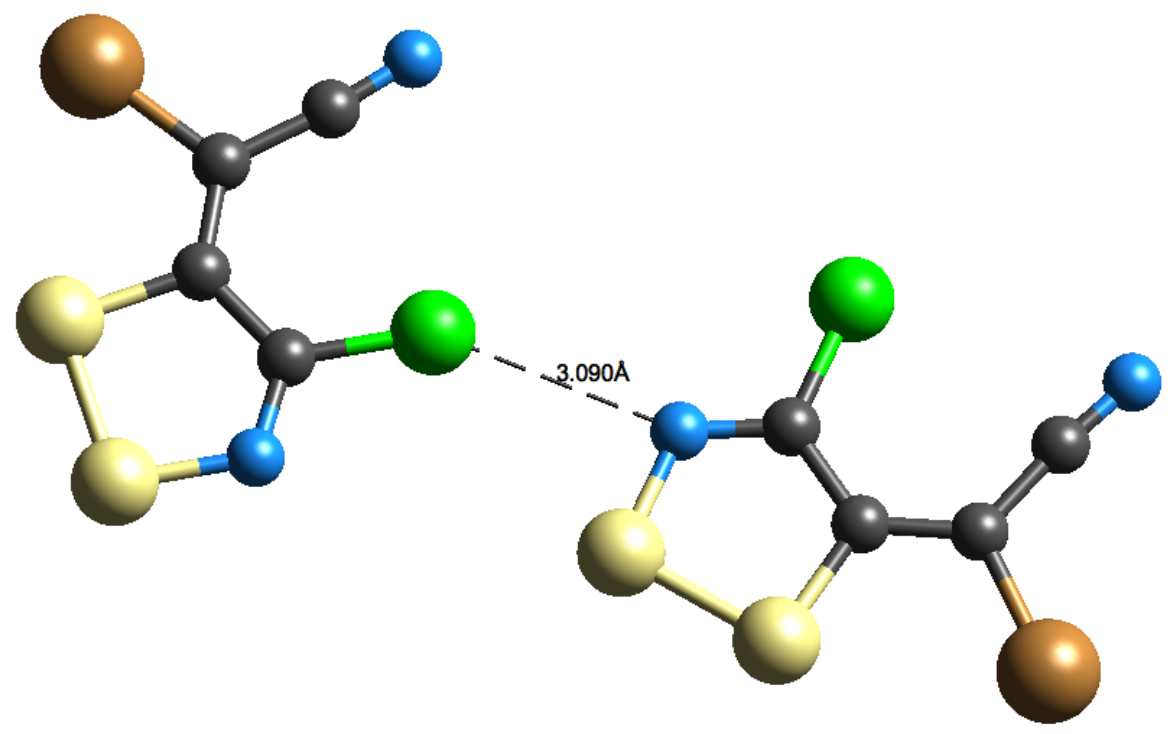

Figure S51: The molecular structure of XIZPON ${ }^{s}$. 
S8.2.2 $\mathrm{Cl} \cdots \mathrm{O}$ interactions

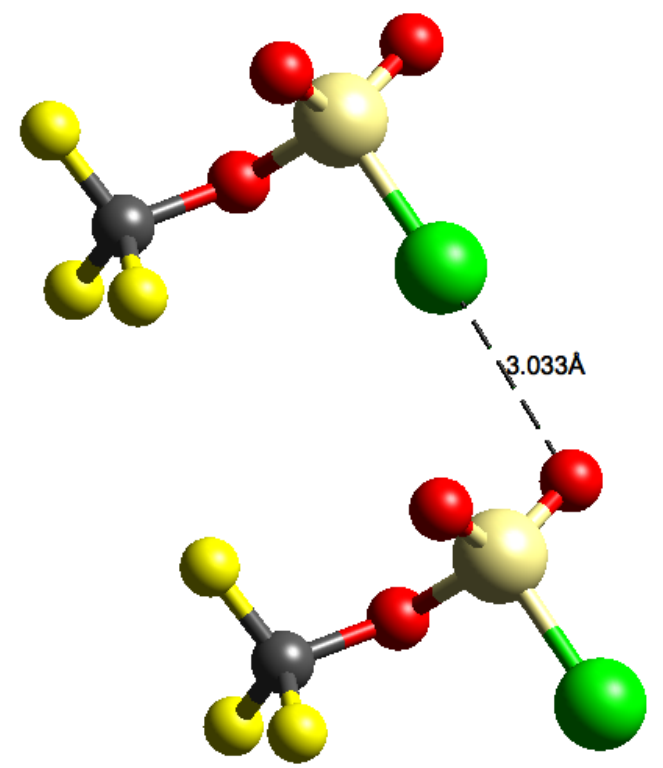

Figure S52: The molecular structure of BEDMON ${ }^{s}$.

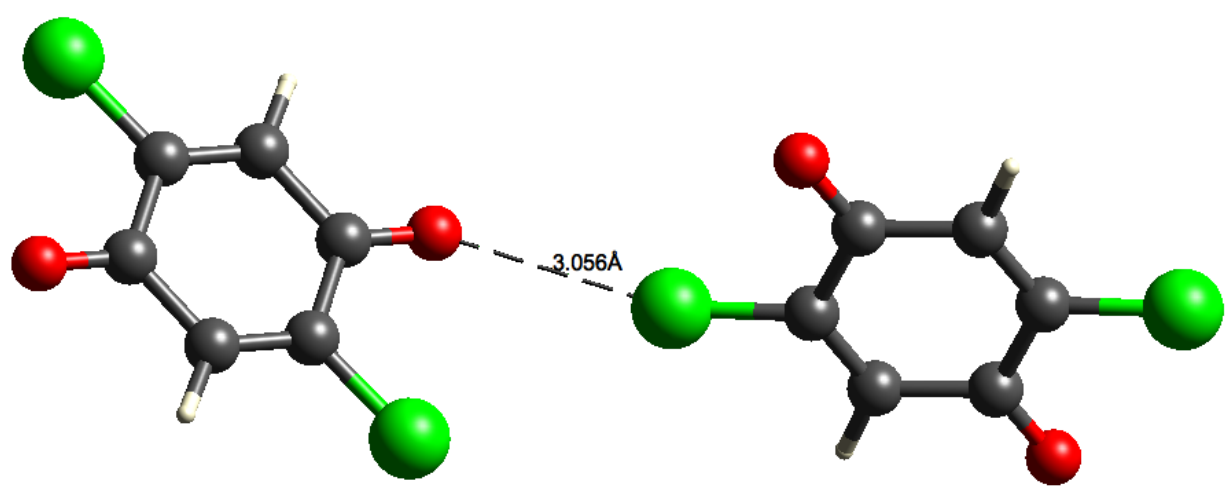

Figure S53: The molecular structure of BZQDCL11 ${ }^{s}$.

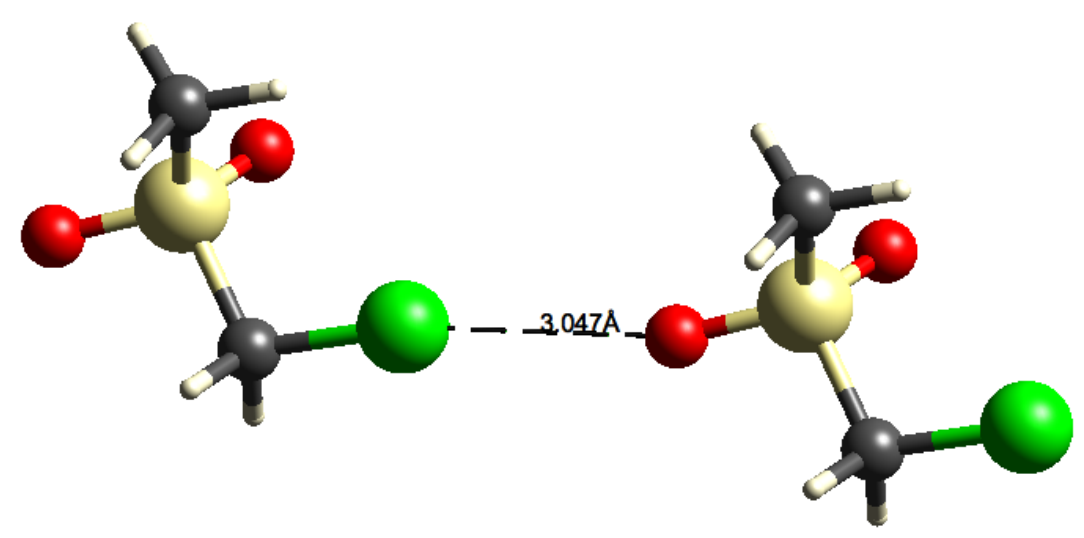

Figure S54: The molecular structure of CORDUI ${ }^{s}$. 


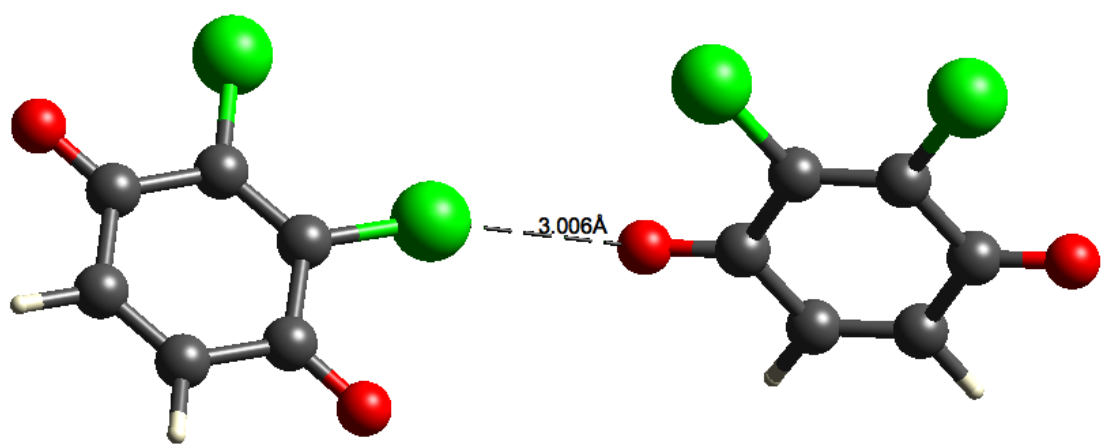

Figure S55: The molecular structure of DCLBZQ20s.

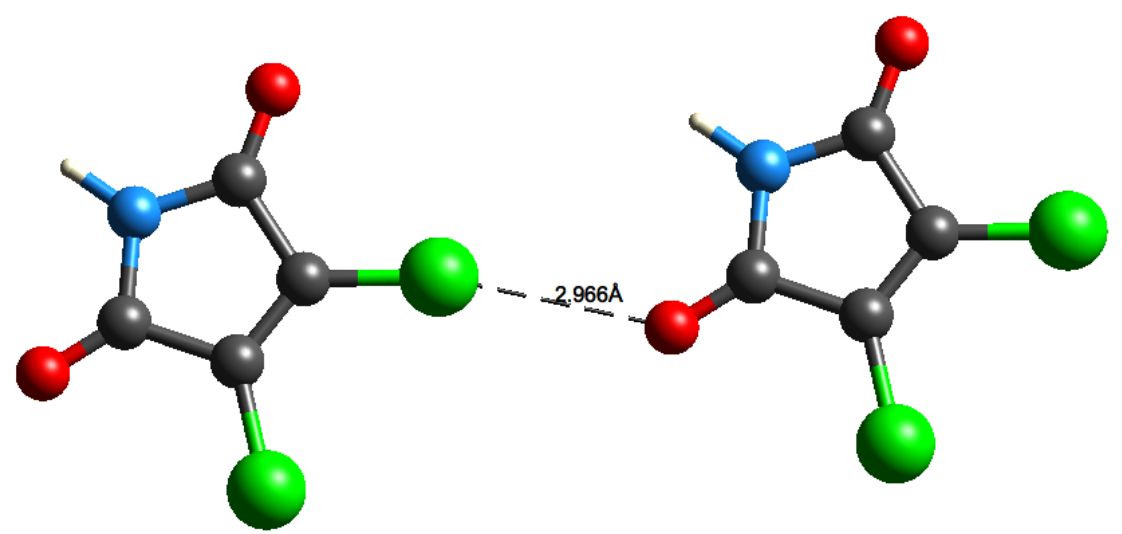

Figure S56: The molecular structure of IRUFEH01 ${ }^{s}$.

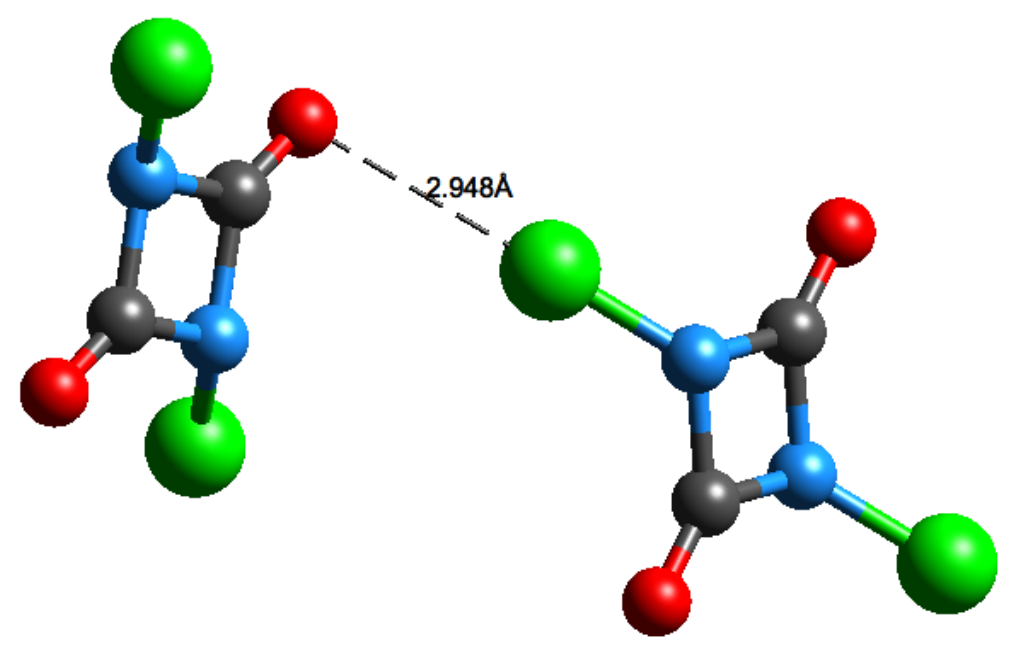

Figure S57: The molecular structure of JOJTIL ${ }^{s}$. 


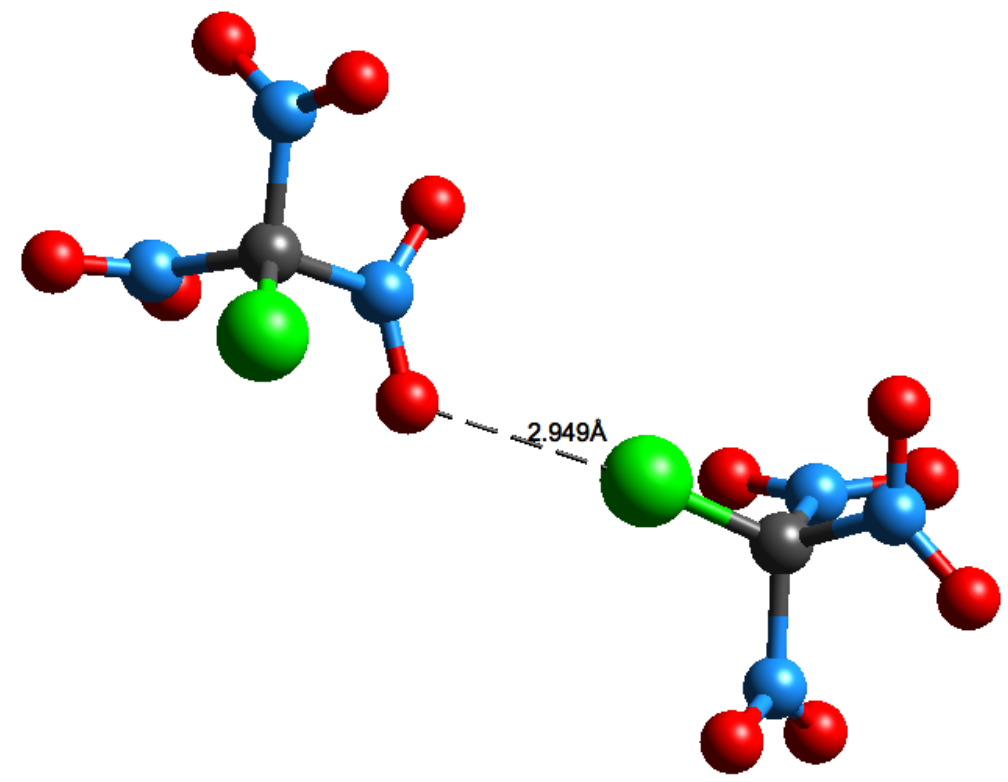

Figure S58: The molecular structure of RUBSUD ${ }^{s}$.

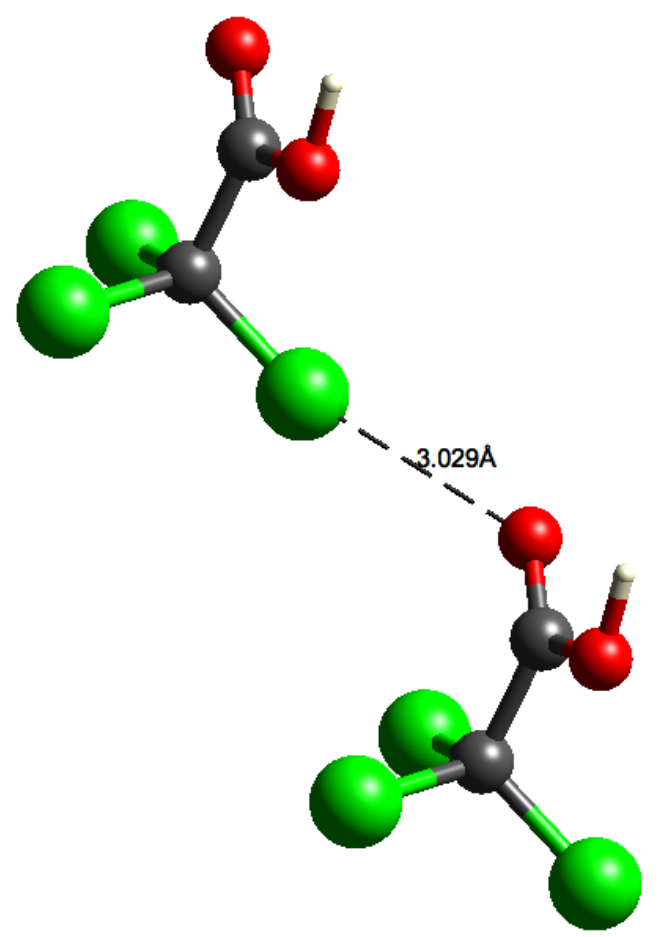

Figure S59: The molecular structure of TCACAD01 ${ }^{s}$. 


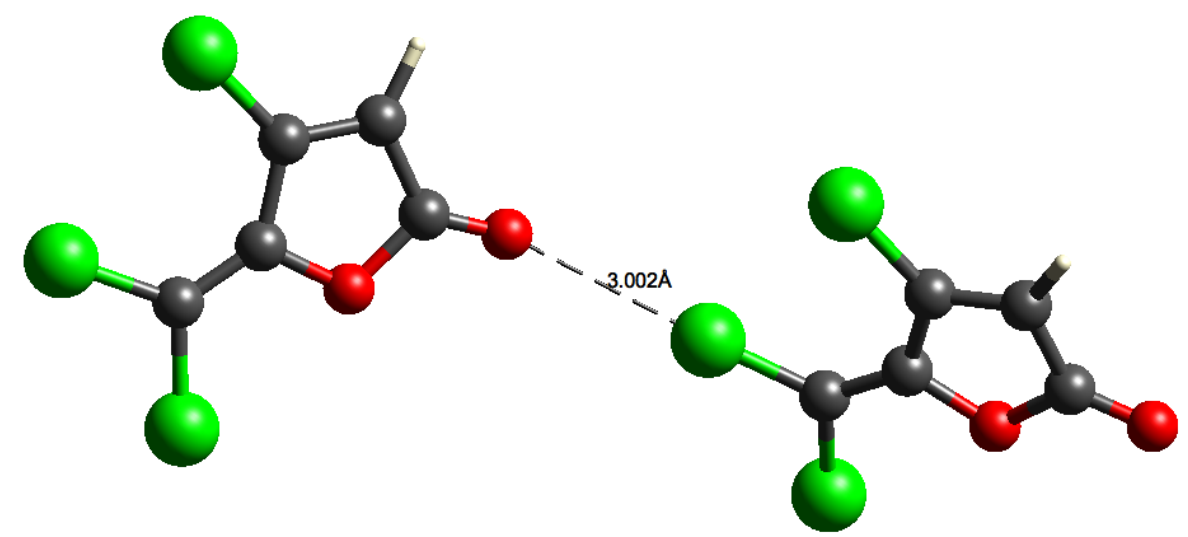

Figure S60: The molecular structure of GEXWUB ${ }^{s}$.

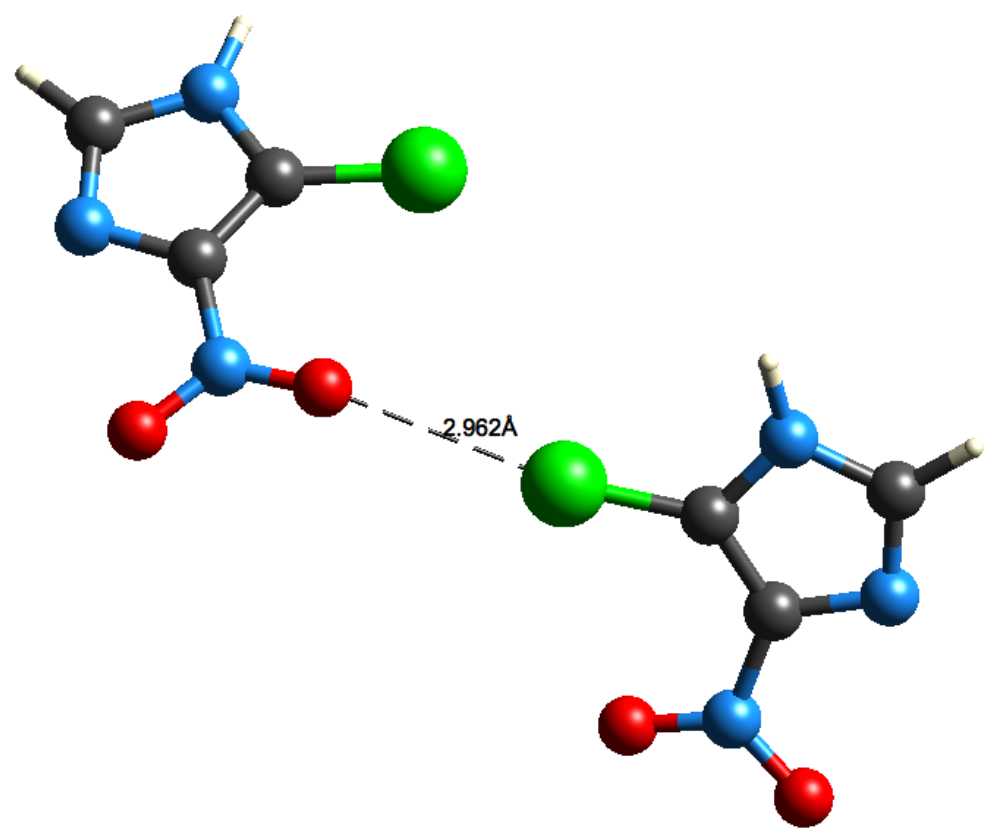

Figure S61: The molecular structure of PEPFUL ${ }^{s}$. 
S8.2.3 $\quad \mathrm{Br} \cdots \mathrm{N}$ interactions

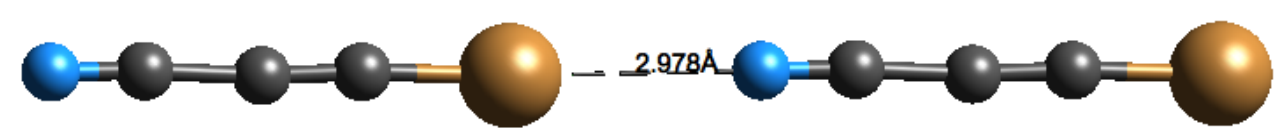

Figure S62: The molecular structure of BCACENN ${ }^{s}$.

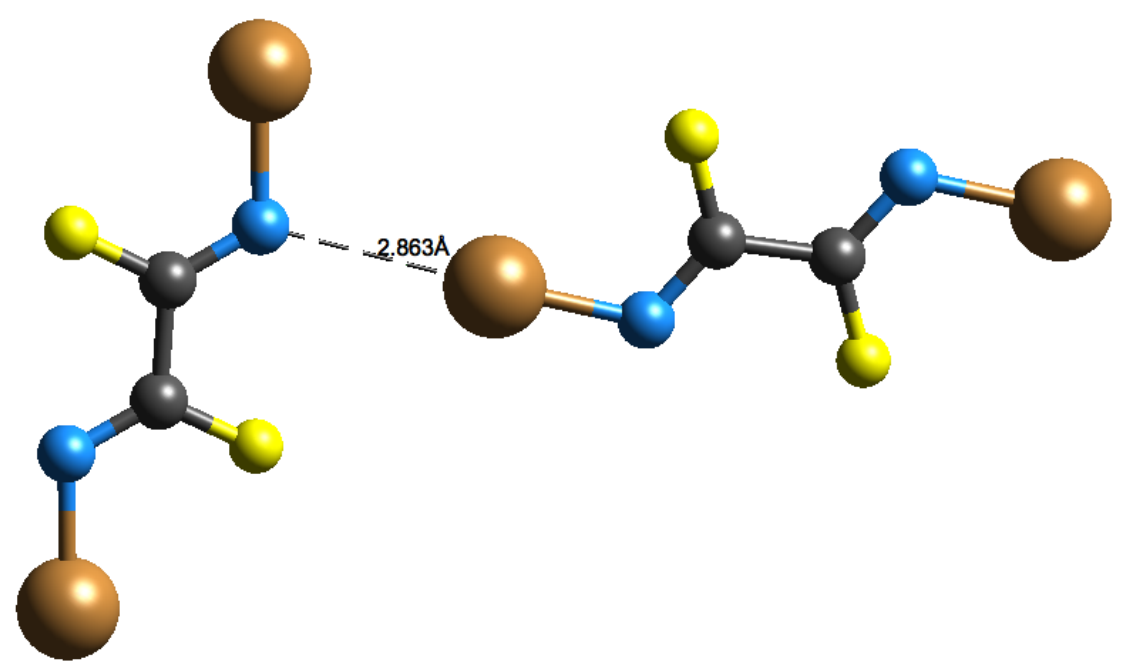

Figure S63: The molecular structure of BONFIT ${ }^{s}$.

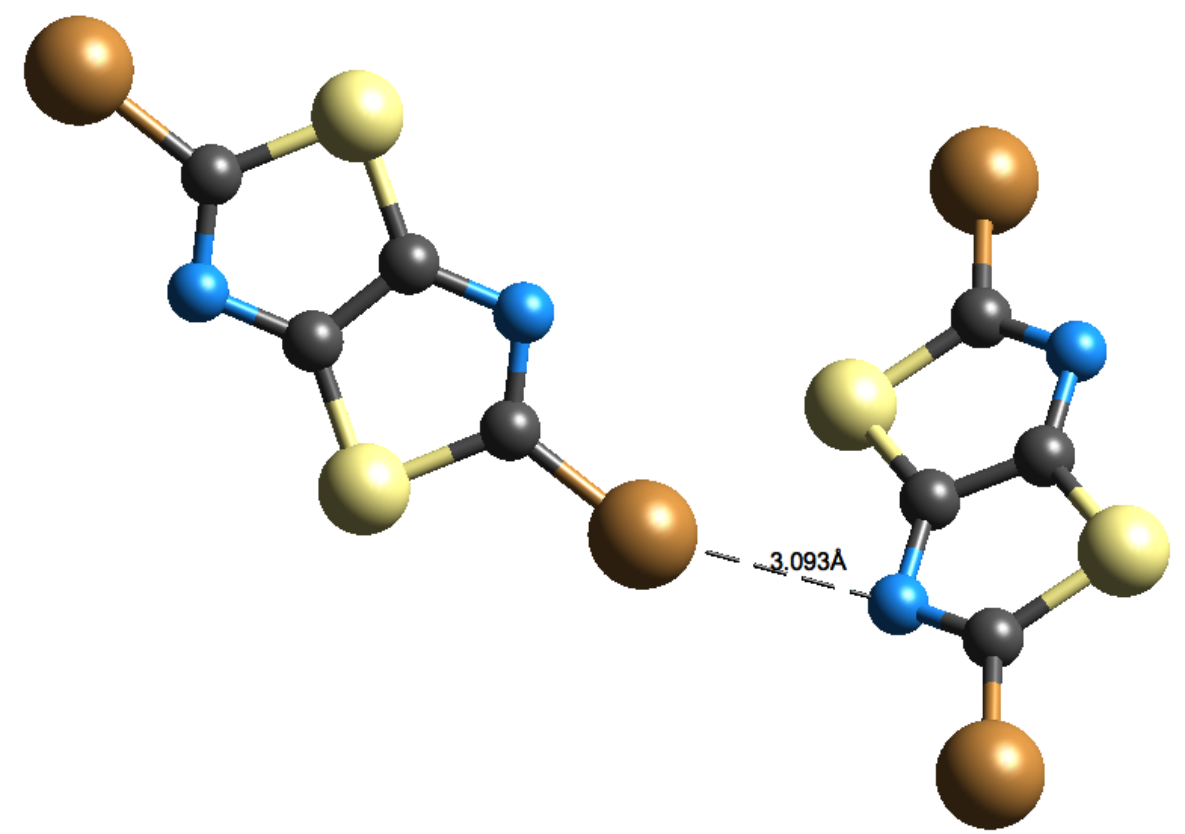

Figure S64: The molecular structure of QONHUX ${ }^{s}$. 


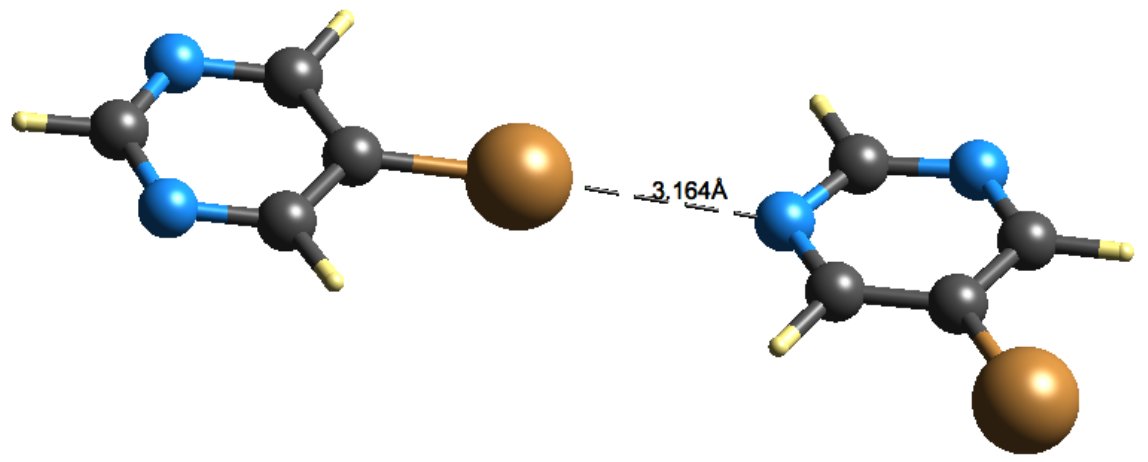

Figure S65: The molecular structure of RIRFOON ${ }^{s}$.

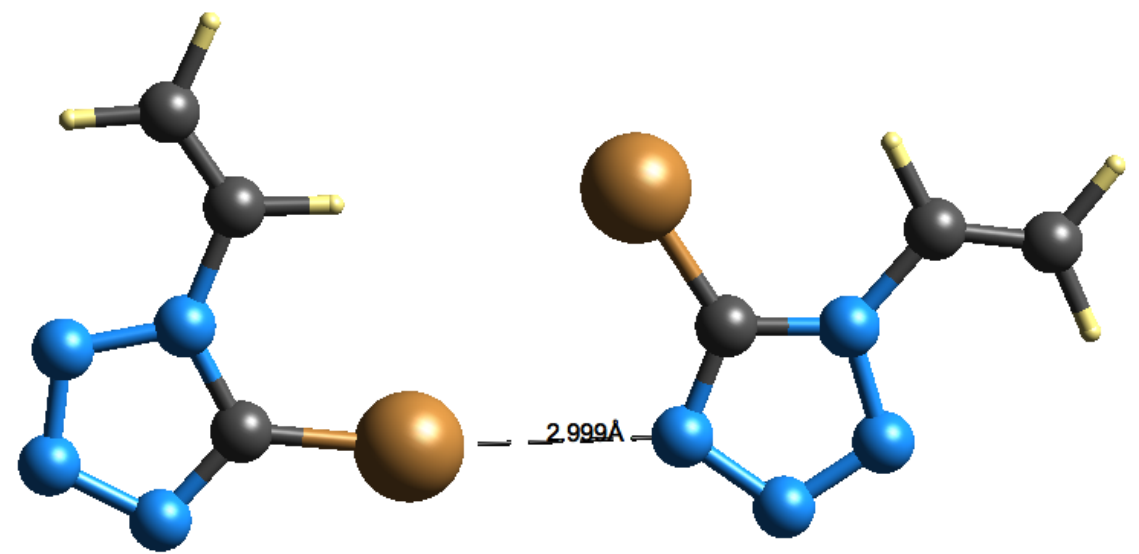

Figure S66: The molecular structure of KUYCUD ${ }^{s}$. 
S8.2.4 $\mathrm{Br} \cdots \mathrm{O}$ interactions

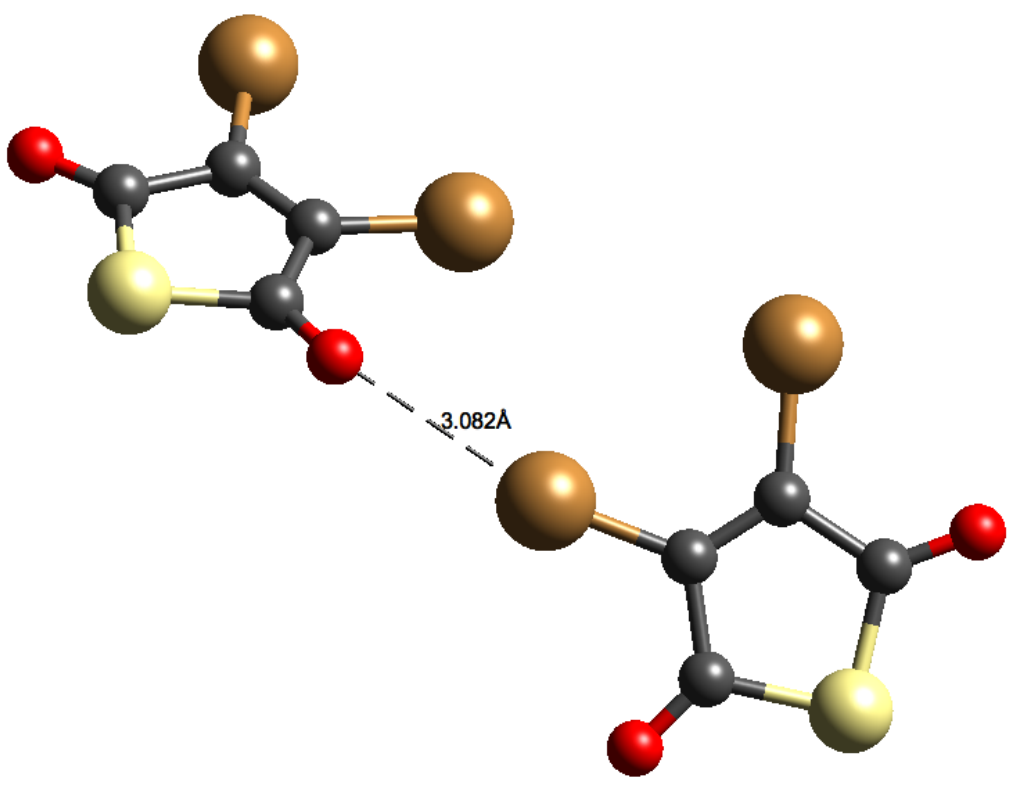

Figure S67: The molecular structure of BMLTAAN $^{s}$.

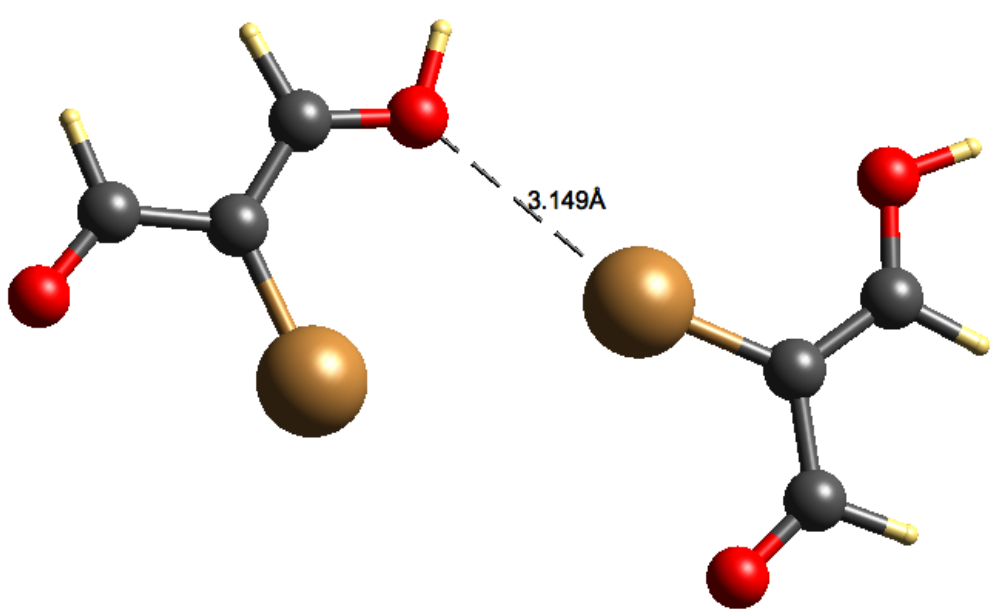

Figure S68: The molecular structure of $\mathrm{CIRSONN}^{s}$. 


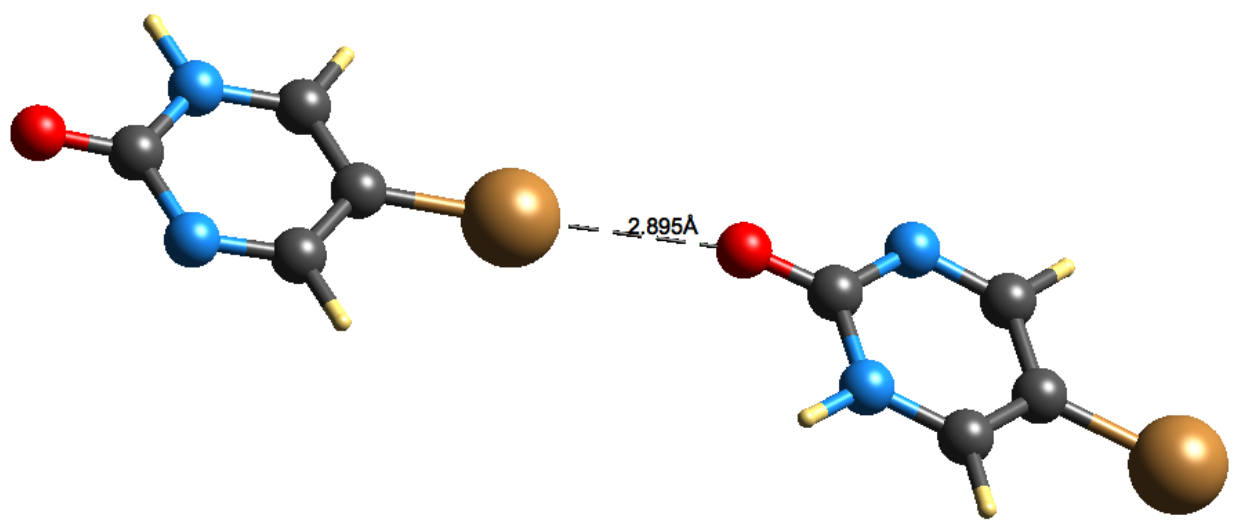

Figure S69: The molecular structure of JEVVOW ${ }^{s}$.

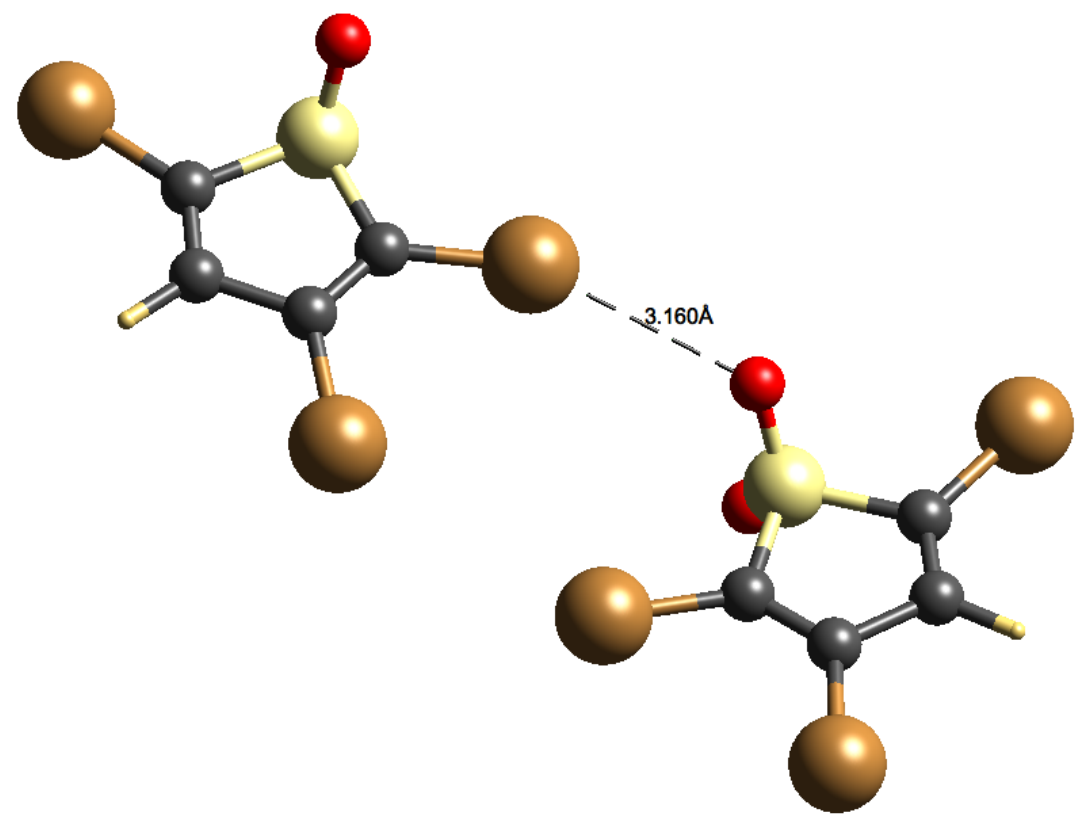

Figure S70: The molecular structure of VAQXUG ${ }^{s}$.

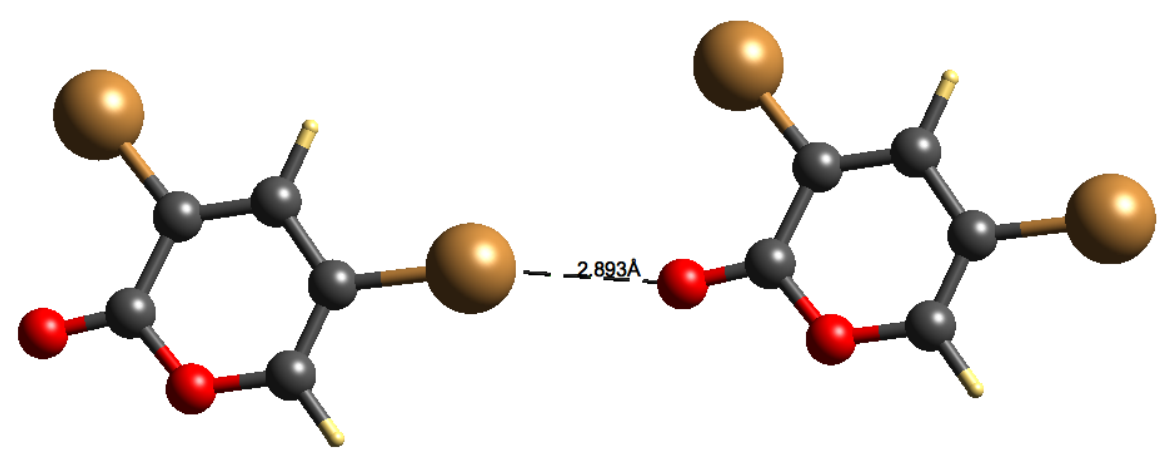

Figure S71: The molecular structure of VEWTAU ${ }^{s}$. 


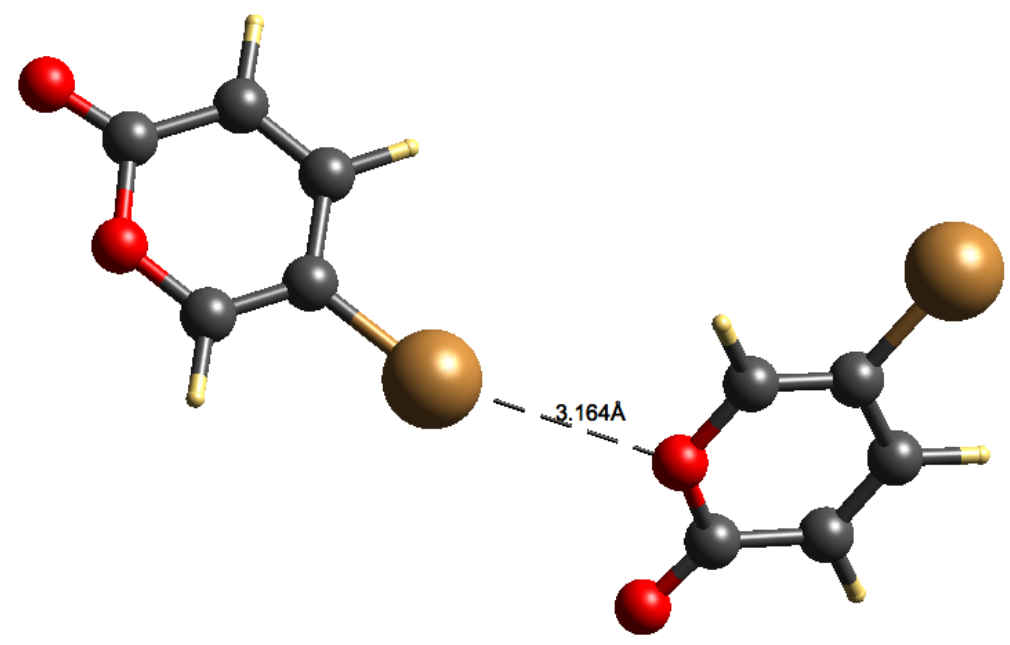

Figure S72: The molecular structure of VEWTEY ${ }^{s}$.

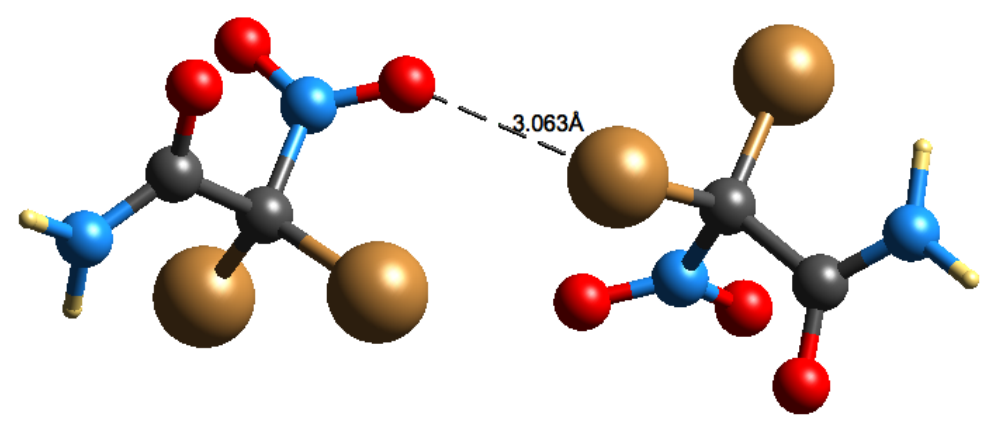

Figure S73: The molecular structure of VITVEZ ${ }^{s}$.

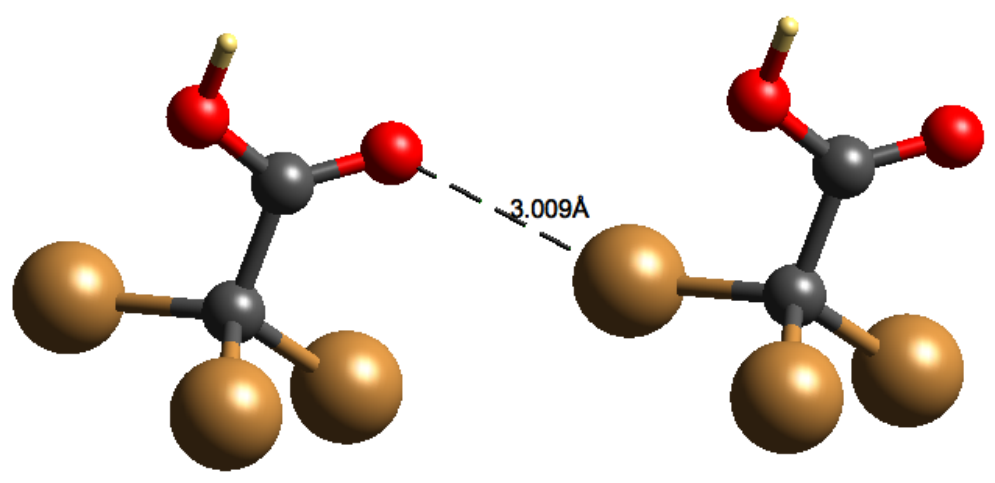

Figure S74: The molecular structure of WADFIR ${ }^{s}$. 


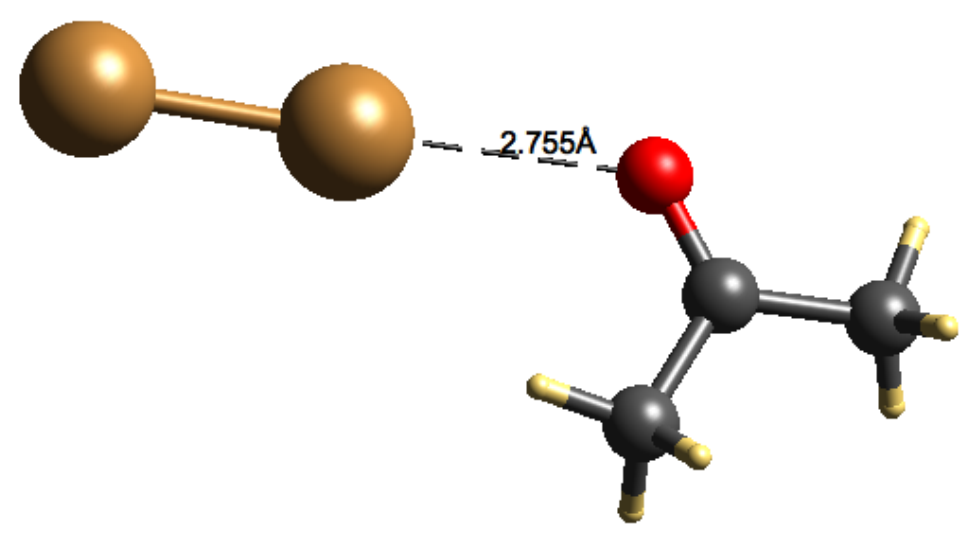

Figure S75: The molecular structure of ACETBR02 ${ }^{s}$. 


\section{S8.3 Chalcogen bonds}

S8.3.1 $\mathrm{S} \cdots \mathrm{N}$ interactions

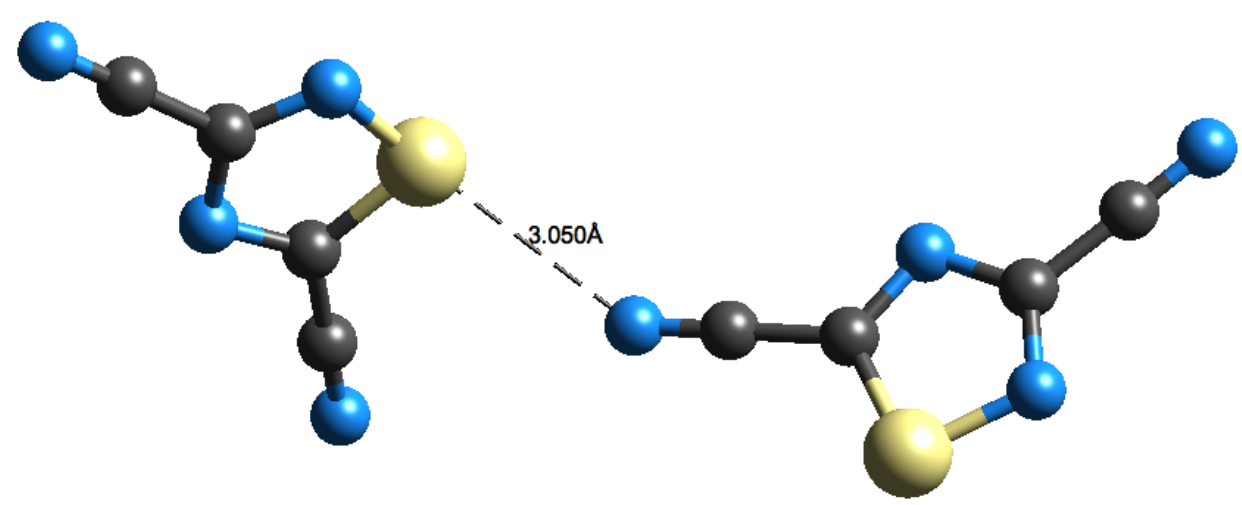

Figure S76: The molecular structure of CEBYUD ${ }^{s}$.

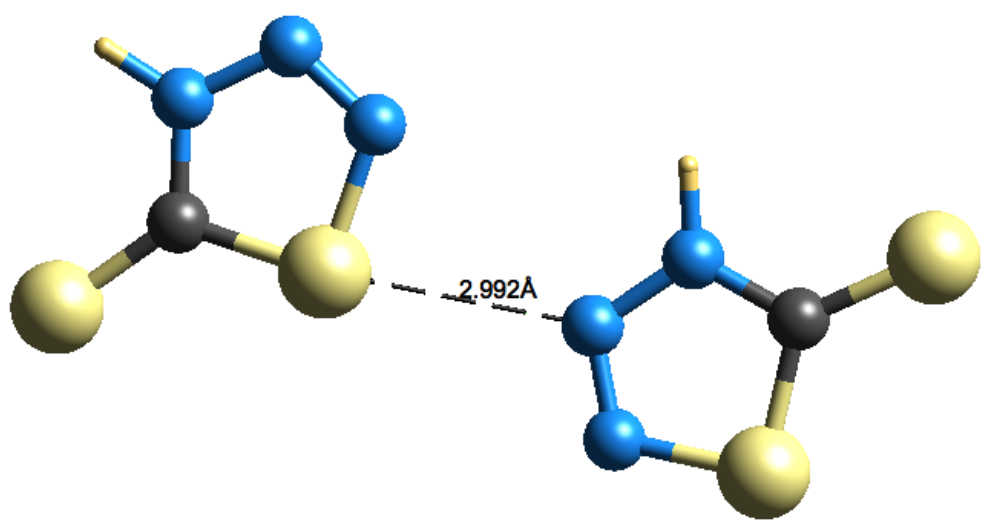

Figure S77: The molecular structure of QOBFUI ${ }^{s}$.

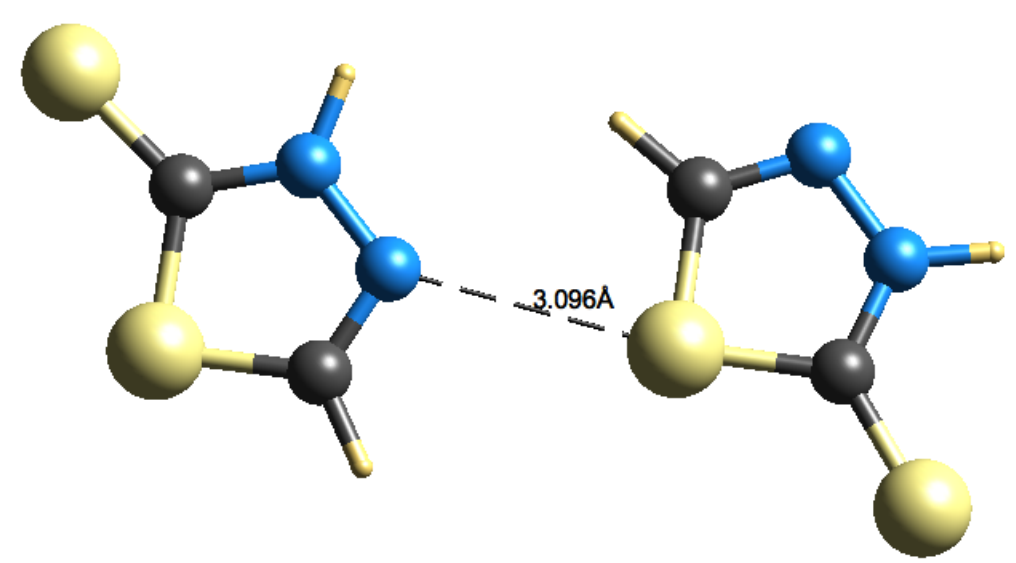

Figure S78: The molecular structure of SAZCEC ${ }^{s}$. 


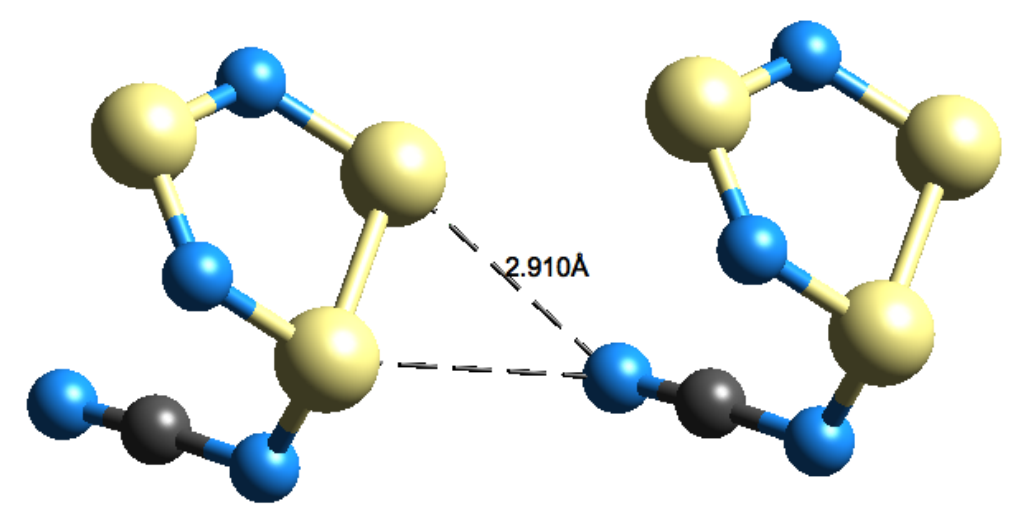

Figure S79: The molecular structure of GEDHAY ${ }^{m}$.

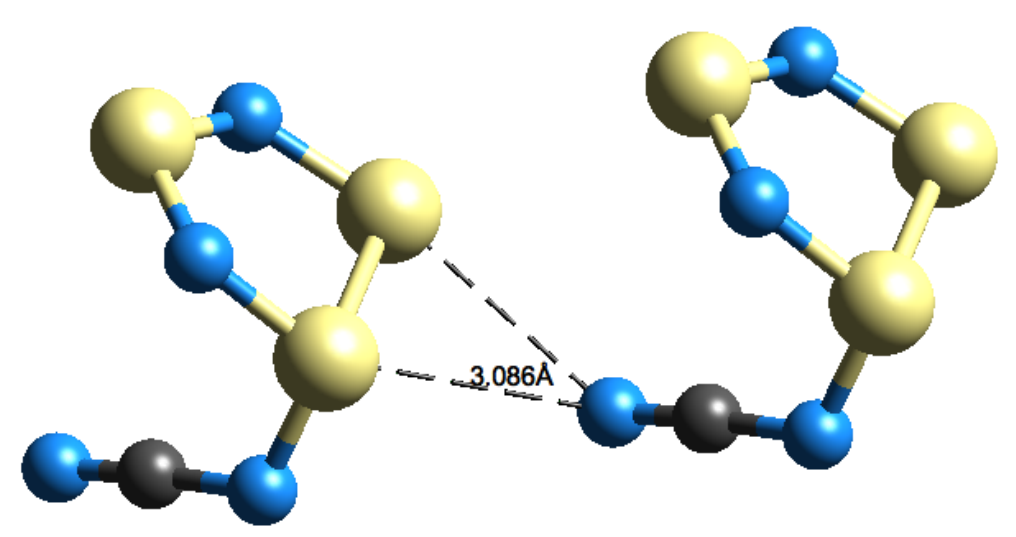

Figure S80: The molecular structure of GEDHAY ${ }^{m}$.

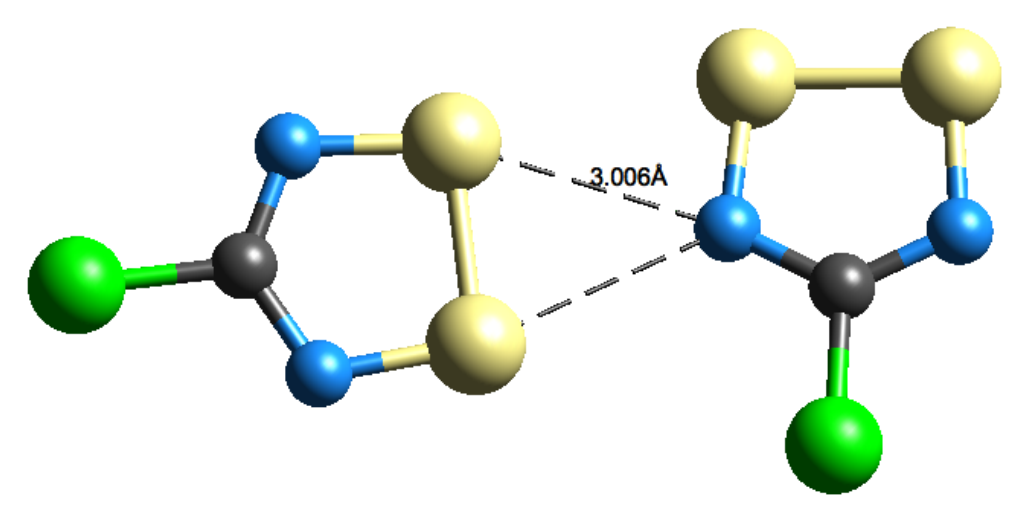

Figure S81: The molecular structure of IFULUQ04 ${ }^{m}$. 


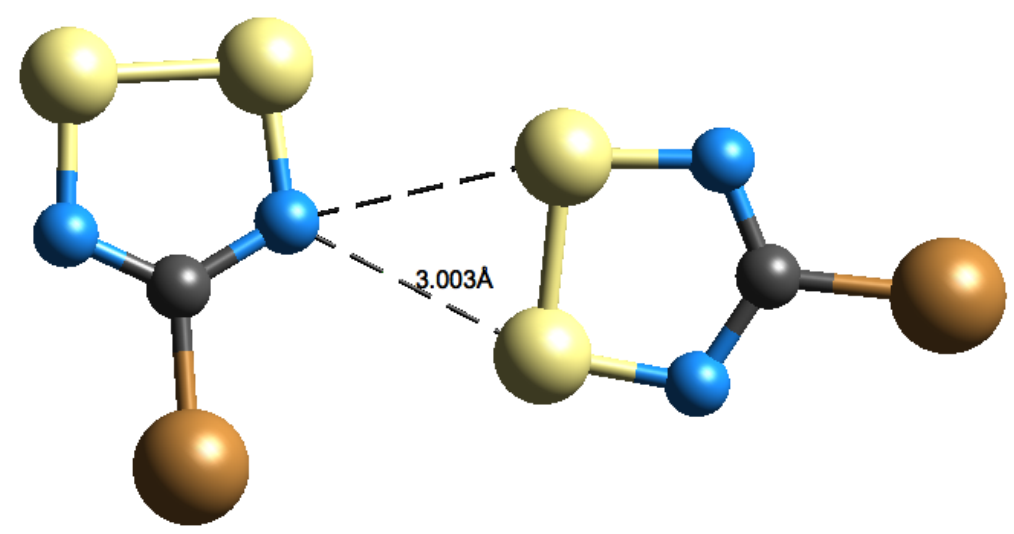

Figure S82: The molecular structure of $\mathrm{WASHEE}^{m}$.

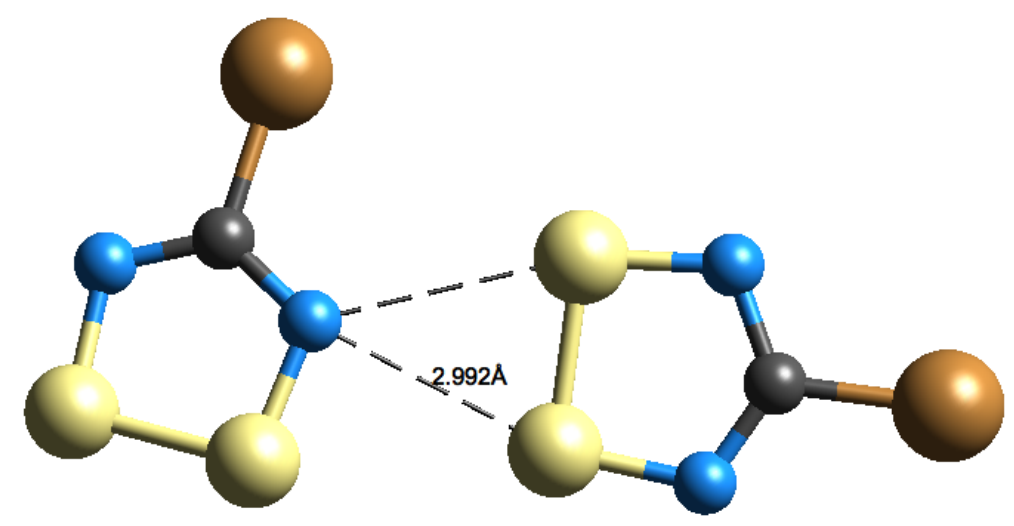

Figure S83: The molecular structure of $\mathrm{WASHEE}^{m}$.

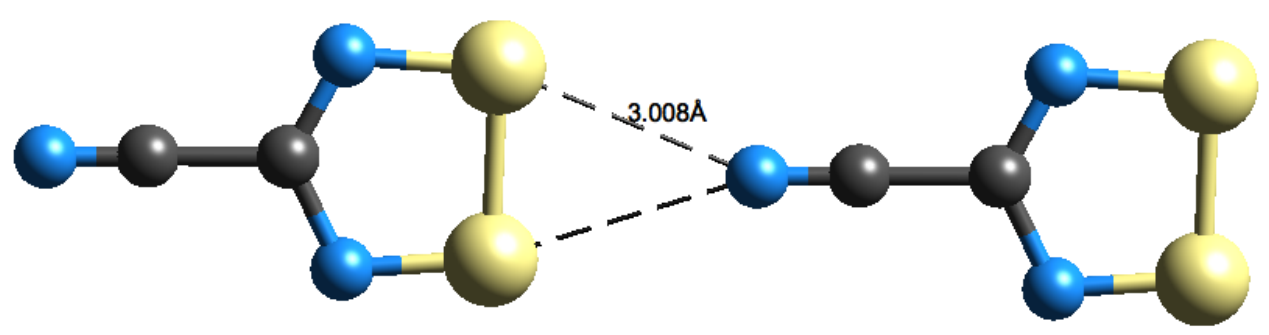

Figure S84: The molecular structure of $\mathrm{WUXPAG}^{m}$. 


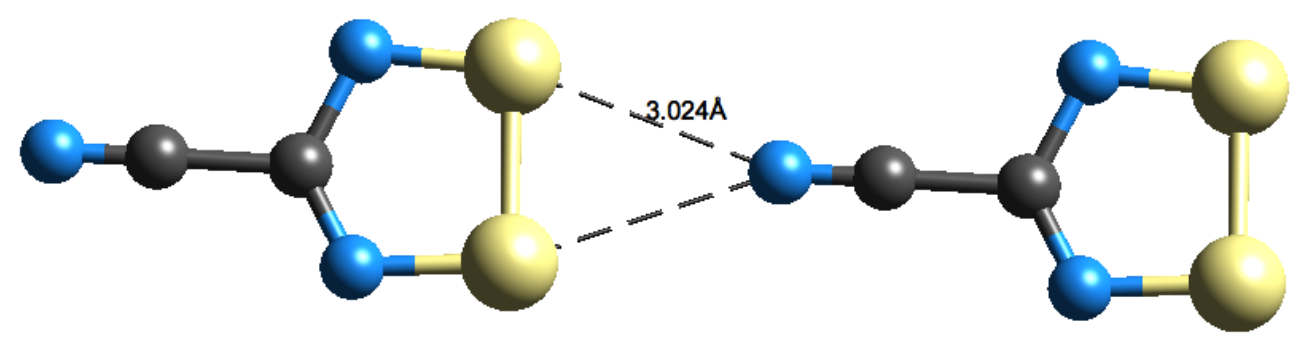

Figure S85: The molecular structure of $\mathrm{WUXPAG}^{m}$.

50 


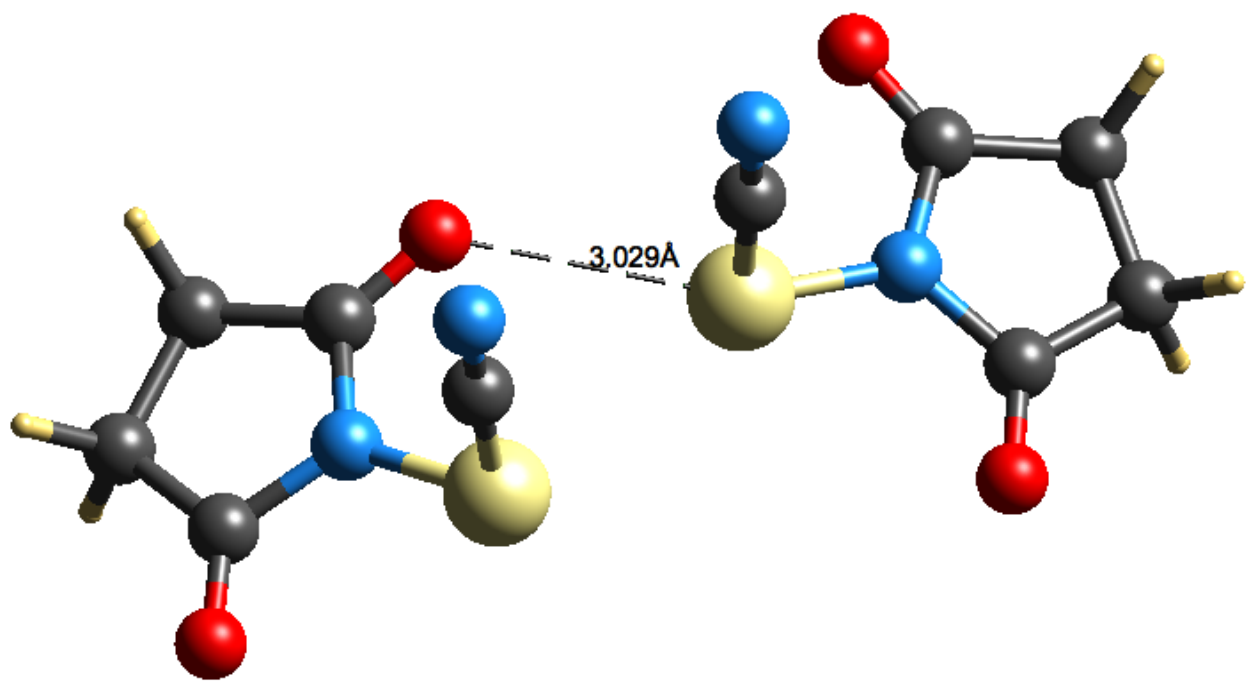

Figure S86: The molecular structure of PAFVEY ${ }^{s}$.

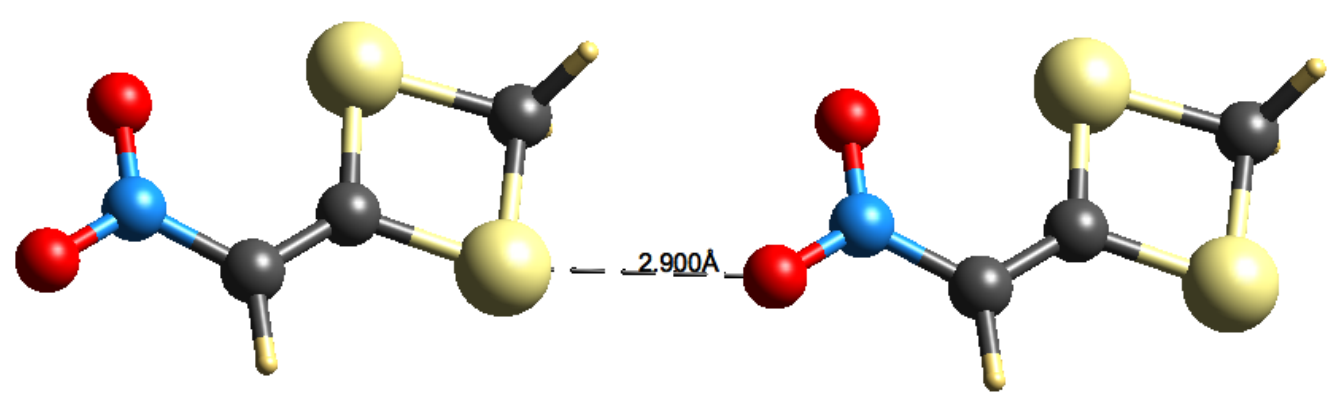

Figure S87: The molecular structure of WOCQEK ${ }^{s}$. 


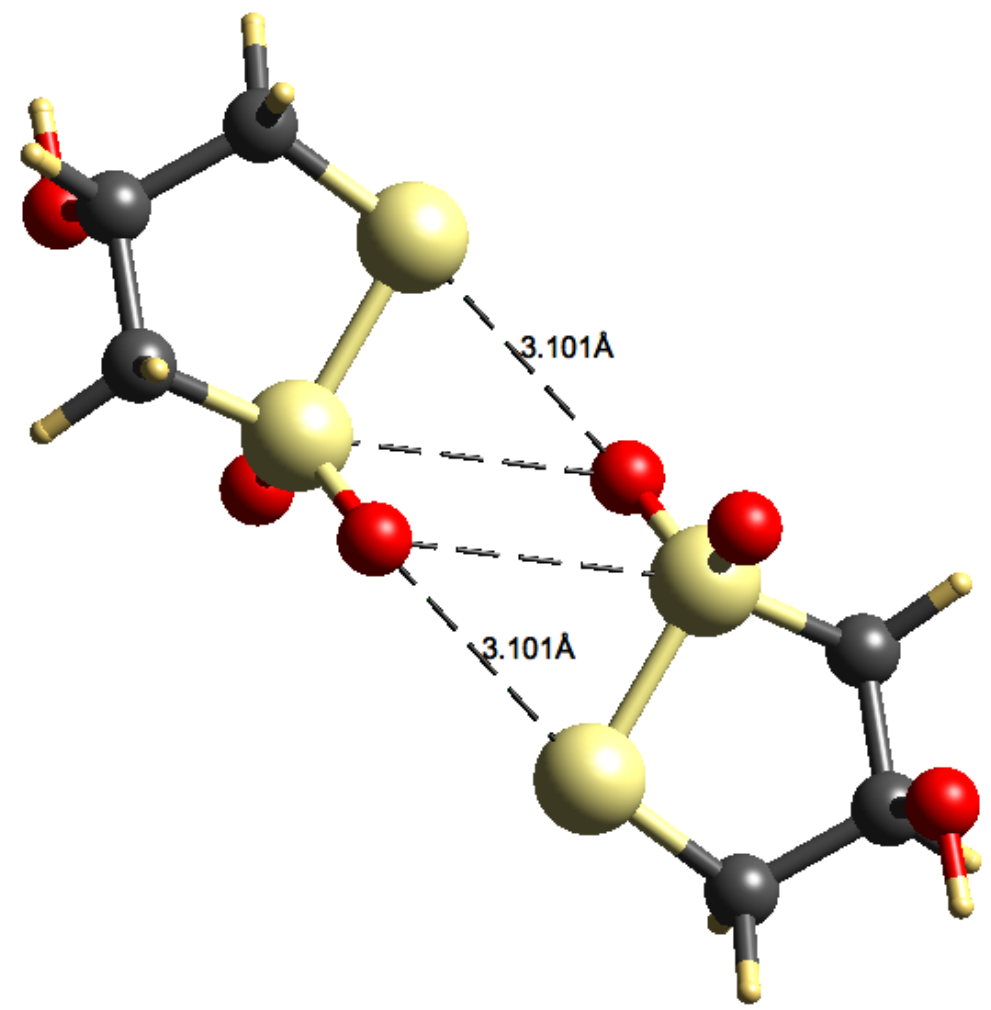

Figure S88: The molecular structure of $\mathrm{ADOFEF}^{m *}$.

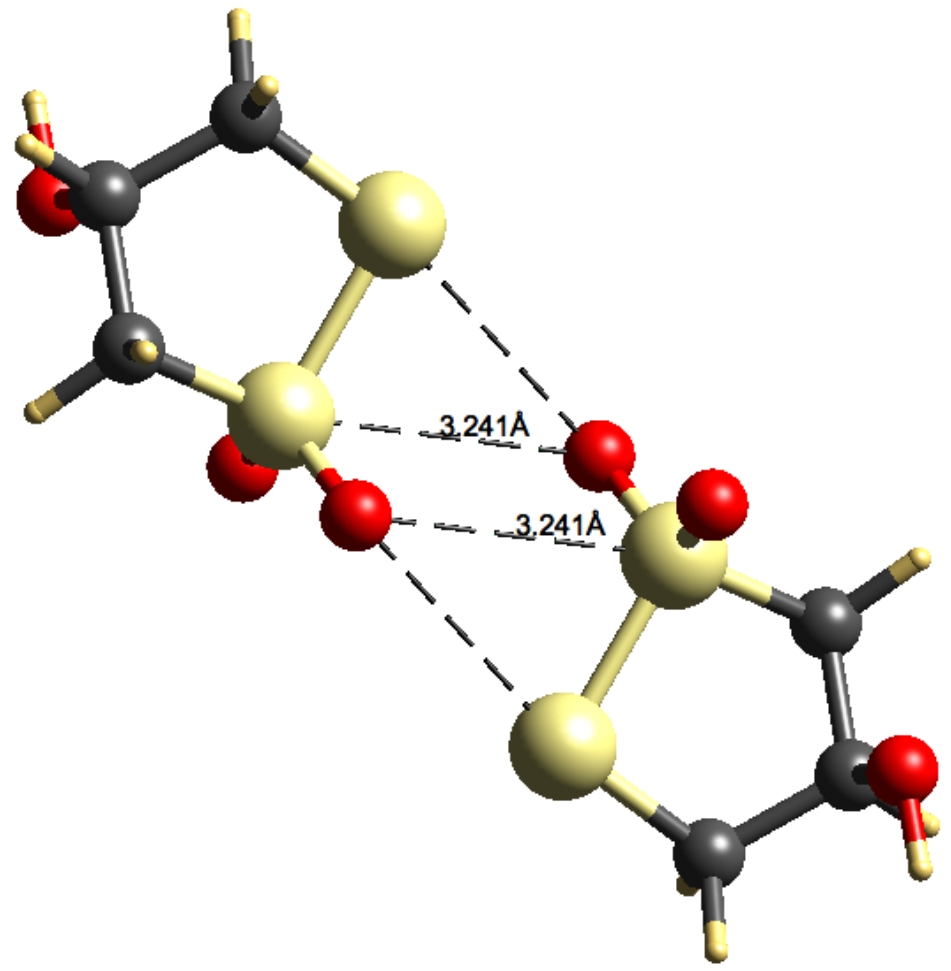

Figure S89: The molecular structure of $\mathrm{ADOFEF}^{m *}$. 


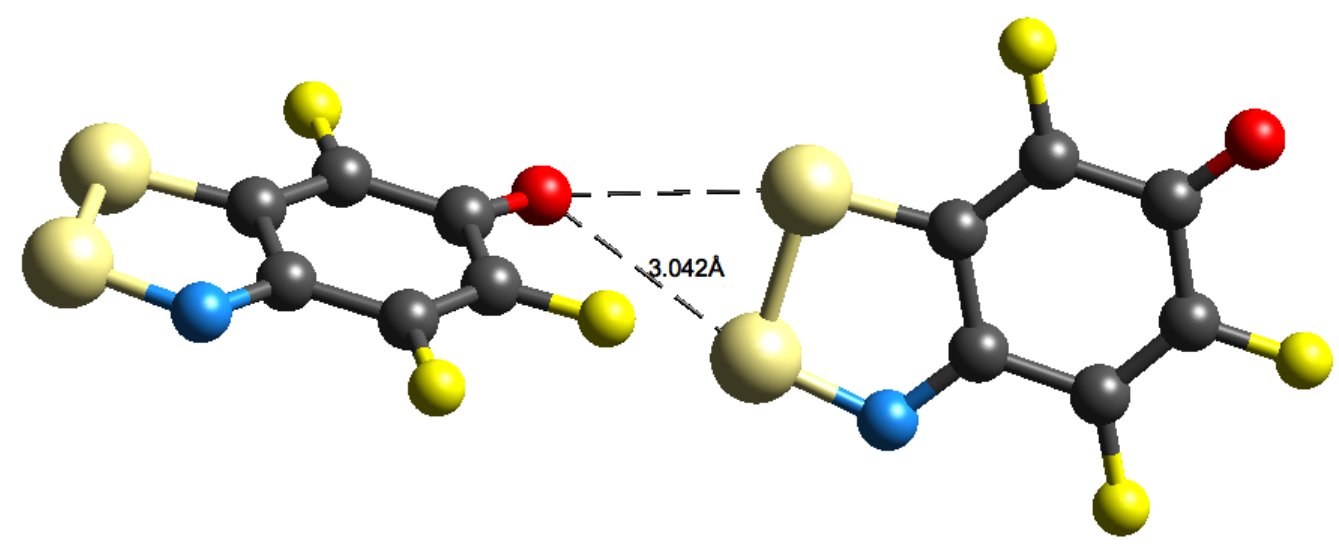

Figure S90: The molecular structure of MAVRAD ${ }^{m}$.

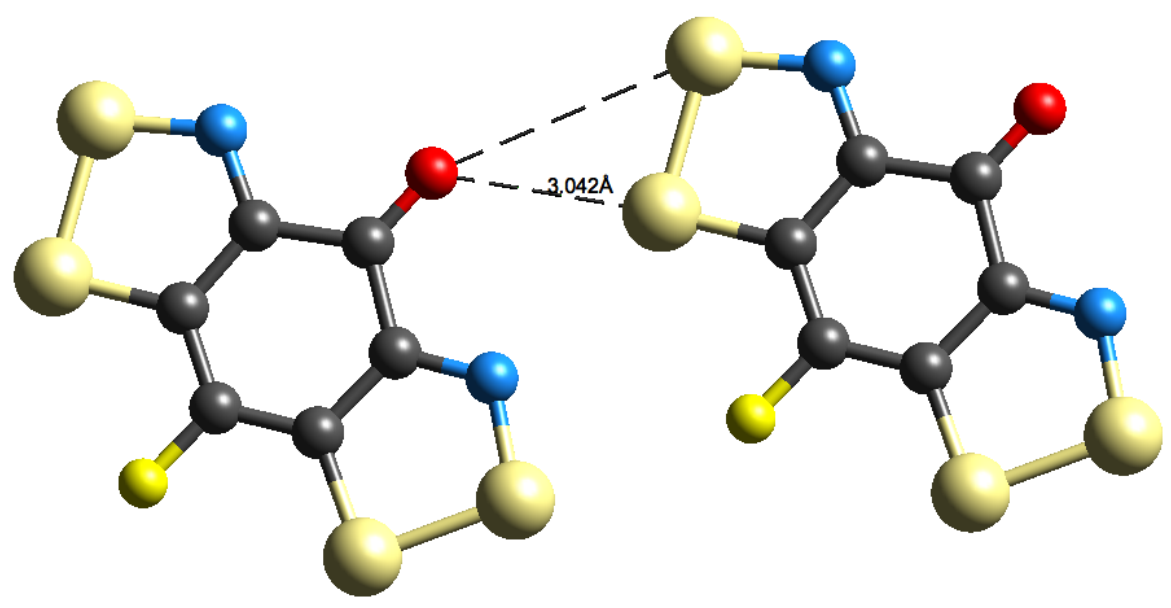

Figure S91: The molecular structure of $\mathrm{MEHNIY}^{m}$.

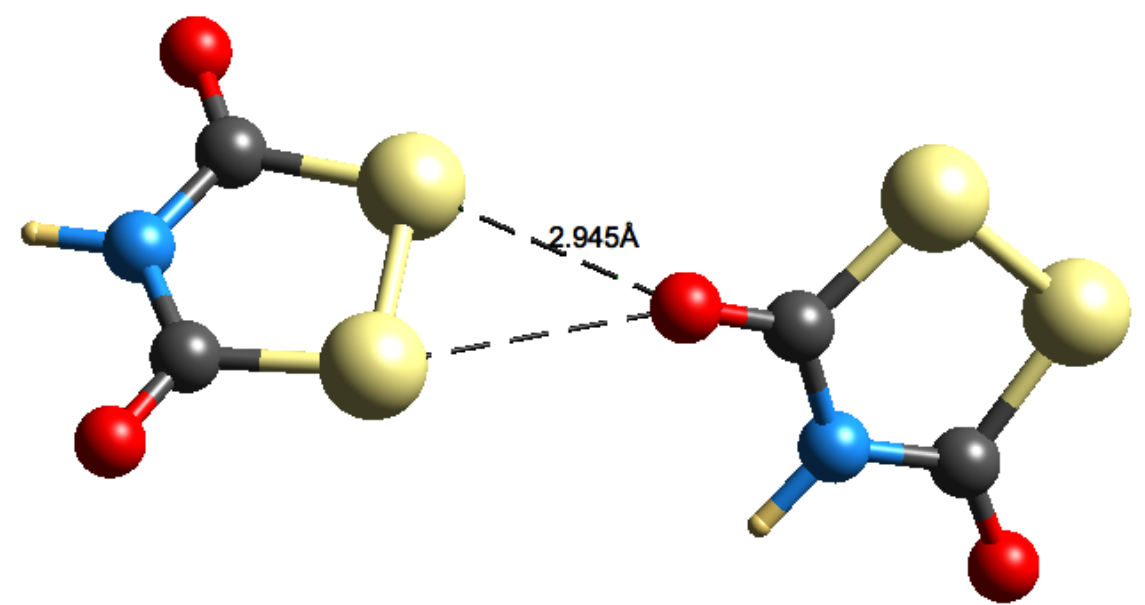

Figure S92: The molecular structure of NAHMUE ${ }^{m}$. 


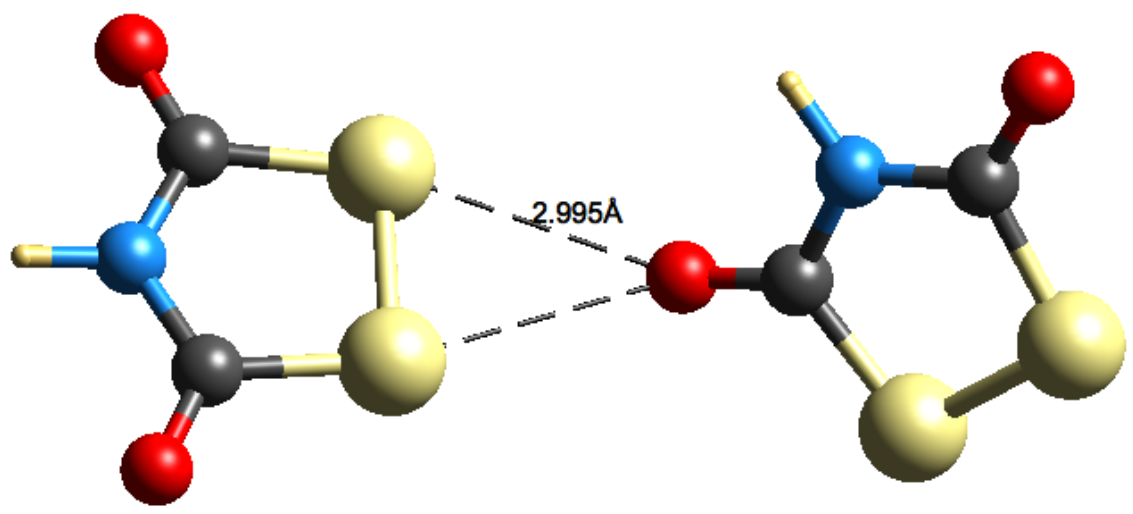

Figure S93: The molecular structure of NAHMUE ${ }^{m}$.

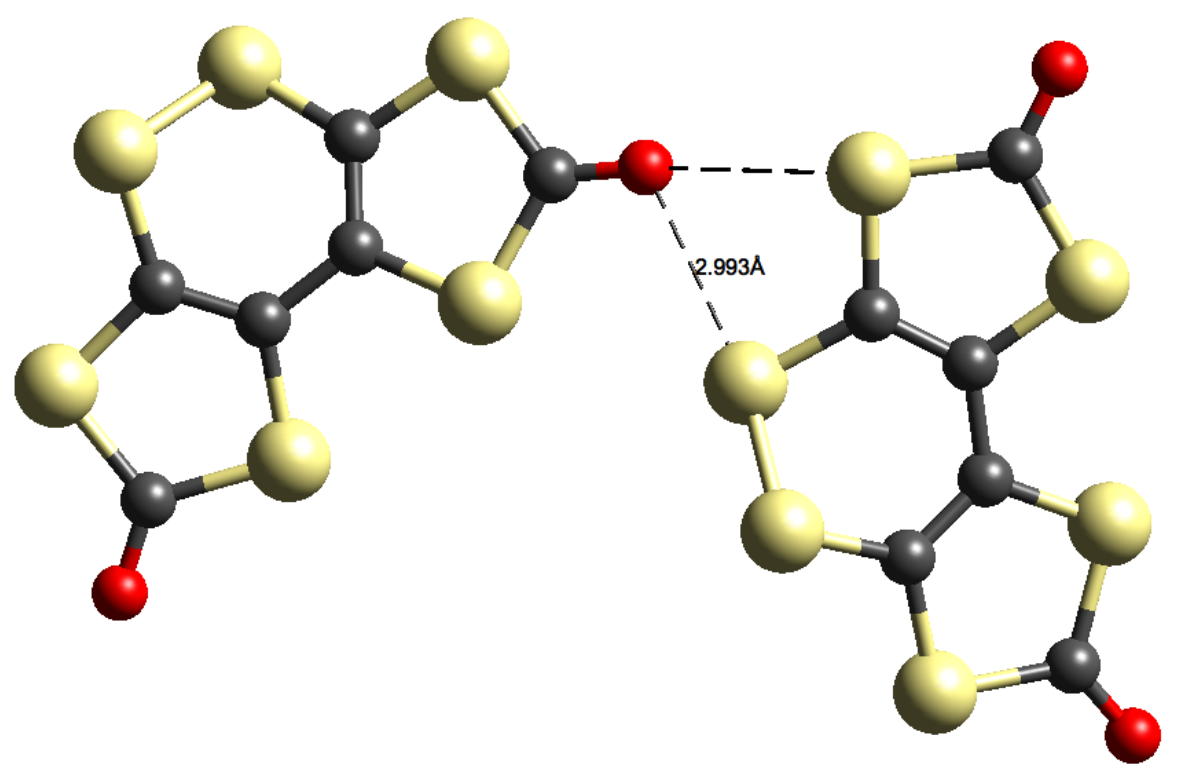

Figure S94: The molecular structure of PUDMUW ${ }^{m}$. 


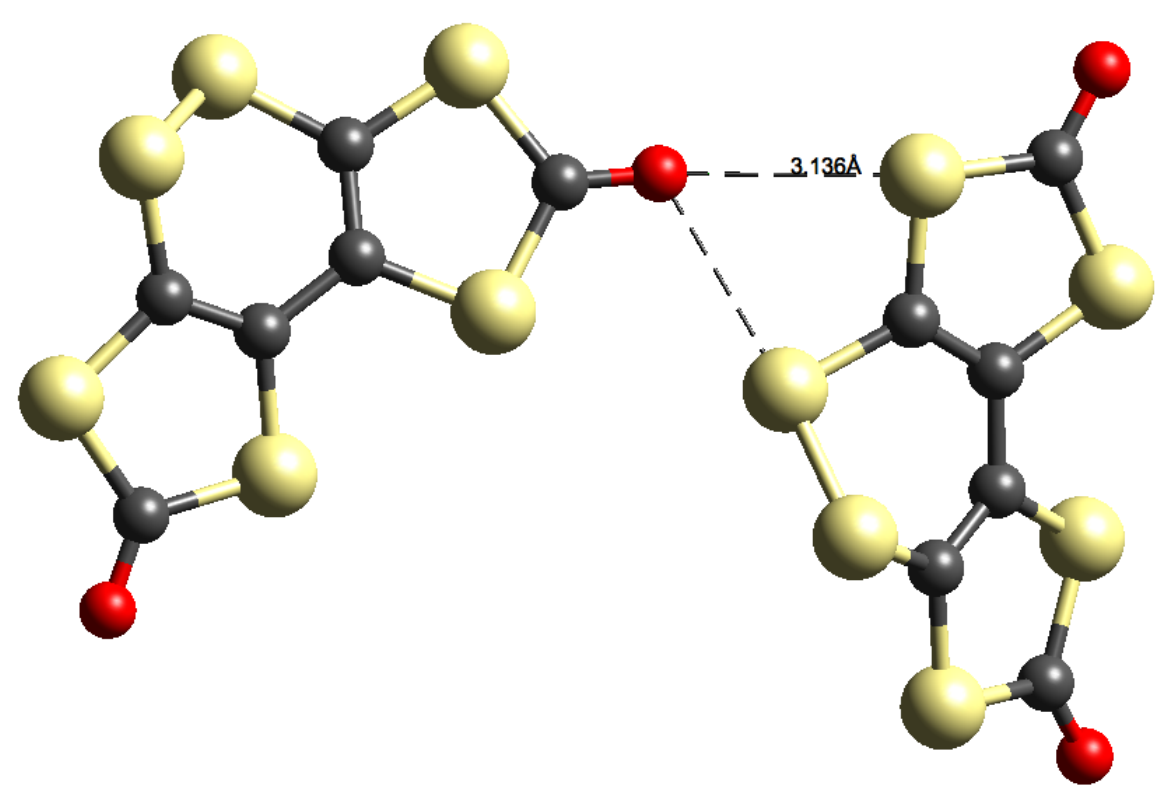

Figure S95: The molecular structure of PUDMUW ${ }^{m}$.

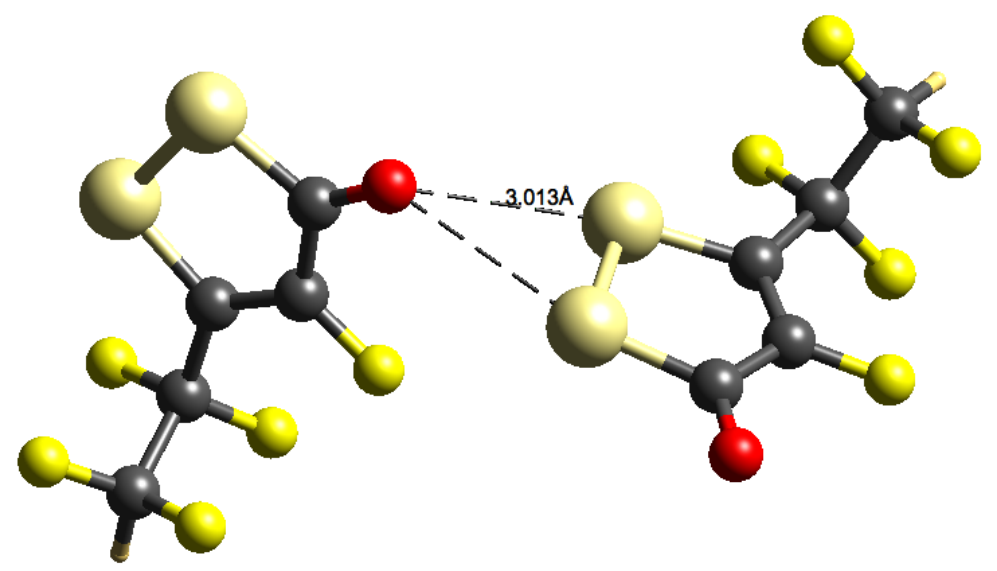

Figure S96: The molecular structure of QELQEE ${ }^{m}$.

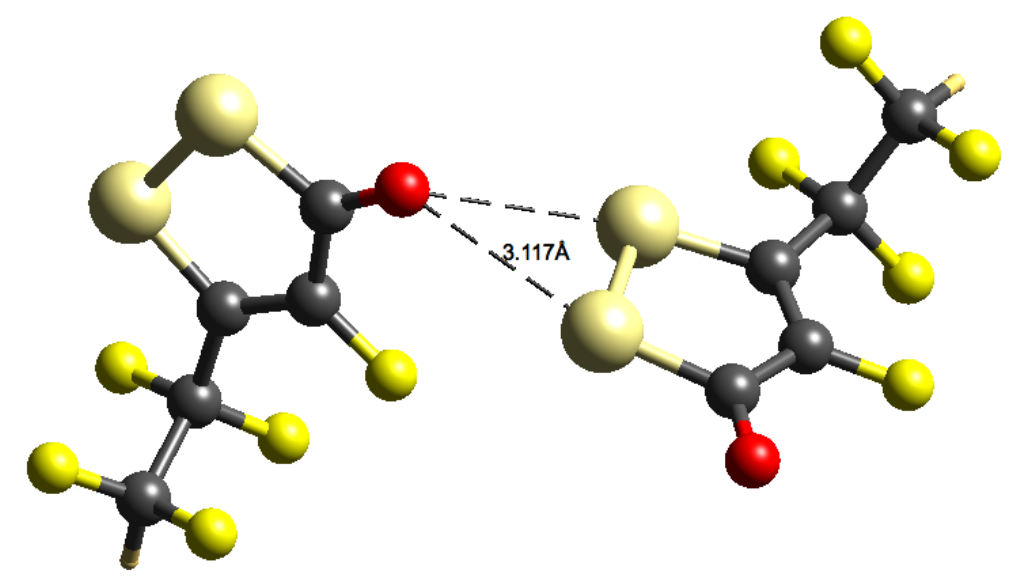

Figure S97: The molecular structure of $\mathrm{QELQEE}^{m}$. 


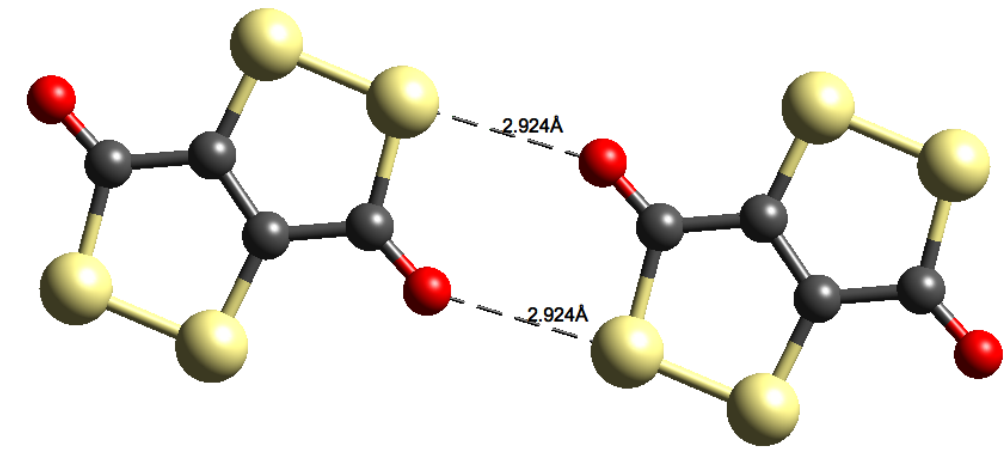

Figure S98: The molecular structure of ZAVHEJ ${ }^{m *}$. 
S8.3.3 Se $\cdots N$ interactions

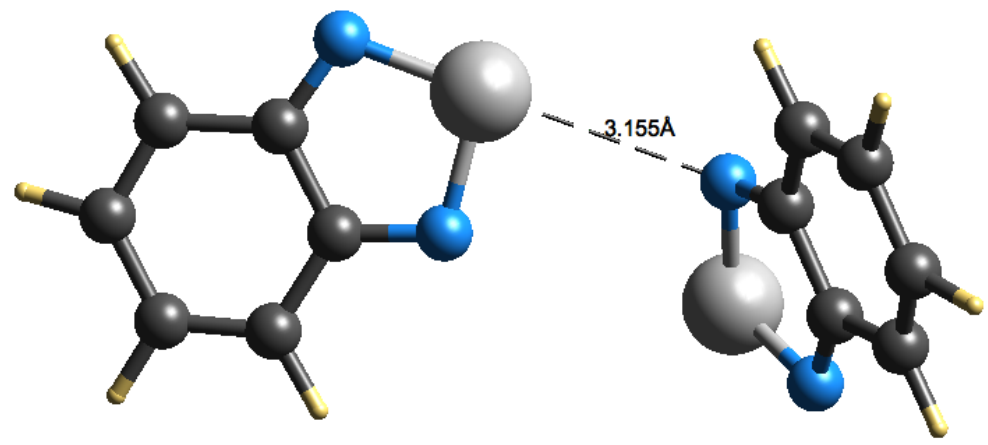

Figure S99: The molecular structure of BESEAZ01 ${ }^{s}$.

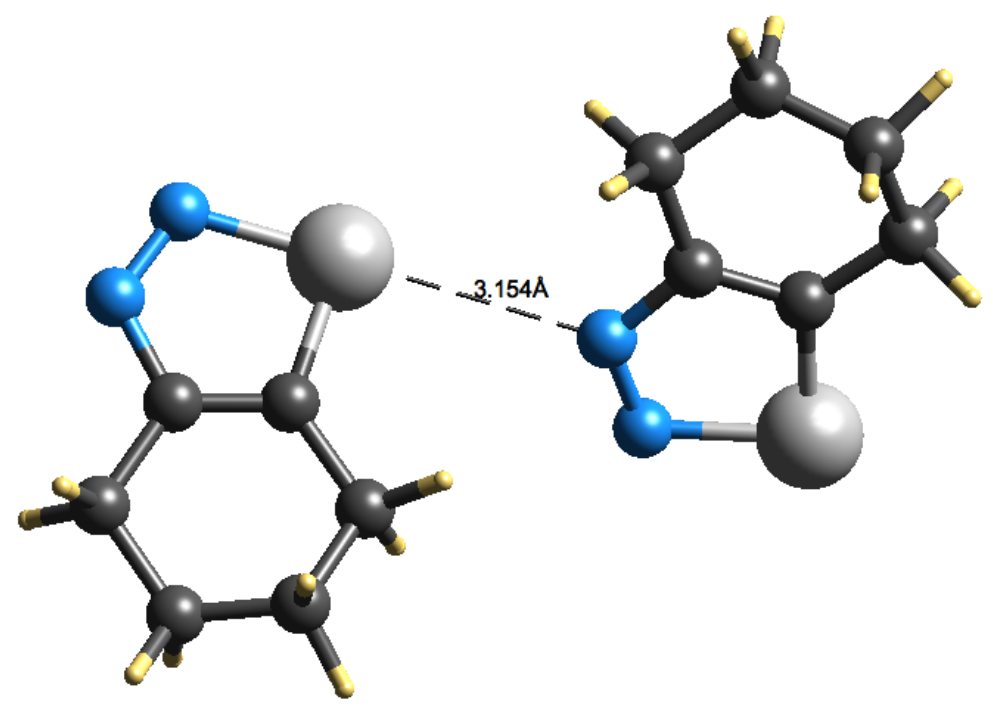

Figure S100: The molecular structure of FENFION ${ }^{s}$. 


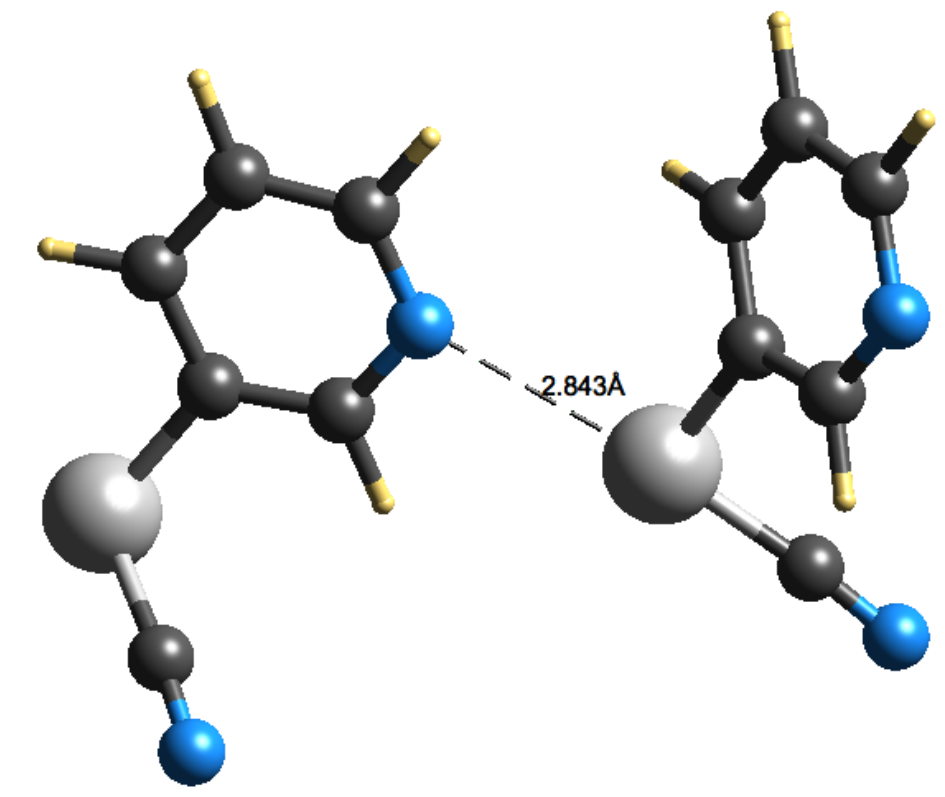

Figure S101: The molecular structure of WERYAT ${ }^{s}$.

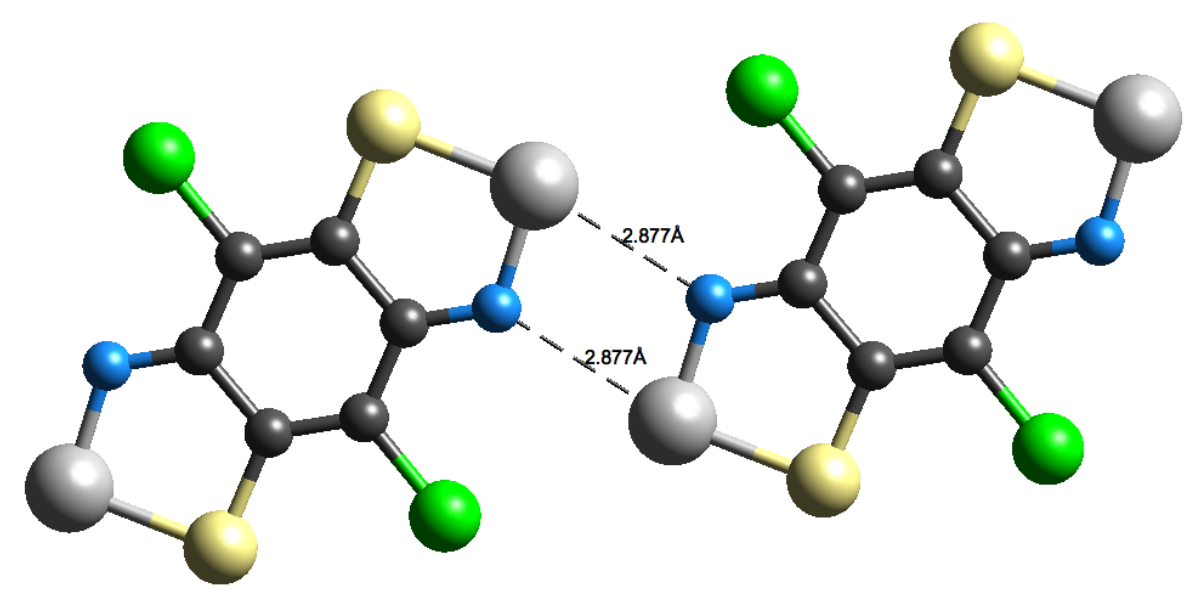

Figure S102: The molecular structure of NECZUQ ${ }^{m *}$. 


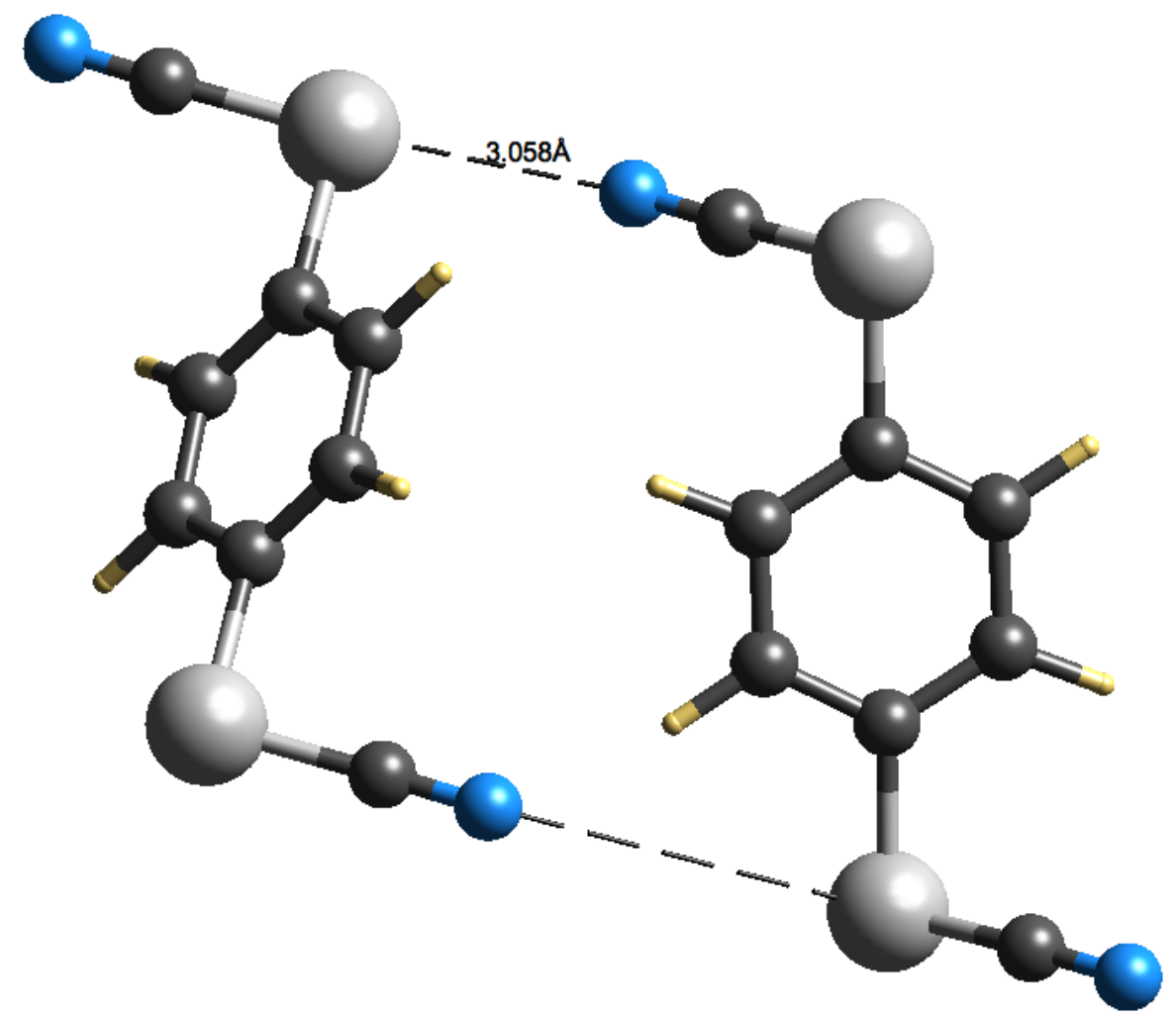

Figure S103: The molecular structure of $\mathrm{SECNBZ}^{m}$. 
S8.3.4 Se $\cdots O$ interactions

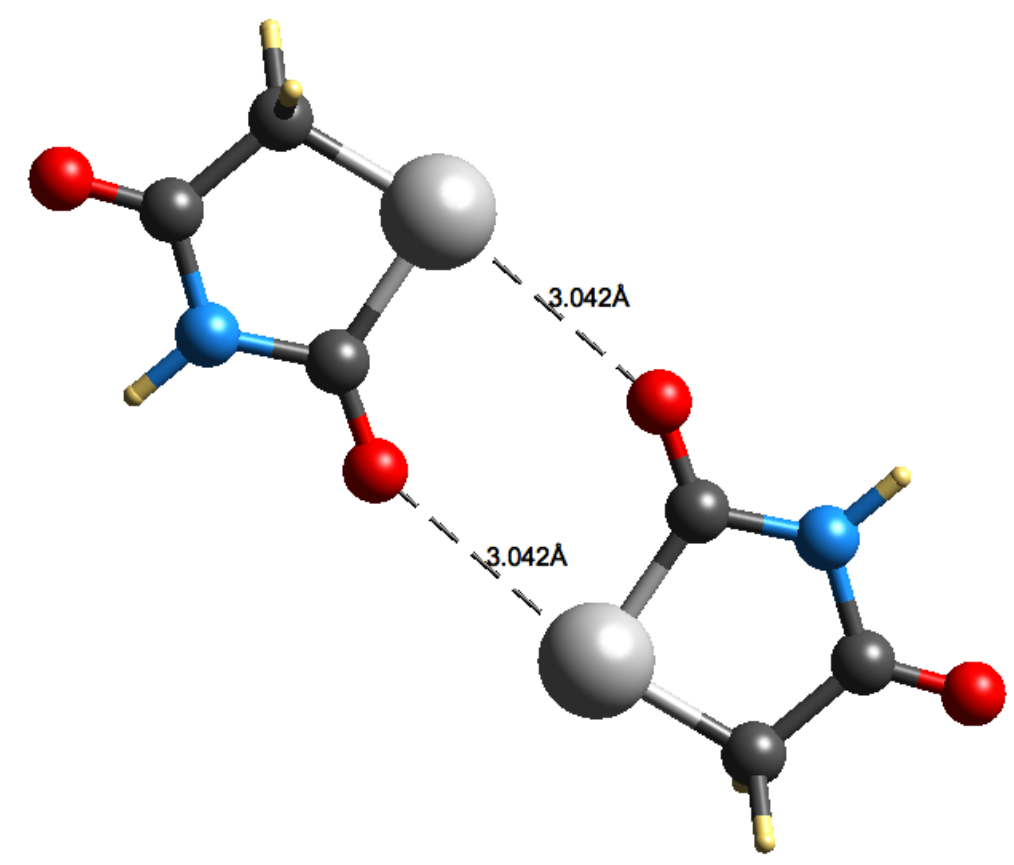

Figure S104: The molecular structure of BOJCOS ${ }^{m *}$.

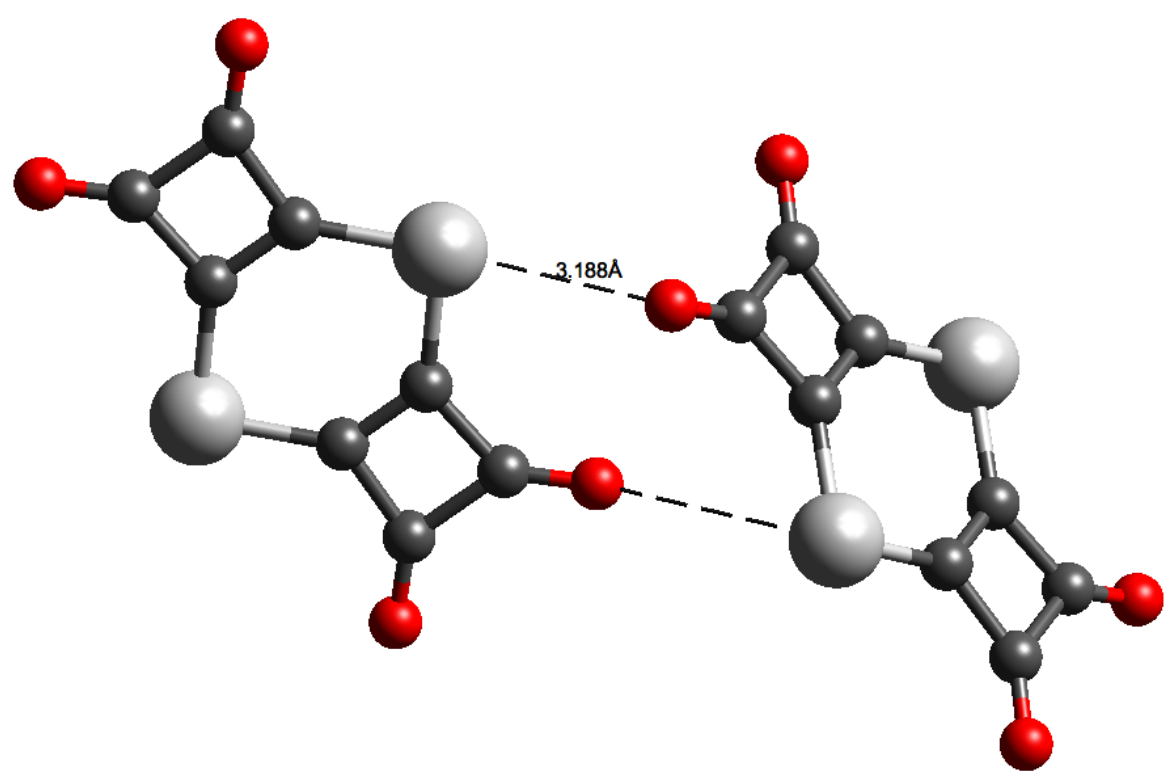

Figure S105: The molecular structure of LEDGAD ${ }^{m}$. 


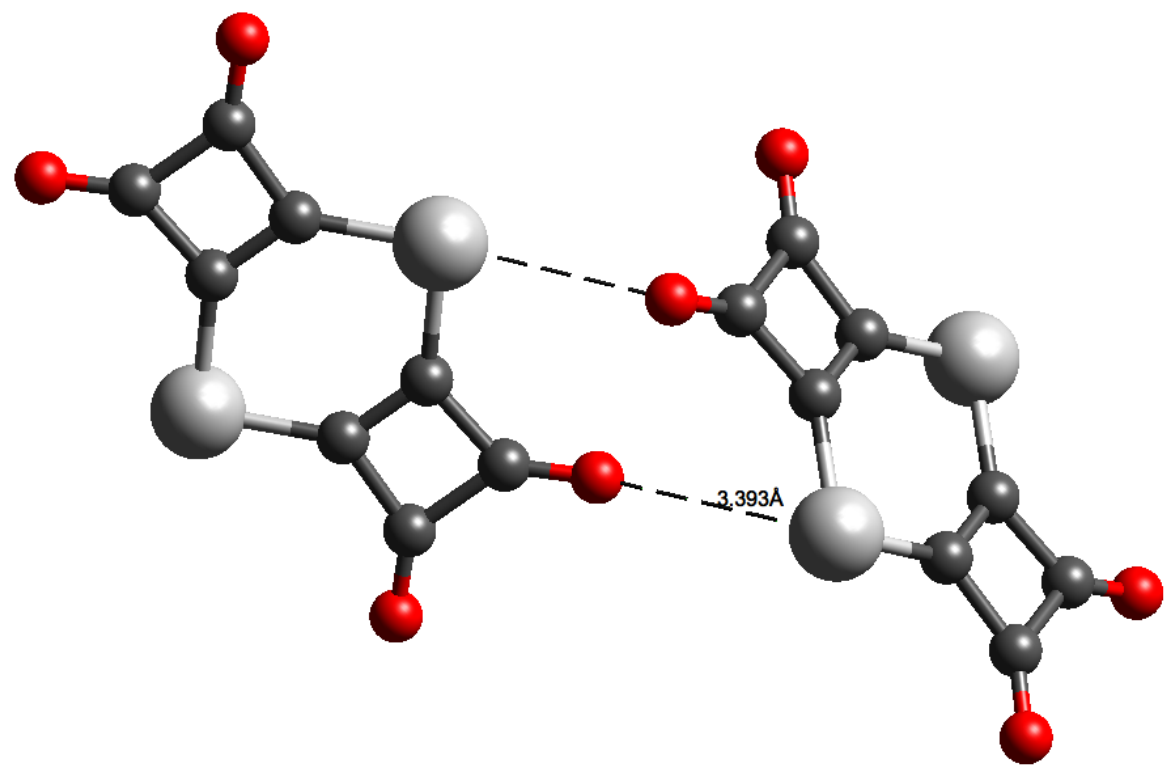

Figure S106: The molecular structure of $\mathrm{LEDGAD}^{m}$.

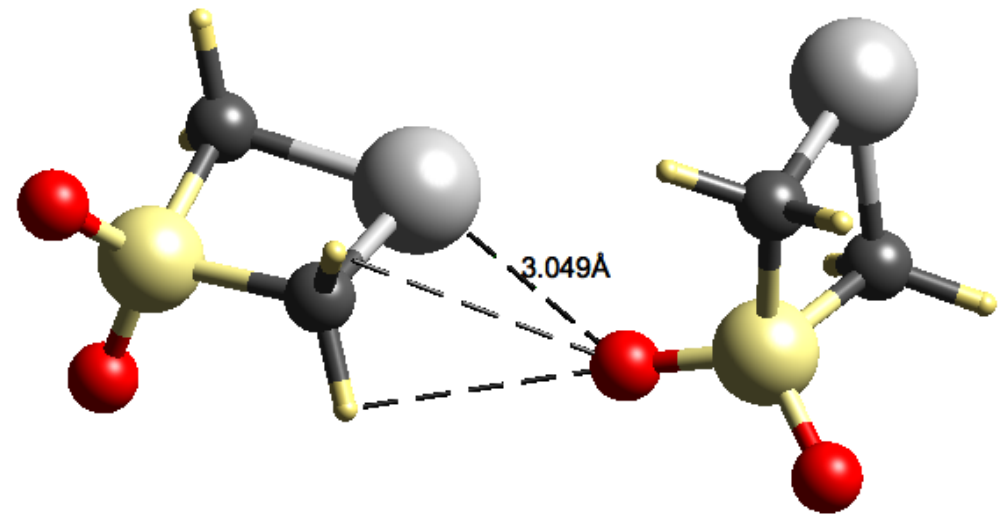

Figure S107: The molecular structure of LEVJOM ${ }^{m}$. 


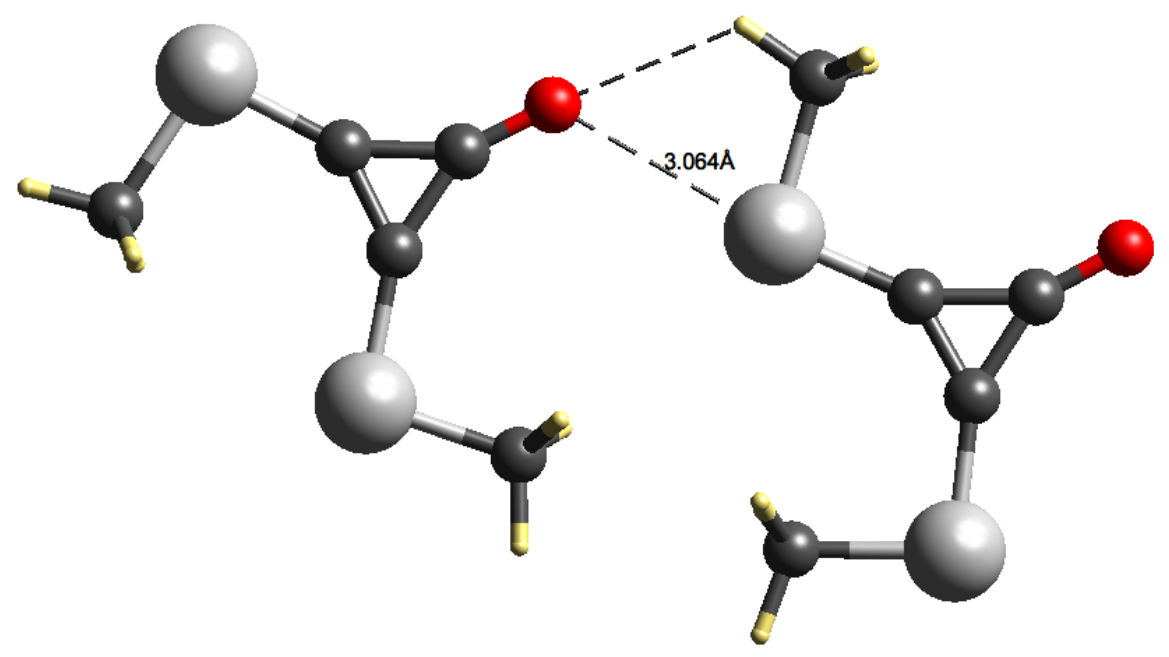

Figure S108: The molecular structure of $\mathrm{MUSCIM}^{m}$. 